\title{
BRIDGE NUMBER, HEEGAARD GENUS AND NON-INTEGRAL DEHN SURGERY
}

\author{
KENNETH L. BAKER, CAMERON GORDON, AND JOHN LUECKE
}

\begin{abstract}
We show there exists a linear function $w: \mathbb{N} \rightarrow \mathbb{N}$ with the following property. Let $K$ be a hyperbolic knot in a hyperbolic 3-manifold $M$ admitting a non-longitudinal $S^{3}$ surgery. If $K$ is put into thin position with respect to a strongly irreducible, genus $g$ Heegaard splitting of $M$, then $K$ intersects a thick level at most $2 w(g)$ times. Typically, this shows that the bridge number of $K$ with respect to this Heegaard splitting is at most $w(g)$, and the tunnel number of $K$ is at most $w(g)+g-1$.
\end{abstract}

\section{INTRODUCTION}

Let $M=K^{\prime}(p / q)$ be the manifold obtained by $p / q$-Dehn surgery on a knot $K^{\prime}$ in $S^{3}$. (Here $p / q \in \mathbb{Q}, q>0$, parametrizes the slope on the boundary of the exterior of $K^{\prime}$ that runs $p$ times meridionally and $q$ times longitudinally around $K^{\prime}$.) We denote the core of the attached solid torus in $K^{\prime}(p / q)$ by $K$. Thus $K$ is a knot in $M$ with a Dehn surgery that yields $S^{3}$, and it is natural to investigate what properties of $K$ this entails. In the present paper we are interested in the relationship between $K$ and the Heegaard splittings of $M$; more specifically, if $S$ is a Heegaard surface for $M$, what can we say about the bridge number $b r(K)=b r_{S}(K)$ of $K$ with respect to $S$ ? First note that if $S$ is a Heegaard surface for $X=S^{3}-N\left(K^{\prime}\right)$, the exterior of $K^{\prime}$, i.e., $S$ separates $X$ into a handlebody and a compression body, then $S$ becomes a Heegaard surface for $M$ such that $b r_{S}(K)=0$. In general not every Heegaard surface for $M$ arises in this way; for example the Heegaard genus of $M$ may be smaller than that of $X$. Nevertheless, it is shown in [27] (see also [23] and [26]) that, given $K^{\prime}$, for all but finitely many slopes $p / q$ we have $b r_{S}(K)=0$ for any Heegaard surface $S$ of $M$.

Recall that if $\gamma, \tau$ are two isotopy classes of simple closed curves on a 2-torus, then the distance between $\gamma$ and $\tau$, denoted $\Delta(\gamma, \tau)$, is defined to be the absolute value of the intersection number between $\gamma$ and $\tau$. Note that $q=\Delta(p / q, 1 / 0)$, the distance of $p / q$ from the meridian of $K^{\prime}$ on $\partial X$. Since the trivial Dehn surgery $K^{\prime}(1 / 0)=S^{3}$ represents the maximal possible degeneration of Heegaard genus, one would expect the Heegaard splittings of $K^{\prime}(p / q)$ to behave better as $q$ gets large.

Received by the editors February 1, 2012 and, in revised form, September 6, 2013.

2010 Mathematics Subject Classification. Primary 57M27.

In the course of this work the first author was partially supported by NSF Grant DMS-0239600, by the University of Miami 2011 Provost Research Award, and by a grant from the Simons Foundation (\#209184). The first author would like to thank the Department of Mathematics at the University of Texas at Austin for its hospitality during his visits. These visits were supported in part by NSF RTG Grant DMS-0636643.

The second author was partially supported by NSF Grant DMS-0906276. 
Indeed, it follows from [26] that if $K^{\prime}$ is hyperbolic and $S$ is a Heegaard surface of genus $g$ for $K^{\prime}(p / q)$, then $q \geq 18(g+1)$ implies $b r_{S}(K)=0$.

Here we consider the question whether there is an upper bound on $b r_{S}(K)$ that depends only on the genus of $S$ :

Question 1. Is there a function $w: \mathbb{N} \rightarrow \mathbb{N}$ such that if $K^{\prime}$ is a knot in $S^{3}$ and $S$ is a Heegaard surface of genus $g$ for $M=K^{\prime}(p / q)$, where $q>0$, then $b r_{S}(K) \leq w(g)$ ?

It turns out that the answer to Question 1 is "no" in general when $q=1$; see Remark 1.6 below. However, Corollary 1.1 below says that the answer is "yes" if $q \geq 2$, provided that $K^{\prime}$ is hyperbolic, $M$ is not a special kind of Seifert fibered space, and $p / q$ is not a boundary slope for $X$. Corollary 1.1 follows from our main result, Theorem 8.1, where we consider more generally the number of intersections of $K$ with a thick level surface in a Heegaard splitting of $M$ with respect to which $K$ is in thin position.

Theorem 8.1. There is a linear function $w: \mathbb{N} \rightarrow \mathbb{N}$ with the following property. Let $K^{\prime}$ be a hyperbolic knot in $S^{3}, M=K^{\prime}(p / q)$ where $q \geq 2$, and $K$ the core of the attached solid torus in $M$. Suppose $K$ is in thin position with respect to a genus $g$ Heegaard splitting of $M$ and let $S$ be a corresponding thick level surface. If $S$ is a strongly irreducible Heegaard surface for $M$, then either

(1) $|K \cap S| \leq 2 w(g)$; or

(2) $M$ is toroidal; or

(3) $M$ is a Seifert fibered space over the 2 -sphere with exactly three exceptional fibers, at least one of which has order 2 or 3 .

Furthermore, in cases (2) and (3) $M$ has a genus 2 Heegaard splitting with respect to which $K$ has bridge number 0 in case (2) and at most $w(2)$ in case (3).

In section 8 we show that $w(g)=10,581(g-1)+394$ works. The technical assumption in Theorem 8.1 that $S$ is strongly irreducible is likely unnecessary. It is only used in the proof of Lemma 5.12 .

If $X$ is a compact, orientable 3-manifold with torus boundary, let us say that a slope $r$ on $\partial X$ is a $(g, b)$-boundary slope for $X$ if there is a compact, connected, orientable essential surface $(F, \partial F) \subset(X, \partial X)$ with non-empty boundary and boundary slope $r$, such that $F$ has genus at most $g$ and at most $b$ boundary components. We say that $r$ is a $g$-boundary slope if it is a $(g, b)$-boundary slope for some $b$.

Corollary 1.1. There is a linear function $w: \mathbb{N} \rightarrow \mathbb{N}$ with the following property. Let $K^{\prime}$ be a hyperbolic knot in $S^{3}, M=K^{\prime}(p / q)$ where $q \geq 2$, and $K$ the core of the attached solid torus in $M$. Let $S$ be a genus $g$, strongly irreducible Heegaard surface in $M$. If $p / q$ is not a $(g, 2 w(g)-2)$-boundary slope for the exterior of $K^{\prime}$, then either $\operatorname{br}_{S}(K) \leq w(g)$, or conclusion (3) of Theorem 8.1 holds.

Proof. For terminology on thin presentations, see section 2.1. Let $w$ be the function of Theorem 8.1 Assume $K^{\prime}(p / q)$ has a genus $g$ splitting which is strongly irreducible, and that $p / q$ is not a $(g, 2 w(g)-2)$-boundary slope. In particular, $M$ cannot be toroidal since, otherwise, by [17] $p / q$ is a $(1,2)$-boundary slope (and $g>1$ ). Put $K$ in thin position with respect to this splitting and let $S$ be a thick level surface. Theorem 8.1 says that either $M$ is the Seifert fibered space of its 
conclusion (3) and that $K$ has bridge number at most $w(2)$ with respect to a genus 2 splitting of $M$, or $|K \cap S| \leq 2 w(g)$. This thin presentation of $K$ must be a bridge presentation of $K$, otherwise there will be a thin level surface in the thin presentation which intersects $K$ fewer times than does $S$, and Lemma 2.2 would contradict that $p / q$ is not a $(g, 2 w(g)-2)$-boundary slope. Thus $K$ is bridge with respect to $S$ and $b r_{S}(K) \leq w(g)$.

Remark 1.2. By [18, at most finitely many slopes of the exterior of $K^{\prime}$ are boundary slopes. Note that if $M$ is non-Haken, then $p / q$ cannot be a boundary slope, by Theorem 2.0.3 of [7] ( $q \geq 2$ and $M$ is irreducible by [14]). If $M$ is a Seifert fibered space over the 2 -sphere with at most three exceptional fibers, then, since $p \neq 0, M$ is non-Haken.

Remark 1.3. It is conjectured that a Seifert fibered space can never be obtained by non-integral Dehn surgery on a hyperbolic knot in $S^{3}$ - that is, that (3) of Theorem 8.1 never occurs. Theorem 2.4 of [3] implies that if $M$ is Seifert fibered over the 2 -sphere with three exceptional fibers and $q>2$, then $K$ has bridge number at most 1 with respect to some genus 2 Heegaard splitting of $M$, though possibly a 1 -sided splitting (which are not considered in this paper). Note that since a 1-sided splitting corresponds to a non-orientable surface, if $p$ is odd, then $K^{\prime}(p / q)$ does not have a 1-sided splitting. Thus when $q>2$ and $p$ is odd, Theorem 2.4 of [3] sharpens conclusion (3) above.

Remark 1.4. If $M$ is toroidal, then by [17] ( $q=2$ and) $K^{\prime}$ belongs to the family of knots described by Eudave-Muñoz in [8]. In [9] Eudave-Muñoz shows that these knots have tunnel number 1 , and hence $b r(K)=0$ with respect to a minimal genus (genus 2) splitting of $M$.

Remark 1.5. We conjecture that the hypotheses that $K^{\prime}$ is hyperbolic in Theorem 8.1 and Corollary 1.1, and that $p / q$ is not a $(g, 2 w(g)-2)$-boundary slope in Corollary 1.1, are redundant.

Remark 1.6. When $q=1$ the answer to Question 1 is "no" in general. In 30] Teragaito constructs infinitely many hyperbolic knots $K_{n}^{\prime}$ in $S^{3}$ such that 4-surgery on $K_{n}^{\prime}$ gives the same 3 -manifold $M$ for all $n$, where $M$ is a Seifert fibered space of type $S^{2}(2,6,7)$. In particular $M$ has Heegaard genus 2 . Let $K_{n}$ be the core of the attached solid torus in $K_{n}^{\prime}(4)=M$, and define $b\left(K_{n}\right)=\min \left\{b r_{S}\left(K_{n}\right): S\right.$ a genus 2 Heegaard surface for $M\}$. It is shown in 2 that $b\left(K_{n}\right)$ is unbounded.

Remark 1.7. For small values of $g$ the bridge number $\operatorname{br}(K)$ is either known or conjectured to be very small. For example, the impossibility of getting $S^{3}$ by nontrivial Dehn surgery on a non-trivial knot [15] can be expressed as saying that if $g=0$ and $q>0$, then $\operatorname{br}(K)=0$. When $g=1, K^{\prime}(p / q)$ is a lens space, and here the Cyclic Surgery Theorem [7] says that if $q>1$, then $K^{\prime}$ is a torus knot, which is easily seen to imply $\operatorname{br}(K)=0$, while if $q=1$ and $K^{\prime}$ is hyperbolic, the Berge Conjecture [4] is equivalent to the assertion that $\operatorname{br}(K)=1$. In another paper ( $[3$, Theorem 2.4]), we consider the case $g=2$ and show that if $q>2$, then, generically, $\operatorname{br}(K) \leq 1$ (with respect to some genus 2 splitting, possibly 1-sided).

Remark 1.8. Strongly irreducible Heegaard surfaces and closed incompressible surfaces may exhibit similar behavior. With this theme in mind, in the Appendix we adapt the proof of Theorem 8.1 to bound the intersection number of $K$ with an 
incompressible surface rather than a thick Heegaard surface in terms of the genus of the surface:

Theorem 9.1. There is a linear function $w_{I}: \mathbb{N} \rightarrow \mathbb{N}$ with the following property. Let $K^{\prime}$ be a hyperbolic knot in $S^{3}, M=K^{\prime}(p / q)$ where $q \geq 2$, and $K$ the core of the attached solid torus in $M$. Let $S$ be an orientable, incompressible surface in $M$ of genus $g$. Then $K$ can be isotoped to intersect $S$ at most $w_{I}(g)$ times.

Remark 1.9. Osoinach shows that for integral surgeries the above does not hold. In [25], he gives examples of infinitely many different knots in the 3-sphere on which 0 -surgery gives the same manifold. This manifold has an essential torus, and in 24] he shows that the set of minimal intersection numbers in $M$ of this torus with the corresponding cores of the attached solid tori must be infinite. For a sharpening of Osoinach's result see 31.

Our results give information on the relationship between the Heegaard genus of $M$ and that of $X$, the exterior of $K^{\prime}$. Recall that a Heegaard splitting of $X$ is a decomposition $X=V \bigcup_{S} W$, where $V$ is a handlebody with $\partial V=S$ and $W$ is a compression body with $\partial W=S \sqcup \partial X$. The Heegaard genus $g(X)$ of $X$ is the minimal genus of $S$ over all such decompositions. In this context one often talks about the tunnel number $t\left(K^{\prime}\right)$ of $K^{\prime}$, the minimum number of arcs ("tunnels") that need to be attached to $K^{\prime}$ so that the complement of an open regular neighborhood of the resulting 1-complex is a handlebody. It is easy to see that $g(X)=t\left(K^{\prime}\right)+1$. For any slope $p / q, V \bigcup_{S} W(p / q)$ is a Heegaard splitting of $M=K^{\prime}(p / q)$; in particular, $g(M) \leq g(X)$. The question arises as to what extent the Heegaard genus can decrease under Dehn filling, i.e., how bad the inequality $g(M) \leq g(X)=t\left(K^{\prime}\right)+1$ can be. Since it is easy to see that if $K$ has bridge number $b r(K)$ with respect to a genus $g$ splitting of $M$, then $t(K)\left(=t\left(K^{\prime}\right)\right) \leq g+b r(K)-1$, Corollary 1.1 gives a linear bound on the extent to which the Heegaard genus can decrease under a non-integral Dehn surgery.

Corollary 1.10. There is a linear function $w_{T N}: \mathbb{N} \rightarrow \mathbb{N}$ with the following property. Let $K^{\prime}$ be a hyperbolic knot in $S^{3}$ and $M=K(p / q)$. If $M$ has a strongly irreducible genus $g$ Heegaard surface, $p / q$ is not a $\left(g, 2\left(w_{T N}(g)-g\right)\right)$-boundary slope for the exterior of $K^{\prime}$, and $q \geq 2$, then the tunnel number of $K^{\prime}$ is at most $w_{T N}(g)$.

Remark 1.11. In Corollaries 1.1 and 1.10, the assumption that the genus $g$ Heegaard surface is strongly irreducible is added for clarity but is unnecessary once the assumption is made that $p / q$ is not a $(g, 2 w(g)-2)$-boundary slope. Suppose that $S$ is a weakly reducible Heegaard surface for $M$ and $p / q$ is not a $(g, 2 w(g)-2)-$ boundary slope for $K^{\prime}$. We may assume that $S$ is irreducible. Untelescope the splitting corresponding to $S$ (see section 3 of [29]) into a collection of strongly irreducible splittings (by compression bodies, each of genus smaller than $g$ ) separated by an incompressible surface, $F$, in $M$. Note that the surface $F$ is not necessarily connected but each component of $F$ has genus less than $g$. Since $p / q$ is not a $(g, 2 w(g)-2)$-boundary slope, Theorem 9.1 says that $K$ can be isotoped to be disjoint from $F$ (as stated in the proof of Theorem 9.1 one can take $w_{I}(g)=2 w(g)$ ). Let $M^{\prime}$ be the component of $M-\operatorname{Nbhd}(F)$ containing $K$, and let $S^{\prime}$ be the genus $g^{\prime}$ Heegaard surface of the (compression-body) splitting of $M^{\prime}$ coming from the untelescoping. Putting $K$ in thin position with respect to this splitting (e.g. section 2.3 of [2]) and applying the same arguments as for Theorem 8.1] and Corollary 1.1, 
using $S^{\prime}$ in place of $S$ and noting that $F$ is incompressible in $M$, we conclude that $K$ has a bridge presentation with respect to $S^{\prime}\left(p / q\right.$ is not a $\left(g^{\prime}, 2 w\left(g^{\prime}\right)-2\right)$-boundary slope) with bridge number at most $w\left(g^{\prime}\right)<w(g)$. By the amalgamation procedure (section 3, 29]) we get a bridge presentation, with the same bridge number, with respect to the splitting of $M$ given by $S$.

We conjecture that there is always such a universal bound on the tunnel number of a knot in terms of the Heegaard genus of any of its (non-trivial Dehn surgeries):

Conjecture 1.12. There is a function $w_{T N}: \mathbb{N} \rightarrow \mathbb{N}$ such that if $K^{\prime}$ is a knot in $S^{3}$ and $M=K(p / q)$ is a non-trivial Dehn surgery on $K^{\prime}$ with Heegaard genus $g$, then the tunnel number of $K^{\prime}$ is at most $w_{T N}(g)$.

Information on the question of degeneration of the Heegaard genus under Dehn filling in provided by Rieck and Sedgwick in 27] and 28. As mentioned above, it is shown in [27] that for all but finitely many slopes $p / q, \operatorname{br}(K)=0$ with respect to any Heegaard surface $S$ of $M$. Taking $S$ to have minimal genus, it is easy to see that $\operatorname{br}(K)=0$ implies that either $g(M)=g(X)=t\left(K^{\prime}\right)+1$ or $g(M)=$ $g(X)-1=t\left(K^{\prime}\right)$. See [26] for details. By [28], the second possibility can happen for only a finite number of lines of slopes (where a line is a set of slopes $r$ such that $\Delta\left(r, r_{0}\right)=1$ for some fixed slope $\left.r_{0}\right)$.

Regarding Corollary 1.10 and Conjecture 1.12, in fact we know of no examples where the Heegaard genus of $K^{\prime}(p / q)(q>0)$ is less than $t\left(K^{\prime}\right)$. So we ask

Question 2. Is $t\left(K^{\prime}\right) \leq g\left(K^{\prime}(p / q)\right)$ for all $q>0$ ?

For example, the answer to Question 2 is known to be "yes" when

- $g\left(K^{\prime}(p / q)\right)=0([15])$,

- $g\left(K^{\prime}(p / q)\right)=1$ and $q>1([7])$,

- $g\left(K^{\prime}(p / q)\right)=2, q>2$, and $K^{\prime}(p / q)$ does not contain an incompressible surface of genus $2(\underline{3}])$.

Finally, the bound on the bridge number in Corollary 1.1 allows us to use a result of Tomova 33. to get a statement about the distance of splittings of exteriors of knots with genus $g$ Dehn surgeries. If $S$ is a Heegaard surface for some 3-manifold, we denote by $d(S)$ the distance of the corresponding splitting; see [19].

Corollary 1.13. There is a linear function $w_{H D}: \mathbb{N} \rightarrow \mathbb{N}$ with the following property. Let $K^{\prime}$ be a hyperbolic knot in $S^{3}$ whose exterior has a genus $g$ Heegaard surface $S$ with $d(S)>w_{H D}(g)$. If $p / q$ is not a $\left(g, w_{H D}(g)-2 g-2\right)$-boundary slope for the exterior of $K^{\prime}$ and $q \geq 2$, then $g\left(K^{\prime}(p / q)\right)=g$.

Thus the distance of a splitting of a knot exterior is putting a limit on the degeneration of the Heegaard genus under Dehn filling. For instance, this applies to the examples of 21. Recall that a knot has only finitely many boundary slopes (18]). By [21, for any $g \geq 2$, there are knots $K^{\prime}$ in $S^{3}$ whose exteriors have genus $g$ Heegaard splittings $S$ with $d(S)>w_{H D}(g)$, in fact with $d(S)$ arbitrarily large (such knots are necessarily hyperbolic). (The case $g=2$ was first done in [20].) Corollary 1.13 says that for such a knot $K^{\prime}$, if $q \geq 2$ and $p / q$ is not a $\left(g, w_{H D}(g)-2 g-2\right)$-boundary slope, then $K^{\prime}(p / q)$ has Heegaard genus $g$. For results in a similar vein see [5].

Proof of Corollary 1.13. Let $K^{\prime}, p / q, S$ be as in the hypothesis. Set $w_{H D}(g)=$ $2 g+2 w(g)$. Assume for contradiction that $g\left(K^{\prime}(p / q)\right)=g^{\prime}<g$. By Corollary [1.1, 
the bridge number of $K$ with respect to some genus $g^{\prime}$ Heegaard surface $\widehat{F}$ of $K^{\prime}(p / q)$ is at most $w\left(g^{\prime}\right)$. Thus $K$ can be put in bridge position with respect to $\widehat{F}$ so that $2-\chi(\widehat{F}-K)=2-\left(2-2 g^{\prime}-2 w\left(g^{\prime}\right)\right) \leq 2 g+2 w(g)=w_{H D}(g)$. Then $d(S)>2-\chi(\widehat{F}-K)$ by assumption, and the main result of 33. implies that, in $K^{\prime}(p / q), \widehat{F}$ is isotopic to a stabilization of $S$. But $\widehat{F}$ has smaller genus than $S$.

Throughout this article $M$ will be the manifold $K^{\prime}(p / q)$ obtained by $p / q$-Dehn surgery on a hyperbolic knot $K^{\prime}$ in $S^{3}$, with $q \geq 2$. It follows that

(1) $M$ is irreducible [14;

(2) $M$ is not a lens space (or $S^{3}$ ) [7];

(3) $M$ does not contain a Klein bottle [16, 6].

Note that (1) and (2) together imply that $M$ does not contain a projective plane. We also assume (see Remark 1.4)

(4) $M$ is atoroidal.

1.1. Overview of the proof of Theorem 8.1. The proof of Theorem8.1 occupies the remainder of this article and culminates in section 8 . We give an overview, taking the notation from Theorem 8.1

We begin in section 2 by briefly reviewing and setting up notation for the notions of thin position and fat vertexed graphs of intersection between (punctured) Heegaard spheres of $S^{3}$ and Heegaard surfaces of $M$. In particular, $Q$ and $F$ are surfaces in the exterior of $K^{\prime}$ coming from a Heegaard sphere, $\widehat{Q}$, in $S^{3}$ and the given thick level Heegaard surface, $\widehat{F}=S$, in $M . G_{Q}, G_{F}$ are the graphs of intersection between $Q, F$. Let $t=|K \cap \widehat{F}|=|K \cap S|$ be the number of components of $\partial F$. Then $t$ is also the number of vertices of $G_{F}$ and consquently the number of different labels on the vertices of $G_{Q}$. Our goal is to bound $t$ by some linear function of $g$.

Lemma 2.5 shows the existence of a special subgraph, $\Lambda$, of $G_{Q}$ called a great $g$-web. For each label $x$ of this graph, $\Lambda_{x}$ is the subgraph of $\Lambda$ consisting of those edges with label $x$. We consider the set $\mathcal{L}$ of all labels, $x$, for which $\Lambda_{x}$ has a bigon or trigon face. In Proposition 2.9 we make a key estimate showing that up to an additive linear function in $g,|\mathcal{L}| \geq(3 / 4) t$.

A bigon or trigon of $\Lambda_{x}$ gives rise to a bigon or trigon subgraph in $\Lambda$. We classify these subgraphs as either extended Scharlemann cycles or as trigons of Type I, II, or III. The extended Scharlemann cycles give rise to long Möbius bands and long twisted $\theta$-bands embedded in $M$ which intersect $\widehat{F}$ in essential curves (or essential $\theta$-curves). We choose a minimal collection, $\Sigma$, of extended Scharlemann cycles so that each label of $\mathcal{L}$ that corresponds to an extended Scharlemann cycle appears as a label in some element of $\Sigma$. Let $L(\Sigma)$ be the set of all labels appearing in the extended Scharlemann cycles of $\Sigma$. Those labels of $\mathcal{L}$ that do not appear in $L(\Sigma)$ then correspond to trigons of Type I, II, or III. Section [6 analyzes trigons of Type I or II and culminates in Theorem 6.24, which bounds, in terms of a linear function in $g$, those labels appearing in trigons of Type I or II but not in $L(\Sigma)$ (the collection of these labels is called $\left.\mathcal{L}_{\mathrm{II}}\right)$. Section 7 shows that those labels appearing in Type III trigons but not in $L(\Sigma)$, up to a linear function in $g$ (Lemma 7.14), correspond to vertices in trivial curves on $\widehat{F}$ called simple gnarls. The collection of such labels is called $\mathfrak{G}$. That is, up to an added linear function in $g$, we have that $|\mathcal{L}|$ is $|L(\Sigma)|+|\mathfrak{G}|$. 
Let $-\mathcal{L}$ be those labels of $G_{Q}$ that are not in $\mathcal{L}$. The statements of this paragraph will all be up to an added linear function of $g$. The curves of intersection, denoted $a \theta(\Sigma)$, of $\widehat{F}$ with the long Möbius bands or long twisted $\theta$-bands coming from $\Sigma$, bound a collection of disjoint annuli of $\widehat{F}$. Each curve of $a \theta(\Sigma)$ corresponds to two elements of $L(\Sigma)$, so there are $|L(\Sigma)| / 2$ such annuli. Furthermore, half of these annuli must contain in their interiors vertices of $G_{F}$ which correspond to labels in $G_{Q}$ which are in either $-\mathcal{L}$ or $\mathfrak{G}$. This is Lemma 8.3. whose main ingredient is Lemma 5.9, This subcollection of annuli is called $\mathcal{A}$, and from the above we get $|\mathcal{A}| \geq|L(\Sigma)| / 4$. On the other hand, it follows from Lemma 7.8 and Corollary 7.9 that each simple gnarl must contain at least three vertices of $G_{F}$ corresponding to labels in $-\mathcal{L}$. Counting those elements of $-\mathcal{L}$ coming from from the interiors of the annuli in $\mathcal{A}$ and the gnarls in $\mathfrak{G}$ we estimate (equation sequence $\left(^{* *}\right)$ in section 8) that $|-\mathcal{L}| \geq(3 / 7)(|L(\Sigma)|+|\mathfrak{G}|) \geq(3 / 7)|\mathcal{L}| \geq(3 / 7)(3 / 4) t$.

Thus $t=|-\mathcal{L}|+|\mathcal{L}| \geq(3 / 4) t+(9 / 28) t-c(g)$, where $c(g)$ is a linear function of $g$, thereby bounding $t$ by $14 c(g)$.

The full proof of Theorem 8.1 is given at the beginning of section 8 , In the course of this proof, we keep track of and explicitly determine suitable linear bounds.

\section{FAT VERTEXED GRAPHS}

2.1. Heegaard splittings, thin position, and bridge position. In this paper, a Heegaard splitting will always be a 2-sided Heegaard splitting. Given such a Heegaard surface $S$ of a closed 3-manifold $Y$ there is a product $S \times \mathbb{R} \subset Y$ so that $S=S \times\{0\}$ and the complement of the product is the union of the cores of the two handlebodies. This defines a height function on the complement of the the cores of the handlebodies. Consider all the circles $C$ embedded in the product that are Morse with respect to the height function and represent the knot type of a particular knot $J$. The following terms are all understood to be taken with respect to the Heegaard splitting.

Following [10] (see also 32]), the width of an embedded circle $C$ is the sum of the number of intersections $\left|C \cap S \times\left\{y_{i}\right\}\right|$ where one regular value $y_{i}$ is chosen between each pair of consecutive critical values. The width of a knot $J$ is the minimum width of all such embeddings. An embedding realizing the width of $J$ is a thin position of $J$, and $J$ is said to be thin. If the critical point immediately below $y_{i}$ is a local minimum and the critical point immediately above $y_{i}$ is a local maximum, then the level $S \times\left\{y_{i}\right\}$ is a thick level. If the critical point immediately below $y_{i}$ is a local maximum and the critical point immediately above $y_{i}$ is a local minimum, then the level $S \times\left\{y_{i}\right\}$ is a thin level.

The minimal number of local maxima among Morse embeddings of $C$ is the bridge number of $J$, denoted $b r_{S}(J)$, or, if $S$ is understood, $b r(J)$. An embedding realizing the bridge number of $J$ may be ambient isotoped so that all local maxima lie above all local minima, without introducing any more extrema. The resulting embedding is a bridge position of $J$, and $J$ is said to be bridge.

With $J$ in bridge position, the arcs of $J$ intersecting a Heegaard handlebody are collectively $\partial$-parallel. There is an embedded collection of disks in the handlebody such that the boundary of each is formed of one $\operatorname{arc}$ on $S$ and one $\operatorname{arc}$ on $J$. A single such disk is called a bridge disk for that arc of $J$, and the arc is said to be bridge. 
A thin position for a knot may have smaller width than that of its bridge position, with respect to the same Heegaard splitting. That is, thin position may not be bridge position. However, this only happens when the meridian of the knot in the ambient manifold is a boundary slope of the knot exterior. Recall

Definition 2.1. If $E$ is a compact, orientable 3-manifold with torus boundary, a slope $r$ on $\partial E$ is a $(g, b)$-boundary slope for $E$ if there is a compact, connected, orientable essential surface $(F, \partial F) \subset(E, \partial E)$ with non-empty boundary and boundary slope $r$, such that $F$ has genus at most $g$ and at most $b$ boundary components. We say that $r$ is a $g$-boundary slope if it is a $(g, b)$-boundary slope for some $b$.

Lemma 2.2. Assume $J$ is a knot in a 3-manifold $M$. If $J$ has a thin position which is not a bridge position with respect to a genus $g$ Heegaard splitting of $M$, then the meridian of $J$ is a $(g, b)$-boundary slope for the exterior of $J$, where $b$ is the number of intersections of $J$ with any thin level surface in this thin presentation of $J$.

Proof. This is proved in 32] when $g=0$. The same proof works here. We sketch it for the convenience of the reader.

Let $S$ be the Heegaard surface of a genus $g$ splitting of $M$ with respect to which $J$ is in thin position but not bridge position. Then this thin presentation must have a thin level, $y$. Let $b$ be the number of intersections of $J$ with the thin level surface $S \times\{y\}$. There can be no bridge disks for $J$ to the thin level surface, otherwise such a disk would give rise to a thinner presentation of $J$. Maximally compress $(S \times\{y\})-\operatorname{Nbhd}(J)$ in the exterior of $J$. Either some component of the result is an incompressible, $\partial$-incompressible surface of genus at most $g$ with at most $b$ boundary components each of which is a meridian of $J$, or the result is a non-empty collection of boundary parallel annuli along with some closed surfaces. But each boundary parallel annulus gives rise to a bridge disk of $J$ onto $S \times\{y\}$, which is not possible. Thus the meridian is a $(g, b)$-boundary slope for the exterior of $J$.

Lemma 2.3. Assume $K^{\prime}$ is a non-trivial knot in $S^{3}$ with exterior $X=S^{3}-$ $\operatorname{Nbhd}\left(K^{\prime}\right)$. Let $M=K^{\prime}(p / q)$ and $K$ be the core of the attached solid torus in $M$. Suppose $M$ has a genus $g$ Heegaard surface and that $K$ cannot be isotoped to lie on this surface. Assume $K$ is in thin position with respect to this genus $g$ Heegaard splitting of $M$ and let $\widehat{F}$ be a corresponding thick level surface. Then there is a punctured 2-sphere $Q$ and a punctured genus $g$ surface $F$ properly embedded and transverse in $X$ satisfying the following:

(1) Each component of $\partial Q$ is a meridian of $\partial X$ and each component of $\partial F$ is a $p / q$ curve on $\partial X$

(2) Each arc of $F \cap Q$ is essential in each of $F$ and $Q$.

(3) There are no simple closed curves of $F \cap Q$ trivial in both $F$ and $Q$.

(4) Capping off $F$ with disks in $K^{\prime}(p / q)$ gives $\widehat{F}$. Capping off $Q$ with meridians of $K^{\prime}$ gives a 2 -sphere, $\widehat{Q}$, in $S^{3}$.

Proof. Let $K^{\prime}, X, M, \widehat{F}$ be as stated. Let $K$ be the core of the attached solid torus in $M=K^{\prime}(p / q)$. Isotop $K$ to be in thin position with respect to the given genus $g$ splitting of $M$ so that $\widehat{F}$ is a thick level surface. In $S^{3}$, put $K^{\prime}$ into thin position with respect to the genus 0 Heegaard splitting. By Theorem 6.2 of [26] (by assumption $K, K^{\prime}$ cannot be isotoped onto their Heegaard surfaces), there exists a thick level surface $\widehat{Q}$ of $S^{3}$ such that each arc of $F \cap Q$ is essential in each of $F=\widehat{F}-\operatorname{Nbhd}(K)$ 
and $Q=\widehat{Q}-\operatorname{Nbhd}\left(K^{\prime}\right)$. Note that the argument of Rieck's Theorem 6.2 allows us to choose the fat layer with which we want to work. Within the chosen fat layer, the intersection of any thick level surface with $X$ is properly isotopic in $X$ to the intersection of any other thick level surface with $X$. As the exterior of $K^{\prime}$ is irreducible, after an isotopy in $X$ we may assume there are no simple closed curves of $F \cap Q$ trivial in both $F$ and $Q$.

2.2. The graphs $G_{F}, G_{Q}$ and their combinatorics. In this section we describe the graphs $G_{F}, G_{Q}$ and the great web $\Lambda$, which will be the context of the rest of the paper. Let $F, Q$ be as in Lemma 2.3. On $\widehat{Q}$ and $\widehat{F}$ form the fat vertexed graphs of intersection, $G_{Q}$ and $G_{F}$, respectively, consisting of the fat vertices that are the disks $\operatorname{Nbhd}\left(K^{\prime}\right) \cap \widehat{Q}$ and $\operatorname{Nbhd}(K) \cap \widehat{F}$ and edges that are the arcs of $F \cap Q$.

Choosing an orientation on $K \subset M$, we may number the intersections of $K$ with $\widehat{F}$, and hence the vertices of $G_{F}$, from 1 to $t=|K \cap \widehat{F}|$ in order around $K$. Similarly, if $\left|K^{\prime} \cap \widehat{Q}\right|=u$, by choosing an orientation on $K^{\prime} \subset S^{3}$ we may number the intersections of $K^{\prime}$ with $\widehat{Q}$ and hence the vertices of $G_{Q}$ from 1 to $u$ in order around $K^{\prime}$.

Each component of $\partial F$ intersects each component of $\partial Q$ a total of $q$ times. Thus a vertex of $G_{Q}$ has valence $q t$ and a vertex of $G_{F}$ has valence $q u$. Since each component of $\partial F \cap \partial Q$ is an endpoint of an arc of $F \cap Q$, each endpoint of an edge in $G_{Q}$ may be labeled with the vertex of $G_{F}$ whose boundary contains the endpoint. Thus around the boundary of each vertex of $G_{Q}$ the labels $\{1, \ldots, t\}$ appear in order $q$ times. Similarly around the boundary of each vertex of $G_{F}$ the labels $\{1, \ldots, u\}$ appear in order $q$ times.

See the expository article 12 for a more thorough discourse on such fat vertexed graphs and standard techniques in their use.

Endow each vertex of $G_{Q}$ (and $G_{F}$ ) with a sign of + or - according to whether or not the corresponding component of $\partial Q(\partial F)$ with its induced orientation is parallel or anti-parallel on $\partial X$ to a chosen component of $\partial Q(\partial F)$. Two vertices on the same graph are parallel if they have the same sign, and anti-parallel if they have opposite signs.

The orientability of $F$ and $Q$ and the knot exterior gives the following useful property of these graphs.

Parity Rule: An edge connects parallel vertices on one of $G_{F}, G_{Q}$ iff it connects anti-parallel vertices on the other.

Definition 2.4. For a subgraph, $\Lambda$, of $G_{Q}$, a ghost edge for $\Lambda$ is an edge of $G_{Q}$ which does not belong to $\Lambda$ but is incident to a vertex of $\Lambda$. The incidence of a ghost edge for $\Lambda$ with a fat vertex of $\Lambda$ is called a ghost label of $\Lambda$. A connected subgraph, $\Lambda$, of $G_{Q}$ is called a $g-w e b$ if its vertices are parallel and if $\Lambda$ has at most $t+2 g-2$ ghost labels. If $U$ is a component of $\widehat{Q}-\Lambda$, then we say $D=\widehat{Q}-U$ is a disk bounded by $\Lambda$. A great $g-w e b$ is a $g$-web with the property that there is a disk, $D$, bounded by $\Lambda$ such that $\Lambda=G_{Q} \cap D$. Note that as long as $t>2 g-2$ and $q \geq 2$, then a great $g$-web must contain at least two vertices $\left(G_{Q}\right.$ has no 1 -sided faces).

Lemma 2.5. Let $G_{Q}, G_{F}$ be the graphs of intersection coming from $Q, F$ of Lemma 2.3 as described above. If $t>2 g-2$ and $q \geq 2$, then $G_{Q}$ has a great $g-w e b, \Lambda$. 
Proof. This is Theorem 6.1 of [12] where $\widehat{F}, t$ play the role of $\widehat{P}, p$, where the slope $\beta$ there is the meridional slope of $K^{\prime}$. In [12, a great $g$-web is not necessarily connected, but by restricting to a connected component we may take it to be.

The goal of Theorem 8.1 is to bound $K \cap \widehat{F}$ in terms of the genus of $\widehat{F}$. Thus, after Lemma 2.5 we will hereafter assume the existence in $G_{Q}$ of the great $g$-web, $\Lambda$.

Definition 2.6. For each label $x \in\{1, \ldots, t\}$, the subgraph of a great $g$-web $\Lambda$ consisting of all edges with an endpoint labeled $x$ and the vertices to which these edges are incident is denoted $\Lambda_{x}$. We think of $\Lambda_{x}$ as a graph in the disk bounded by $\Lambda$. A ghost edge of $\Lambda$ which is incident to a vertex of $\Lambda$ with label $x$ is called a ghost $x$-edge for $\Lambda$. By the Parity Rule, each ghost $x$-edge has at most one endpoint at a fat vertex of $\Lambda$ with label $x$. We refer to this endpoint as a ghost $x$-label. Let $\alpha_{x}$ denote the number of ghost $x$-labels for $\Lambda$.

Note that, taken over all labels, $\sum \alpha_{x} \leq t+2 g-2$.

Definition 2.7. A bigon (trigon) face of $\Lambda_{x}$ is referred to as an $x$-bigon (x-trigon, resp.) of $\Lambda$. Let $\mathcal{L}$ be the set of labels $x$ of $\Lambda$ for which $\Lambda$ has an $x$-bigon or $x$-trigon. By $-\mathcal{L}$ we denote the complement of $\mathcal{L}$ in the set of all labels of $G_{Q}$.

Lemma 2.8. If $q \geq 2, t>2 g-2$, and $\alpha_{x} \leq 3$, then $x \in \mathcal{L}$.

Proof. Assume that $q \geq 2, t>2 g-2$ and $\alpha_{x} \leq 3$. Assume for contradiction that $x \notin \mathcal{L}$. Take a connected component, $\Lambda_{x}^{\prime}$, of $\Lambda_{x}$ which is innermost in the disk $D$ bounded by $\Lambda$ and which has the least number of ghost $x$-labels among such components. Let $V, E, F$ be the number of vertices, edges, and faces of $\Lambda_{x}^{\prime}$ in the disk $D$. Let $n$ be the number of outside edges of $\Lambda_{x}^{\prime}$, counted with multiplicity. By assumption $\Lambda_{x}^{\prime}$ can contain no bigon or trigon face, so counting edges gives $2 E \geq 4 F+n$. Note that $E>0, n>0$ by our assumptions, and $\Lambda$ has at least two vertices. As $\alpha_{x} \leq 3$ and as each edge of $\Lambda$ has at most one label $x$ (Parity Rule), we have $(*) E \geq q V-3$. Along with the Euler characteristic equation $V-E+F=1$, these imply that $(E+3) / q-E+(2 E-n) / 4 \geq 1$. That is, $(2-q) E \geq(n+4) q / 2-6$. This implies that $q=2$ and $n \leq 2$. If $\Lambda_{x}^{\prime}$ has at most two ghost $x$-labels, then $E \geq q V-2$. Using this in place of $(*)$, the calculations above show that $(2-q) E \geq(n+4) q / 2-4$, a contradiction. Thus we have that $\Lambda_{x}^{\prime}$ has exactly 3 ghost $x$-labels. This implies that $\alpha_{x}=3$ and $\Lambda_{x}^{\prime}$ contains all the vertices of $\Lambda$. This along with the fact that $n \leq 2, q=2$ implies that $\Lambda_{x}^{\prime}$ consists of a single edge connecting two vertices and that one of these vertices has two ghost $x$-labels. Thus $\Lambda$ consists of two vertices connected by fewer than $t$ parallel edges ( $G_{Q}$ contains no $1-$ sided faces). As $q=2, \Lambda$ has more than $2 t$ ghost labels. Thus $2 t<t+2 g-2$, a contradiction.

Proposition 2.9. If $t>2 g-2$ and $q \geq 2$, then $|\mathcal{L}| \geq(3 / 4) t-(g-1) / 2$.

Proof. Assume $t>2 g-2$ and $x$ is a label which is not in $\mathcal{L}$. Then by Lemma 2.8 , $\alpha_{x} \geq 4$. Hence

$$
t+2 g-2 \geq \sum_{x} \alpha_{x}=\sum_{x \in \mathcal{L}} \alpha_{x}+\sum_{x \notin \mathcal{L}} \alpha_{x} \geq \sum_{x \notin \mathcal{L}} \alpha_{x} \geq 4|-\mathcal{L}|=4(t-|\mathcal{L}|) .
$$

Hence $|\mathcal{L}| \geq t-(t+2 g-2) / 4=(3 / 4) t-(g-1) / 2$. 


\subsection{Bigons and trigons of $\Lambda_{x}$.}

Definition 2.10. A Scharlemann cycle (of length $n$ ) is a disk face of $G_{Q}$ or $G_{F}$ with $n$ edges, all edges having the same pair of labels and all connecting parallel vertices of the graph. We use the same term for the set of edges defining the face. The Scharlemann cycles considered in this paper are typically on $G_{Q}$ and of length 2 or 3 .

Remark 2.11. Note that by the Parity Rule, any edge of the great $g$-web $\Lambda$ must have different labels in $G_{Q}$.

Definition 2.12. The subgraph of $\Lambda$ bounded by a bigon face in $\Lambda_{x}$ contains a Scharlemann cycle of length 2 . This subgraph is called an extended Scharlemann cycle of length 2, and the Scharlemann cycle it contains is referred to as its core Scharlemann cycle.

Definition 2.13. A trigon in $\Lambda_{x}$ is a cycle trigon if its edges can be oriented consistently around the trigon so that the $x$-label is always at the tail end of an edge (i.e., it forms an $x$-cycle). See Figure 13. Otherwise it is a non-cycle trigon. See Figure 14.

Definition 2.14. If $\sigma$ is a trigon face of $\Lambda_{x}$, then the subgraph of $\Lambda$ bounded by $\sigma$ consists of a trigon face of $\Lambda$ together with three arms, each consisting of a (possibly empty) string of bigon faces of $\Lambda$; see for example Figure 13

Definition 2.15. The subgraph of $\Lambda$ bounded by a trigon face, $\sigma$, in $\Lambda_{x}$, either contains a single Scharlemann cycle of length 3 or contains one or two Scharlemann cycles of length 2 (e.g. see the proof of Lemma 6.1). If it contains a Scharlemann cycle of length 3, this subgraph is called an extended Scharlemann cycle of length 3 , and the Scharlemann cycle it contains is referred to as its core Scharlemann cycle.

Remark 2.16. Trigons of $\Lambda_{x}$ which are not extended Scharlemann cycles will be classified later as Type I, II, or III (see Definition 6.2).

Definition 2.17. The arc on the boundary of a vertex between two consecutive edges of a face of a subgraph of $G_{Q}$ or $G_{F}$ is a corner of that face.

Definition 2.18. If a set of edges $\sigma$ is the boundary of a face $f$ of $\Lambda_{x}$, then let $L(\sigma)$ be the set of labels of $\Lambda$ that appear on the corners of $f$.

Definition 2.19. Let $\sigma$ be an extended Scharlemann cycle of $G_{Q}$. Let $\Gamma_{\sigma}$ be the subgraph of $G_{F}$ consisting of the outermost (in $G_{Q}$ ) edges of $\sigma$ and the vertices to which these edges are incident. We say $\sigma$ lies in a disk if $\Gamma_{\sigma}$ is contained in a disk in $\widehat{F}$. We say $\sigma$ lies in an essential annulus if $\Gamma_{\sigma}$ is contained in an annulus in $\widehat{F}$ but does not lie in a disk.

Lemma 2.20. No extended Scharlemann cycle of $G_{Q}$ lies in a disk.

Proof. Assume $\sigma$ is an extended Scharlemann cycle of length $p$ that lies in a disk. Note that $p>1$. Let $f$ be the face of $\sigma$. Let $D$ be a small disk in $\widehat{F}$ in which $\Gamma_{\sigma}$ lies (above). Consider the family $\mathcal{F}(\sigma)$ of extended Scharlemann cycles contained in $f$ with the same core Scharlemann cycle as $\sigma$ (excluding $\sigma$ but including the core Scharlemann cycle). If any of these lie in $D$, then we choose an innermost such, say $\sigma^{\prime}$, and replace $\sigma$ by $\sigma^{\prime}$. So we may assume that no element of $\mathcal{F}(\sigma)$ lies in $D$. By disk exchanges we may assume Int $f \cap D=\emptyset$. Let $H$ be the 1 -handle neighborhood 
of the arc of $K$ that forms the corners of $f$. Then $H$ meets $D$ only in the two vertices corresponding to the labels of the edges of $\sigma$, and $\operatorname{Nbhd}(D \cup f \cup H)$ is a punctured lens space of order $p$. This cannot happen since $M$ is neither reducible nor a lens space.

Lemma 2.21. No simple closed curve of $Q \cap F$ that is trivial in $Q$ is trivial in $\widehat{F}$.

Proof. Otherwise let $\widehat{D} \subset \widehat{F}$ be the disk bounded by such a simple closed curve. Let $G_{D}$ be $G_{F}$ restricted to $\widehat{D}$. By Lemma 2.3 (3), $G_{D}$ is non-empty. By Lemma 2.3 (3), there are no 1 -sided faces in $G_{D}$ and no 1 -sided faces in the subgraph of $G_{Q}$ corresponding to the edges of $G_{D}$. The argument of Proposition 2.5.6 of [7, along with the assumption that $q \geq 2$, implies that one of $G_{D}$ or $G_{Q}$ contains a Scharlemann cycle. Such a Scharlemann cycle along with the argument of Lemma 2.20 above would imply the contradiction that either $S^{3}$ or $M$ contains a lens space summand.

\section{Collections of curves with Property $P(k)$ and $F_{k}(g)$}

Definition 3.1. Let $\mathcal{C}$ be a collection of simple loops on a surface $S$, and let $k$ be a non-negative integer. We say that $\mathcal{C}$ has Property $P(k)$ if

(1) the elements of $\mathcal{C}$ are essential and pairwise non-isotopic on $S$;

(2) any pair of elements of $\mathcal{C}$ meet transversely;

(3) for all $c, c^{\prime} \in \mathcal{C},\left|c \cap c^{\prime}\right| \leq 1$; and

(4) any $c \in \mathcal{C}$ meets at most $k$ elements of $\mathcal{C}-\{c\}$.

For $k \geq 0$ and $g \geq 2$ define $F_{k}(g)=\max \{|\mathcal{C}|: \mathcal{C}$ is a collection of simple loops on a closed, connected, orientable surface of genus $g$ with Property $P(k)\}$.

Lemma 3.2. $F_{k}(g) \leq A_{k}(g-2)+B_{k}$, where $A_{k}=k+2\lfloor k / 4\rfloor+3$ and $B_{k}$ is:

$$
\begin{aligned}
3, & \text { if } k=0 ; \\
5, & \text { if } k=1 ; \\
7, & \text { if } k=2 \text { or } 3 ; \\
9, & \text { if } k=4 ; \\
10, & \text { if } k=5 ; \\
12, & \text { if } k \geq 6 .
\end{aligned}
$$

Moreover, we have equality if $g=2$ or $k=0,1$ or 2, i.e.,

$$
\begin{aligned}
& F_{k}(2)=B_{k} \text { for all } k, \text { and } \\
& F_{0}(g)=3 g-3, \\
& F_{1}(g)=4 g-3 \\
& F_{2}(g)=5 g-3 .
\end{aligned}
$$

Remark 3.3. In the present paper we will only make use of the formula for $F_{2}(g)$ (and $F_{0}(g)$ ). However, we include the general case as possibly being of independent interest.

Proof. We will prove the lemma by induction on $g$. Since the case $k=0$ is easy and well known, we assume $k \geq 1$. Let $\mathcal{C}$ be a collection of simple loops with Property $P(k)$ on a closed, connected, orientable surface $S$ of genus $g \geq 2$. 
First we consider the special case where there is a loop $c_{0} \in \mathcal{C}$ that bounds a once-punctured torus $T_{0} \subset S$ containing a curve in $\mathcal{C}$ that intersects $k$ other elements of $\mathcal{C}$. Let $S_{0}$ be the other component of $S$ cut along $c_{0}$, and let $S^{\prime}$ be the closed surface of genus $g-1$ obtained by capping off the boundary of $S_{0}$ with a disk $D_{0}$. Note that $c_{0}$ is disjoint from all the other members of $\mathcal{C}$. It follows that either $k=1$ and there are exactly two elements of $\mathcal{C}$ in $\operatorname{Int} T_{0}$, or $k=2$ and there are exactly three elements of $\mathcal{C}$ in Int $T_{0}$. Discarding from $\mathcal{C}$ these elements, together with $c_{0}$, we get a collection $\mathcal{C}_{0}$ of loops on $S_{0}$. Regarded as a collection of loops on $S^{\prime}, \mathcal{C}_{0}$ satisfies all the conditions for Property $P(k)$ except possibly $(1)$ : some pairs of loops in $\mathcal{C}_{0}$ may become isotopic on $S^{\prime}$. (Note that no loop in $\mathcal{C}_{0}$ is inessential in $S^{\prime}$, as such a loop would be parallel to $c_{0}$ in $S$.)

Let $a, a^{\prime} \in \mathcal{C}_{0}$ be curves that are isotopic on $S^{\prime}$. Then $a$ and $a^{\prime}$ are disjoint, and cobound an annulus $A$ in $S^{\prime}$. Since $a$ and $a^{\prime}$ are not isotopic on $S$, we have $D_{0} \subset \operatorname{Int} A$. In particular, it follows that no triple of curves in $\mathcal{C}_{0}$ is isotopic in $S^{\prime}$. Now suppose $b, b^{\prime} \in \mathcal{C}_{0}$ is another pair of curves that are isotopic in $S^{\prime}$. Then $b$ and $b^{\prime}$ cobound an annulus $B$ in $S^{\prime}$ with $D_{0} \subset$ Int $B$. Hence $A \cap B \neq \emptyset$. If $\partial A \cap \partial B=\emptyset$, then either $a$ or $a^{\prime}$ lies in $B$, or $b$ or $b^{\prime}$ lies in $A$. But then $a$ or $a^{\prime}$ would be isotopic to $b$ or $b^{\prime}$ in $S$, a contradiction. Hence, without loss of generality, $a \cap b \neq \emptyset$. Therefore $b \cap A$ is a single transverse arc in $A$. Since $b$ and $b^{\prime}$ are isotopic in $S^{\prime}$, we must also have $a \cap b^{\prime} \neq \emptyset$, and so $b^{\prime} \cap A$ is also a single transverse arc. Thus the pair $b, b^{\prime}$ contributes two points of intersection to each of $a$ and $a^{\prime}$.

It follows that if $k=1$, then at most one pair of curves in $\mathcal{C}_{0}$ are isotopic in $S^{\prime}$. We thus get a collection $\mathcal{C}^{\prime}$ of loops on $S^{\prime}$ with Property $P(1)$ such that $\left|\mathcal{C}^{\prime}\right| \geq\left|\mathcal{C}_{0}\right|-1$. Therefore $|\mathcal{C}| \leq\left|\mathcal{C}_{0}\right|+3 \leq\left|\mathcal{C}^{\prime}\right|+4$. By induction $\left|\mathcal{C}^{\prime}\right| \leq 4(g-1)-3$, and hence $|\mathcal{C}| \leq 4 g-3$ as claimed.

If $k=2$ and at most one pair of curves in $\mathcal{C}_{0}$ are isotopic in $S^{\prime}$, then the result follows easily by induction. So suppose that two pairs of curves $a, a^{\prime}$ and $b, b^{\prime}$ in $\mathcal{C}_{0}$ become isotopic in $S^{\prime}$. Let $A, B$ be the annuli cobounded by $a, a^{\prime}$ and $b, b^{\prime}$ respectively, extended slightly so that $a \subset \operatorname{Int} A$ and $b \subset \operatorname{Int} B$. Then $A \cap B$ is a disk containing $D_{0}$, and hence $A \cup B$ is a once-punctured torus. There is a simple loop $c$ in $A \cup B$ intersecting each of $a$ and $b$ transversely in a single point. Then $\mathcal{C}^{\prime}=\left(\mathcal{C}-\left\{a^{\prime}, b^{\prime}\right\}\right) \cup\{c\}$ is a collection of loops on $S^{\prime}$ with Property $P(2)$. Hence, using the inductive hypothesis,

$$
\begin{aligned}
|\mathcal{C}| & \leq\left|\mathcal{C}_{0}\right|+4 \\
& \leq\left(\left|\mathcal{C}^{\prime}\right|+2-1\right)+4=\left|\mathcal{C}^{\prime}\right|+5 \\
& \leq(5(g-1)-3)+5=5 g-3 .
\end{aligned}
$$

We now consider the general case, and assume that $\mathcal{C}$ contains no loop $c_{0}$ as above. Since $A_{k}(g-2)+B_{k}$ is non-decreasing in $k$, we may assume that $\mathcal{C}$ does not have Property $P(k-1)$, i.e., there is a loop $c_{0} \in \mathcal{C}$ that intersects $k$ other elements $c_{1}, c_{2}, \ldots, c_{k} \in \mathcal{C}$. In particular, $c_{0}$ is non-separating on $S$, and so cutting $S$ along $c_{0}$ gives a connected surface $S_{0}$. Let $S^{\prime}=S_{0} \cup D_{1} \cup D_{2}$ be the closed surface of genus $(g-1)$ obtained by capping off the two boundary components of $S_{0}$ with disks $D_{1}$ and $D_{2}$. Let $S_{1}=S_{0} \cup D_{1}$.

Let $\mathcal{C}_{1}=\mathcal{C}-\left\{c_{0}, c_{1}, \ldots, c_{k}\right\}$. As a collection of loops on $S_{1}, \mathcal{C}_{1}$ satisfies Property $P(k)$ except that some pairs of loops in $\mathcal{C}_{1}$ may become isotopic in $S_{1}$. (Again, no loop in $\mathcal{C}_{1}$ is trivial in $S_{1}$, as such a loop would be parallel to $c_{0}$ in $S$.) Let $a, a^{\prime}$ be such a pair. The previous discussion applies verbatim, with $\mathcal{C}_{0}$ replaced 
by $\mathcal{C}_{1}$ and $S^{\prime}$ replaced by $S_{1}$, so any other such pair $b, b^{\prime}$ contributes two points of intersection to each of $a$ and $a^{\prime}$.

At least $\left\lfloor\frac{k+1}{2}\right\rfloor$ of the curves $c_{1}, c_{2}, \ldots, c_{k}$ must intersect one of $a, a^{\prime}$. Hence the number of pairs of elements of $\mathcal{C}_{1}$ that are isotopic in $S_{1}$ is at most $1+\left\lfloor\frac{k-\left\lfloor\frac{k+1}{2}\right\rfloor}{2}\right\rfloor=$ $1+\left\lfloor\frac{k}{4}\right\rfloor$. Discarding one element of $\mathcal{C}_{1}$ from each such pair, we get a collection of loops $\mathcal{C}_{2}$ with Property $P(k)$ in $S_{1}$. Now apply the same argument to $\mathcal{C}_{2}$, regarded as a collection of loops in $S^{\prime}=S_{1} \cup D_{2}$. We may assume that no element of $\mathcal{C}_{2}$ is inessential in $S^{\prime}$, as such a curve $c$ would bound a disk $D$ in $S^{\prime}$ with $D_{1} \cup D_{2} \subset \operatorname{Int} D$, and would therefore bound a once-punctured torus in $S$ containing $c_{0}$, putting us in the special case treated earlier. We then get a collection $\mathcal{C}^{\prime}$ of loops in $S^{\prime}$ satisfying Property $P(k)$, with $\left|\mathcal{C}^{\prime}\right| \geq\left|\mathcal{C}_{1}\right|-2(1+\lfloor k / 4\rfloor)$. Hence

$$
\begin{aligned}
|\mathcal{C}| & =\left|\mathcal{C}_{1}\right|+(k+1) \\
& \leq\left|\mathcal{C}^{\prime}\right|+(k+1)+2(1+\lfloor k / 4\rfloor) \\
& =\left|\mathcal{C}^{\prime}\right|+A_{k} .
\end{aligned}
$$

Now assume $g \geq 3$ and that the theorem holds for $(g-1)$. Then

$$
|\mathcal{C}| \leq\left(A_{k}(g-3)+B_{k}\right)+A_{k}=A_{k}(g-2)+B_{k},
$$

and the result follows by induction on $g$.

To start the induction we will show that $F_{k}(2)=B_{k}$. Let $S$ be a closed, orientable surface of genus 2 and let $\mathcal{C}$ be a collection of simple loops on $S$ with Property $P(k)$. Let $\tau: S \rightarrow S$ be the hyperelliptic involution. The quotient $(S, \operatorname{Fix}(\tau)) / \tau \cong\left(S^{2}, V\right)$, where $V$ consists of six points. Let $\pi: S \rightarrow S^{2}$ be the quotient map. The elements of $\mathcal{C}$ can be isotoped so that $\tau(c)=c$ for all $c \in \mathcal{C}$. If $c$ meets $\operatorname{Fix}(\tau)$, then $\pi(c)$ is an arc in $S^{2}$ whose endpoints are distinct points in $V$; if $c \cap \operatorname{Fix}(\tau)=\emptyset$, then $\pi(c)$ is a simple loop in $S^{2}-V$. By part (3) of Definition 3.1, if $c, c^{\prime}$ are distinct elements of $\mathcal{C}$, then $\pi(c) \cap \pi\left(c^{\prime}\right)$ is either empty or a single point in $V$. The $\operatorname{arcs} \pi(c)$ form the edges of a graph $\Gamma$ in $S^{2}$ with vertex set $V$, and $\pi(\cup \mathcal{C})$ is the disjoint union $\Gamma \amalg \Lambda$ where the components of $\Lambda$ are simple loops. Hence $|\mathcal{C}|=E(\Gamma)+|\Lambda|$, where $E(\Gamma)$ is the number of edges of $\Gamma$. Note that no two edges of $\Gamma$ share the same pair of endpoints, no component of $\Lambda$ bounds a disk in $S^{2}-V$, and no two components of $\Lambda$ are parallel in $S^{2}-V$. Finally, by part (4) of Definition 3.1, the sum of the valencies of the vertices at the endpoints of each edge of $\Gamma$ is at most $k+2$.

It is straightforward to check that with these constraints, for $0 \leq k \leq 5$ the maximum value of $E(\Gamma)+|\Lambda|$ is the $B_{k}$ given in the statement of the lemma. These values are realized by the configurations shown in Figure1. Note that by passing to the 2 -fold branched covering of $\left(S^{2}, V\right)$ we obtain a collection $\mathcal{C}$ of simple loops on $S$ with Property $P(k)$. For $k=6$ we have the 1-skeleton of the octahedron shown in Figure 1, with 12 edges. An easy Euler characteristic argument shows that a graph $\Gamma$ in $S^{2}$ with six vertices, no loop edges and no parallel edges, has at most 12 edges. It follows that $F_{k}(2)=12$ for $k \geq 6$.

Finally we show that the inequality in the lemma is an equality when $k=0,1$ or 2 . For $k=0$, this is easy and well known. For $k=1$ or 2 , let $S$ be the closed surface of genus $g$ obtained by attaching $g$ once-punctured tori $T_{i}, 1 \leq i \leq g$, to a $g$-punctured sphere $P$ along their boundaries. In each $T_{i}$ take a collection $\mathcal{C}_{i}$ of 2 (resp. 3 ) simple loops with pairwise intersection numbers equal to 1 , and 


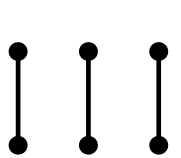

$k=0$

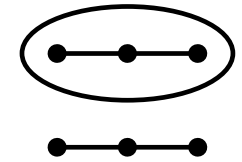

$k=1$

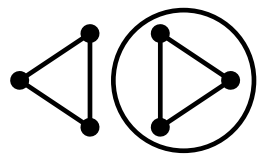

$k=2$
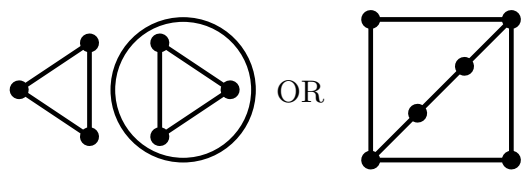

$k=3$

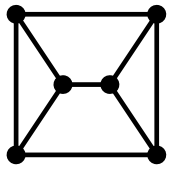

$k=4$

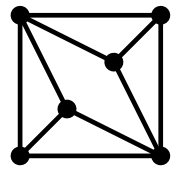

$k=5$

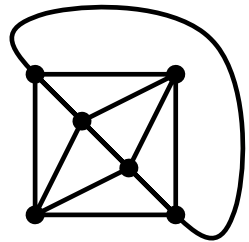

$k=6$

Figure 1

in $P$ take a collection $\mathcal{C}_{0}$ of simple loops that cut $P$ into pairs of pants. Define $\mathcal{C}=\left(\bigcup_{i=1}^{g} \mathcal{C}_{i}\right) \cup \mathcal{C}_{0} \cup\{$ components of $\partial P\}$, a collection of loops on $S$. Then $\mathcal{C}$ satisfies Property $P(1)$ (resp. $P(2))$ and $|\mathcal{C}|=2 g+(g-3)+g=4 g-3$ (resp. $3 g+(g-3)+g=5 g-3)$.

Remark 3.4. Consider the number $F(g)=\max _{k}\left\{F_{k}(g)\right\}$. 22 shows $g^{2}+g \leq$ $F(g) \leq(g-1) 2^{2 g}$ for $g \geq 2$. Moreover, by essentially the same method as the base case of our induction, $[22$ proves $F(2)=12$.

\section{Extended Scharlemann CyCles of LEngth 2 AND 3}

Definition 4.1. Let $\{a, b\}$ be labels of $G_{Q}$. Then $\overline{a b}$ is one of the two intervals of labels running between $a$ and $b$ on an abstract vertex of $G_{Q}$ that contains no labels $a, b$ on its interior. Typically, one such interval is specified by the context.

If $\sigma$ is an extended Scharlemann cycle whose edges have labels $\{a, b\}$, then we may think of $L(\sigma)$ as an interval of labels $\overline{a b}$.

Lemma 4.2. Let $\sigma, \tau$ be extended Scharlemann cycles of length 2 or 3 . Then either

(1) $L(\sigma) \cup L(\tau)$ contains all labels;

(2) $L(\sigma) \subset L(\tau)$ or $L(\tau) \subset L(\sigma)$;

(3) $L(\sigma) \cap L(\tau)$ is a single interval of labels $\overline{x y}$ where $x$ is an extremal label of $\sigma$ and $y$ is an extremal label of $\tau$; or

(4) $L(\sigma) \cap L(\tau)=\emptyset$.

Proof. $L(\sigma), L(\tau)$ are each a single interval of labels. By convexity, either $L(\sigma) \cup$ $L(\tau)$ contains all labels or $L(\sigma) \cap L(\tau)$ is convex. If non-empty, it is a single interval of intersection, $\overline{x y}$. Clearly, $x$ and $y$ must be extremal in either $\sigma$ or $\tau$. If both are extremal in, say, $\sigma$, then $L(\sigma)$ is contained in $L(\tau)$. 
Definition 4.3. Let $\sigma$ be an extended Scharlemann cycle of length 2 with $L(\sigma)=$ $\overline{x y}$. Let $f$ be the union of the bigons bounded by $\sigma$ in $G_{Q}$. Construct the long Möbius band $A(\sigma)$ in $M$ by extending the corners of $f$ radially to $K$ in a neighborhood of $K$ in $M$. Let $a(\sigma)$ be the set of simple closed curves $A(\sigma) \cap \widehat{F}$ that are formed by pairs of edges of $\sigma$. The core labels of $\sigma$ are the two labels of the Scharlemann cycle face of $\sigma$. The core curve of $\sigma$ is the element of $a(\sigma)$ on its core labels.

Definition 4.4. An embedded $\theta$-curve in a surface $\widehat{F}$ is a graph $\theta$ consisting of two vertices and three edges, between these two vertices, embedded in $\widehat{F}$ so that $\operatorname{Nbhd}(\theta)$ is a thrice-punctured sphere. A $\theta$-band is homeomorphic to the product of an embedded $\theta$-curve with an interval.

Lemma 4.5. Let $\sigma$ be a Scharlemann cycle of length 3 in $G_{Q}$. Then, regarding as points the two vertices of $G_{F}$ to which the edges of $\sigma$ are incident, the edges of $\sigma$ form an embedded $\theta$-curve in $\widehat{F}$.

Proof. Let $f$ be the trigon of $G_{Q}$ bounded by $\sigma$. Let $\{x, x+1\}$ be the label pair on the edges of $\sigma$. With $\alpha, \beta$, and $\gamma$ label the corners of $f$ and hence label the endpoints of the edges of $\sigma$ too. These labels then appear around vertices $x$ and $x+1$ of $G_{F}$ in opposite directions. Since each edge of $\sigma$ connects two distinct corners of $f$, the endpoints of each edge have distinct labels. Shrinking vertices $x$ and $x+1$ to points, this forces $\sigma$ to form an embedded $\theta$-curve in $\widehat{F}$.

Definition 4.6. Let $\sigma$ be an extended Scharlemann cycle of length 3 with $L(\sigma)=$ $\overline{x y}$. Let $f$ be the union of the bigons and the trigon bounded by $\sigma$ in $G_{Q}$. Construct the long twisted $\theta$-band $\Theta(\sigma)$ in $M$ by extending the corners of $f$ radially to the core of $H_{x y}$. Let $\theta(\sigma)$ be the set of embedded $\theta$-curves $\Theta(\sigma) \cap \widehat{F}$. The core labels of $\sigma$ are the two labels of the Scharlemann cycle face of $\sigma$. The core $\theta$-curve of $\sigma$ is the element of $\theta(\sigma)$ on its core labels.

The following shorthand will be convenient.

Definition 4.7. If $\sigma$ is an extended Scharlemann cycle of length 3, then take $A(\sigma)=\emptyset$ and $a(\sigma)=\emptyset$. If $\sigma$ is an extended Scharlemann cycle of length 2 , then take $\Theta(\sigma)=\emptyset$ and $\theta(\sigma)=\emptyset$. If $\sigma$ is an extended Scharlemann cycle of length 2 or 3 , let $a \theta(\sigma)=a(\sigma) \cup \theta(\sigma)$ and $A \Theta(\sigma)=A(\sigma) \cup \Theta(\sigma)$.

By an abuse of notation we often regard $A(\sigma), \Theta(\sigma)$, and $A \Theta(\sigma)$ as collections of their constituent Möbius bands and annuli or twisted $\theta$-bands and $\theta$-bands. So for example we will often speak of an annulus $A_{i} \in A(\sigma)$ bounded by $a_{i} \cup a_{i-1}$.

Definition 4.8. Let $\mathcal{E}=\{L(\sigma) \mid \sigma$ is an extended Scharlemann cycle of $\Lambda$ of length 2 or 3$\}$ be the set of all labels that lie on the corners of extended Scharlemann cycles of length 2 or 3 in $\Lambda$.

Definition 4.9. Let $\Sigma$ be a collection of extended Scharlemann cycles of length 2 or 3 in $\Lambda$ such that $L(\Sigma)=\cup\{L(\sigma) \mid \sigma \in \Sigma\}$ contains $\mathcal{E}$. Let $a \theta(\Sigma)=\bigcup_{\sigma \in \Sigma} a \theta(\sigma)$. Assume $\Sigma$ is chosen so that the complexity $(|\Sigma|,|a \theta(\Sigma)|\}$ is minimal in the lexicographic ordering among all such collections. 
Lemma 4.10. If $\sigma, \tau \in \Sigma$, then either

(1) $L(\sigma) \cup L(\tau)$ contains all labels;

(2) $L(\sigma) \cap L(\tau)$ is a single interval of labels $\overline{x y}$ where $x$ is an extremal label of $\sigma$ and $y$ is an extremal label of $\tau$; or

(3) $L(\sigma) \cap L(\tau)=\emptyset$.

Proof. If none of these were to occur, then by Lemma 4.2 the only other possibility is that either $L(\sigma) \subset L(\tau)$ or $L(\tau) \subset L(\sigma)$. Therefore $\sigma$ or $\tau$ respectively may be dropped from $\Sigma$ to produce $\Sigma^{\prime}$ so that $L\left(\Sigma^{\prime}\right)$ contains $\mathcal{E}$ and yet has lesser complexity than $\Sigma$. This contradicts the minimality assumption on $\Sigma$.

Definition 4.11. The extended Scharlemann cycle $\sigma^{\prime}$ is obtained by paring down the extended Scharlemann cycle $\sigma$ if $\sigma^{\prime}$ is contained in the disk face that $\sigma$ bounds.

Lemma 4.12. Two elements of a $(\Sigma)$ intersect at most once. Furthermore, each vertex of $G_{F}$ belongs to at most two different elements of $a \theta(\Sigma)$.

Proof. If there exists $\sigma, \tau \in \Sigma$ with $c_{\sigma} \in a \theta(\sigma)$ and $c_{\tau} \in a \theta(\tau)$ intersecting twice, then $c_{\sigma}$ and $c_{\tau}$ have the same label pair. Therefore we may pare down either $\sigma$ or $\tau$ (or completely eliminate one) to produce $\Sigma^{\prime}$ so that $L\left(\Sigma^{\prime}\right)$ contains $\mathcal{E}$ and yet has lesser complexity than $\Sigma$. This contradicts the minimality assumption on $\Sigma$.

If label $x$ of $G_{Q}$ were in $L\left(\sigma_{1}\right), L\left(\sigma_{2}\right)$ and $L\left(\sigma_{3}\right)$ with $\sigma_{1}, \sigma_{2}, \sigma_{3} \in \Sigma$, then one of these label sets must be contained in the union of the other two. This contradicts the minimality of $\Sigma$, thereby proving the final statement of the lemma.

Lemma 4.13. Let $\sigma$ be an extended Scharlemann cycle of length 3 that lies in an essential annulus $A$ of $\widehat{F}$. Let $f$ be the face bounded by $\sigma$ and assume $\operatorname{Int} f \cap A=\emptyset$. Then $N=\operatorname{Nbhd}(A \cup \Theta(\sigma))$ is a solid torus and the core of $A$ runs 3 times in the longitudinal direction of $N$.

Proof. Let $H$ be the 1-handle neighborhood of the arc of $K$ that forms the corners of $f$. Then $N=\operatorname{Nbhd}(A \cup \Theta(\sigma))$ may be obtained by attaching the 2-handle $\operatorname{Nbhd}(f)$ to the genus 2 handlebody $\operatorname{Nbhd}(A) \cup H$. Because a meridional disk of $\operatorname{Nbhd}(A)$ intersects $\partial f$ once, $N$ is a solid torus. One may then observe that the core of $A$ runs 3 times in the longitudinal direction of $N$. (Cf. Lemma 2.1 [11; Lemma 3.7 [16.)

Lemma 4.14. Let $\sigma$ be an extended Scharlemann cycle of length 2 or 3 in $\Lambda$. Assume an element of $a \theta(\sigma)$ lies in an annulus $A$ of $\widehat{F}$. Let $\eta$ be the core of $A$. Then $\eta$ cannot bound a disk in $M$ whose framing on $\operatorname{Nbhd}(\eta)$ is the same as that of $\widehat{F}$. In particular, $\eta$ is neither trivial on $\widehat{F}$ nor a meridian curve on either side of $\widehat{F}$.

Proof. Assume some element, $c$, of $a \theta(\sigma)$ lies in an annulus $A$ in $\widehat{F}$ whose core is $\eta$. Assume for contradiction that there is a disk $D$ in $Y=M-\operatorname{Nbhd}(\eta)$ whose boundary has the same slope on $\partial \operatorname{Nbhd}(\eta)$ as $\widehat{F}$. Pare down $\sigma$ so that $c$ is composed of the outermost edges of $\sigma$. The corners of $\sigma$ belong to the same subarc, $k$, of $K$. Let $f$ be the bigon or trigon face bounded by $\sigma$. Then $A \Theta(\sigma)$ (whose boundary is $c$ ) is $f$ radially contracted to $k$. We view both $A \Theta(\sigma)$ and $D$ as properly embedded in $Y$. By the framing assumption on $D$, we may take the boundaries of these two surfaces to be disjoint. Isotop $k$ rel its endpoints on $\partial Y$ so that $k$ intersects $D$ minimally. Then any arc components of intersection between $f$ and 
$D$ must be parallel on $f$ to the outermost edges of $\sigma$. We may surger away any simple closed curves of intersection. If $k$ is disjoint from $D$, then a neighborhood of $D \cup \operatorname{Nbhd}(\eta) \cup A \Theta(\sigma)$ will be a lens space summand of $M$ (using Lemma4.13 when the length of $\sigma$ is 3 ), a contradiction. If $k$ intersects $D$, then the intersection between $D$ and $f$ is a collection of arcs parallel to $\partial f$ with the same number of endpoints on each corner of $f$. An innermost face of this graph in $f$ will give a length 2 or 3 Scharlemann cycle with respect to $D$ which we can use, as in Lemma 2.20, to construct a lens space summand in $M$.

\subsection{Counting with extended Scharlemann cycles.}

Lemma 4.15. Let $\mathcal{T}$ be a set of embedded $\theta$-curves in $\widehat{F}$ such that

(1) no $\theta \in \mathcal{T}$ lies in a disk in $\widehat{F}$;

(2) if $\theta, \theta^{\prime} \in \mathcal{T}$, then $\theta \cap \theta^{\prime}$ is either empty or a single vertex;

(3) any vertex belongs to at most two elements of $\mathcal{T}$.

If $|\mathcal{T}|>3 F_{2}(g)$, then some $\theta \in \mathcal{T}$ lies in an essential annulus in $\widehat{F}$.

Proof. By (1), for each $\theta \in \mathcal{T}$ we may choose an essential circle $c_{\theta}$ in $\theta$ by deleting the interior of one of the edges of $\theta$. Let $\mathcal{C}=\left\{c_{\theta} \mid \theta \in \mathcal{T}\right\}$. If $\mathcal{C}^{\prime} \subset \mathcal{C}$ is a subcollection such that no two elements of $\mathcal{C}^{\prime}$ are isotopic in $\widehat{F}$, then (after isotoping the elements of $\mathcal{C}^{\prime}$ into general position) $\mathcal{C}^{\prime}$ has Property $P(2)$ of Definition 3.1. Hence, by Lemma 3.2 if $|\mathcal{C}|>3 F_{2}(g)$, then four elements of the $\mathcal{C}$ must be isotopic in $\widehat{F}$. Any two of these curves are either disjoint or intersect in a single vertex non-transversely. In either case we think of two such curves as cobounding an (essential) annulus in $\widehat{F}$. One sees that one of these four curves must contain a vertex that lies strictly in the interior of the annulus cobounded by two others. The corresponding $\theta$-curve then lies within this annulus.

\section{Thinning With eXtended Scharlemann CyCles}

Definition 5.1. Let $\sigma$ be an extended Scharlemann cycle of length 2 with $|a(\sigma)|=$ $n$. Then we may order the elements of $a(\sigma)$ as $a_{1}, \ldots, a_{n}$ such that $a_{1}$ is the core curve and $a_{i} \cup a_{i-1}$ bounds an annulus $A_{i} \subset A(\sigma)$ that is disjoint from the other elements of $a(\sigma)$ for $i=2, \ldots, n$. The core Scharlemann cycle in $\sigma$ forms the core curve $a_{1}$ which bounds the Möbius band $A_{1}$.

Definition 5.2. Let $\sigma$ be an extended Scharlemann cycle of length 3 with $|\theta(\sigma)|=$ $n$. Then we may order the elements of $\theta(\sigma)$ as $\theta_{1}, \ldots, \theta_{n}$ such that $\theta_{1}$ is the core $\theta$-curve and $\theta_{i} \cup \theta_{i-1}$ bounds a product $\theta$-curve $\Theta_{i} \subset \Theta(\sigma)$ that is disjoint from the other elements of $\theta(\sigma)$ for $i=2, \ldots, n$. The core Scharlemann cycle in $\sigma$ forms the core $\theta$-curve which bounds the twisted $\theta$-band $\Theta_{1}$.

Lemma 5.3. Let $\sigma$ be an extended Scharlemann cycle of length 3 . Assume $\theta_{i}, \theta_{j} \in$ $\theta(\sigma), i<j$, lie in an essential annulus $B$ in $\widehat{F}$. Exactly two of the edges, $e_{1}^{i}, e_{2}^{i}$ of $\theta_{i}$, cobound a disk in $\widehat{F}$. Let $e_{1}^{j}, e_{2}^{j}$ be the edges of $\theta_{j}$ that lie on the corresponding arms (Definition 2.14) of $\sigma$. Then $e_{1}^{j}, e_{2}^{j}$ cobound a disk on $\widehat{F}$.

Proof. Since $\theta_{i}$ lies in the annulus $B$, two of its edges, $e_{1}^{i}, e_{2}^{i}$, cobound a disk, $D$, in $B$, and (by Lemma 4.14) the third creates a core curve of $B$ when joined with either $e_{1}^{i}$ or $e_{2}^{i}$. For $k$ between (and including) $i$ and $j$, let $\theta_{k} \in \theta(\sigma)$ be as in Definition 5.2. Let $e_{1}^{k}, e_{2}^{k}$ be the edges of $\theta_{k}$ such that $e_{1}^{k}$ lies on the same arm of 
$\sigma$ as $e_{1}^{i}$, and $e_{2}^{k}$ on the same arm as $e_{2}^{i}$. Let $\gamma_{k}$ be the curve $e_{1}^{k} \cup e_{2}^{k}$. Let $F_{1}, F_{2}$ be the union of the faces on each arm between $e_{1}^{i}, e_{1}^{j}$ and $e_{2}^{i}, e_{2}^{j}$. Then $F_{1} \cup F_{2}$ forms an annulus $A$ in $M$ whose boundary components are $\gamma_{i}$ and $\gamma_{j}$.

By passing to a concentric subannulus of $B$, we may assume that $\partial B=\gamma_{i}^{\prime} \cup \gamma_{j}^{\prime}$, where $\gamma_{i}^{\prime}, \gamma_{j}^{\prime}$ are curves in $\theta_{i}, \theta_{j}$. Any component of $A \cap B$ is one of the curves $\gamma_{k}$ for some $k$ between $i$ and $j$. The corresponding $\theta$-curve $\theta_{k}$ is then contained in $B$, and does not lie in a disk by Lemma 4.14. Also, $\gamma_{k}$ is either a core curve of $B$ or bounds a disk in $B$.

Assume for contradiction that $\gamma_{j}$ does not bound a disk in $B$. Since $\gamma_{i}$ does bound a disk in $B$, there exist $k, k^{\prime}$ between $i$ and $j$ such that $\gamma_{k}$ and $\gamma_{k^{\prime}}$ are adjacent on $B$ among the components of $A \cap B, \gamma_{k}$ bounds a disk in $B$ and $\gamma_{k^{\prime}}$ is a core curve of $B$. By renumbering we may therefore assume that $k=i$ and $k^{\prime}=j$; thus the annulus $A$ meets $B$ only in $\partial A$. Let $E$ be the disk $A \cup D$. By pushing $D$ slightly off $B$ in the appropriate direction we may assume that $E \cap B=\partial E=\gamma_{j}$.

Let $l=\min \{i, j\}$ and let $\sigma^{\prime}$ be the extended Scharlemann cycle within $\sigma$ that terminates at the edges of $\theta_{l}$. Then the interior of the face bounded by $\sigma^{\prime}$ is disjoint from $B$ and $E$. Let $N$ be a regular neighborhood in $M$ of $\Theta\left(\sigma^{\prime}\right) \cup B \cup E$. By Lemma 4.13, $N$ is a punctured lens space of order 3, a contradiction.

The above lemma allows certain arguments for extended Scharlemann cycles of length 2 to apply to extended Scharlemann cycles of length 3 . To facilitate this we make the following definitions.

Definition 5.4. Assume $\sigma$ is an extended Scharlemann cycle of length 3 with $\theta_{i}, \theta_{j} \in \theta(\sigma), i<j$, lying in an essential annulus $B$ in $\widehat{F}$. Let $e_{1}^{i}, e_{2}^{i}$ be the edges of $\theta_{i}$ that cobound a disk, $D_{i}$, in $B$ and $e_{3}^{i}$ be the third edge of $\theta_{i}$. Let $e_{1}^{j}, e_{2}^{j}, e_{3}^{j}$ be the edges of $\theta_{j}$ on the corresponding arms of $\sigma$. By Lemma $5.3, e_{1}^{j}, e_{2}^{j}$ cobound a disk $D_{j}$ in $B . D_{i}\left(D_{j}\right)$ is called the disk of parallelism for $\theta_{i}\left(\theta_{j}\right.$, resp.). For $k=1,2,3$, let $F_{k}$ be the faces along an arm of $\sigma$ between $e_{k}^{i}, e_{k}^{j}$. By Lemma 5.3. $A_{1}=F_{1} \cup F_{3}$ and $A_{2}=F_{2} \cup F_{3}$ form isotopic annuli in $M$ whose boundaries lie in $\theta_{i}, \theta_{j}$ and are isotopic to the core of $B$. $A_{1}, A_{2}$ are called the constituent annuli of $\Theta_{i} \cup \cdots \cup \Theta_{j}$ of $A \Theta(\sigma)$ between $\theta_{i}, \theta_{j}$.

Lemma 5.5. Let $\sigma$ be an extended Scharlemann cycle of length $m=2$ or $m=3$. Assume $a_{i}, a_{j} \in a \theta(\sigma), i<j$, lie on an annulus, $B$, in $\widehat{F}$ such that all of the intersections of $K$ with $B$ belong to $a_{i}, a_{j}$ (i.e., occur at the four vertices of $a_{i}, a_{j}$ ). Then either

(1) $j=i+1$ and the annulus $A_{i+1} \in A \Theta(\sigma)$ between $a_{i}, a_{j}$ (or constituent annulus when $\sigma$ has length 3 ) is parallel into $\widehat{F}$; or

(2) $M$ is a Seifert fibered space over $S^{2}$ with three exceptional fibers, one of which has order $m$, and $K$ has bridge number 0 with respect to some genus 2 Heegaard splitting of $M$.

Addendum 5.6. The conclusion above holds when $\sigma$ has length 3 and $K$ intersects $B$ only in $a_{i}, a_{j}$ or in the disk of parallelism of at most one of $a_{i}$ or $a_{j}$.

Proof. We assume $K$ intersects $B$ only at the four vertices of $a_{i}, a_{j}$. Let $A=$ $A_{i+1} \cup A_{i+2} \cdots \cup A_{j}$, where $A_{k} \in A \Theta(\sigma)$ when $\sigma$ is of length 2 , and where $A$ is a constituent annulus of $A \Theta(\sigma)$ between $a_{i}, a_{j}$ when $\sigma$ is of length 3. Let $T=A \cup B$. Note that $A$ and $B$ only intersect in their boundaries (by Lemma 4.14 and the fact 
that any component of Int $A \cap B$ must bound a disk in $A$ ); thus $T$ is an embedded torus in $M$ ( $M$ contains no Klein bottles). Since $M$ is assumed to be atoroidal, $T$ must be compressible in $M$. Let $D$ be a compressing disk.

Addendum. In the context of the Addendum, assume that $K$ intersects $B$ only in the four vertices of $a_{i}, a_{j}$ and in the disk of parallelism of, say, $a_{i}$. Then we may choose the constituent annulus of $A \Theta(\sigma)$ between $a_{i}, a_{j}$ and shrink $B$ so that $A$ and $B$ meet along their boundary and $B$ is disjoint from the interior of the disk of parallelism of $a_{j}$. Then $B$ intersects $K$ only along $a_{i}, a_{j}$, and we apply the arguments to this $A, B$.

The proof of this lemma splits into three cases depending upon the relationship of $A_{i}, A_{j}$, and $D$ with respect to $\widehat{F}$ near $B$.

Case (I). $A_{i+1}$ and $A_{j}$ near $B$ lie on opposite sides of $\widehat{F}$.

The following claim shows that Case (I) does not occur.

Claim 5.7. There is an isotopy of $M$ that reduces the width of $K$ with respect to $\widehat{F}$.

Proof. The unfurling move from section 4.3 of 1 , gives a homeomorphism of $M$ that reduces the width of $K$ and is isotopic to the identity. We describe this below.

Let $V$ be a regular closed neighborhood of $T$ in $M$. Let $\kappa, \kappa^{\prime}$ be the spanning $\operatorname{arcs} K \cap A=K \cap T$ on the annulus $A$. Then $V$ is an interval of tori, $T \times[0,1]$, where we coordinatize $T=S^{1} \times S^{1}$ so that $\partial A=S^{1} \times p_{1} \cup S^{1} \times p_{2}, \kappa \subset q_{1} \times S^{1}$, and $\kappa^{\prime} \subset q_{2} \times S^{1}$ for points $p_{1}, p_{2}, q_{1}, q_{2}$. Now, up to isotopy rel- $\partial$ in $V$, we may take $K \cap V$ to be the pair of $\operatorname{arcs} \widehat{\kappa}, \widehat{\kappa}^{\prime}$ which are $\kappa, \kappa^{\prime}$ extended by product arcs in $V=T \times[0,1]$.

Let $h: V \rightarrow V$ be the homeomorphism that rotates $T \times i$ by $2 \pi i$ in the factor $* \times S^{1}$ and is the identity in the factor $S^{1} \times *$. Under $h, \widehat{\kappa}, \widehat{\kappa^{\prime}}$ become isotopic to spanning arcs of $B$ extended by product $\operatorname{arcs}$ across $V$. As $h$ is the identity on $\partial V$, it extends to $M$ as the identity outside $V$, and the image of $K$ under $h$ is strictly thinner with respect to $\widehat{F}$ (the arcs above and below $\widehat{F}$ after the homeomorphism are level-preserving isotopic to those of $\left.K-\left(\kappa \cup \kappa^{\prime}\right)\right)$. To finish the claim we must show that $h$ is isotopic to the identity on $M$.

As above, let $D$ be a compressing disk for $T$ and $\mathcal{N}=\operatorname{Nbhd}(V \cup D)$. As $M$ is irreducible, the 2-sphere component, $S$, of $\partial \mathcal{N}$ bounds a 3-ball in $M$ which either (a) contains $\mathcal{N}$ or (b) meets it only along $S$.

In case (a), $h$ is the identity on $M$ outside the 3 -ball. So there is an isotopy on the 3-ball, keeping $S$ fixed, taking $h$ restricted to the 3 -ball to the identity. This extends to an isotopy on $M$ taking $h$ to the identity.

In case (b), note that $h$ as a homeomorphism of $V$ is isotopic to the identity by an isotopy that keeps one boundary component of $V$ fixed and rotates the other component of $\partial V$ by $2 \pi$. Take such an isotopy that rotates the component $T_{1}$ of $\partial V$ on the side containing $D$ and fixes the component, $T_{2}$, on the other side. $T_{1}$ bounds a solid torus, $N$, in $M$ that intersects $V$ in $T_{1}$. So we can extend the isotopy of $h$ on $V$ across $N$. As this isotopy fixes $T_{2}$, we may extend it across all of $M$, giving the desired isotopy of $h$ to the identity on $M$.

Case (II). $A_{i+1}$ and $A_{j}$ lie on the same side of $\widehat{F}$ near $B$, and $D$ near $B$ lies on the opposite side of $\widehat{F}$. 
Let $N_{2}$ be the component of $M-T$ containing $D$. By a slight isotopy supported in $\operatorname{Nbhd}(A)$, push $K$ off of $T$ (this comes from looking at the labelling around $a_{i}, a_{j}$ and the fact that $T$ is not a Klein bottle). We may assume that $D$ is disjoint from $K$ since the exterior of $K$ is atoroidal. We will need to handle the cases $m=2$ and $m=3$ separately.

Case (IIa). Assume $m=2$. Then $A^{\prime}=A_{1} \cup \cdots \cup A_{i}$ is an embedded Möbius band that intersects $T$ in only $a_{i}$ by Lemma 4.14. Now $K$ can be perturbed (avoiding $D$ ) so that it is disjoint from $T$ and intersects $A^{\prime}$ in a single point. ( $K$ is made disjoint from $T$ as above. To see that it then intersects the Möbius band $A_{1}$ once, note that when we push $K$ off $A$ it starts off on one side of $A_{i}$ and then ends up on the other.) We may assume that $D$ intersects $A^{\prime}$ in a non-empty collection of arcs which are essential in $A^{\prime}$ (in fact parallel to $K \cap A^{\prime}$ in $A^{\prime}$ by working on the intersections before perturbing $K$ off $A^{\prime}$ ); therefore we get a projective plane or can reduce the number of intersections. Taking an outermost disk of $D-A^{\prime}$ and compressing $A^{\prime}$ along it constructs a compressing disk for $T$ in $N_{2}$ which intersects $K$ exactly once (from the intersection of $K$ with $A^{\prime}$ ). But this contradicts that there is an essential compressing disk for $T$ which misses $K$ in $N_{2}$.

Case (IIb). Assume $m=3$. Then $\Theta^{\prime}=\Theta_{1} \cup \cdots \cup \Theta_{i} \subset A \Theta(\sigma)$ is an embedded twisted $\theta$-band that intersects $T$ in only $a_{i} \subset \theta_{i}$ by Lemma 4.14.

Addendum. In the context of the Addendum in this case, we expand $B$ to include the disks of parallelism. If $K$ intersects the interior of the disk of parallelism of, say, $a_{i}$, we surger this disk along the faces of $\sigma$ connecting the disk of parallelism of $a_{i}$ with that of $a_{j}$ as in the proof of Lemma 5.3. We take the resulting $B$ for the argument below.

Let $\kappa=K \cap \Theta^{\prime}$. Then $D$ intersects $\Theta^{\prime}$ away from $\kappa$. We consider $\Theta^{\prime}-\operatorname{Nbhd}(\kappa)$ as a large trigon, $f$, with corners along $\kappa$ (indeed it is a trigon face in $\Lambda_{x}$ for some label $x$ ). Then we may assume $D$ intersects $f$ only in arcs parallel to the corners of $f$. Furthermore, this collection of arcs is non-empty; otherwise $D$ in union with $\Theta^{\prime}$ along an annulus in $T$ gives rise to a punctured $L(3,1)$ in $M$ by Lemma 4.13. The compression of $\Theta^{\prime}$ along an outermost disk of $D-\Theta^{\prime}$ contains a Möbius band $A^{\prime}$ with $\partial A^{\prime} \subset T$ and $\kappa \subset A^{\prime}$. Then we proceed as in Case (IIa) where $m=2$ to produce a compressing disk for $T$ in $N_{2}$ that intersects $K$ exactly once, contradicting the fact that there is an essential disk disjoint from $K$.

Case (III). $A_{i+1}$ and $A_{j}$ lie on the same side of $\widehat{F}$ near $B$, and $D$ near $B$ lies on this side of $\widehat{F}$.

Since $A_{i+1}$ and $A_{j}$ lie on the same side of $\widehat{F}, i$ and $j$ have different parity.

Let $N_{1}$ be the component of $M-T$ containing $D$. As in Case (II), $K$ may be perturbed to miss $N_{1}$ completely. Note that in the context of the Addendum, the constituent annuli can be taken disjoint from the disks of parallelism of both $a_{i}$ and $a_{j}$.

Compressing $\partial N_{1}$ along $D$ gives a 2-sphere disjoint from $K$. As the exterior of $K$ is irreducible, we see that $N_{1}$ is a solid torus. We show that either $A$ and $B$ are longitudinal annuli in $N_{1}$, that is, that $\Delta\left(\partial D, a_{i}\right)=1$ on $\partial N_{1}$, thereby showing that $A$ is parallel into $B$, or conclusion (2) holds.

If $\Delta\left(\partial D, a_{i}\right)=0$, then $M$ contains a punctured lens space (coming from the elements of $A \Theta(\sigma)$ up to $a_{i}$ in union with $D$ along $\left.a_{i}\right)$. 
Assume $\Delta\left(\partial D, a_{i}\right)=n \geq 2$. Set $V=\operatorname{Nbhd}\left(N_{1} \cup A_{1} \cup \cdots \cup A_{i}\right)$ if $m=2$ or $V=\operatorname{Nbhd}\left(N_{1} \cup \Theta_{1} \cup \ldots \Theta_{i}\right)$ if $m=3$. In the context of the Addendum we expand $B$ to include disks of parallelism of $a_{i}, a_{j}$ and surger away intersects of $K$ with either of these disks of parallelism as in Case (IIb). Then $V$ is a Seifert fibered space over the disk with two exceptional fibers of orders $m$ and $n$ (note $A_{1} \cup \cdots \cup A_{i}$ or $\Theta_{1} \cup \ldots \Theta_{i}$ intersect $T$ only along their boundary; otherwise the core curve of $B$ bounds a disk outside of $N_{1}$, which along with $N_{1}$ would create a lens space summand in $M$ ).

$K$ can be perturbed so that it is disjoint from $N_{1}$ and intersects $A_{1} \cup \cdots \cup A_{i}$ or $\Theta_{1} \cup \cdots \cup \Theta_{i}$ in a single point if $m=2$ or $m=3$ respectively (this is most easily seen by checking the labellings around the vertices of $a_{i}$; see Figure 2). Thus $K \cap V$ is isotopic rel boundary to the co-core of the unique essential annulus in the Seifert fibered space, $V$. It follows from this that the twice-punctured torus $S=\partial V-\operatorname{Nbhd}(K)$ is incompressible in $V-\operatorname{Nbhd}(K)$.

Claim 5.8. $K \cap(M-V)$ is isotopic rel boundary in $M-V$ onto $\partial V$.

Proof. Let $R=\partial(M-V-\operatorname{Nbhd}(K))$. Then $R$ must be compressible in the exterior of $K, X$, by Theorem 2.4.3 of [7] (where $R$ is the $S$ there and the $r_{0}$ there is $p / q$ ). As $S$ is incompressible in $V$ - Nbhd $K, R$ must compress in $M-V-$ Nbhd $K$. If $S$ were incompressible in $M-V-$ Nbhd $K$, then the Handle Addition Lemma, Lemma 2.1.1 of [7], would imply that $\partial V$ is incompressible in $M-V$. But this contradicts that $M$ is atoroidal. Thus $S$ must compress in $M-V-$ Nbhd $K$. As $X$ is anannular, $S$ must compress in $M-V$ to a boundary parallel annulus. This implies that $K \cap(M-V)$ is isotopic in $M-V$ to $\partial V$, as claimed.

Since $M$ is atoroidal, and $\partial V$ is incompressible in $V, \partial V$ must compress outside of $V$. Compressing $\partial V$ then yields a 2 -sphere that bounds a $3-$ ball $B^{3}$. If this 3 -ball contained $V$, then $K$ could be isotoped into this 3-ball, contradicting the irreducibility of the exterior of $K$. So we may assume that this 3-ball lies outside of $V$, and consequently that $M-V$ is a solid torus. Then $M$ is a Seifert-fibered space over the 2-sphere with three exceptional fibers one of which has order $m$. Let $h=\operatorname{Nbhd}(K \cap V)$. One checks that $H_{1}=V-h$ is a genus two handlebody. Certainly, $H_{2}=(M-V) \cup h$ is a genus two handlebody. That is, $H_{1} \cup H_{2}$ is a Heegaard splitting of $M$. Recall that $K \cap(M-V)$ can be isotoped rel its boundary onto $\partial V$. Thus $K$ can be isotoped onto $\partial H_{1}$. This is conclusion (2) of the Lemma.

Thus we may assume $\Delta\left(\partial D, a_{i}\right)=1$ and $N_{1}$ gives an isotopy of $A$ to $B$ as desired. If $j>i+1$, then this isotopy from $A$ to $B$ gives a thinner presentation of $K$ (the two sub-arcs $K \cap A$ of $K$ are flattened onto $\widehat{F}$ by this isotopy; perturb them back slightly off of $\widehat{F}$ to reinstate a Morse presentation of $K$ with fewer extrema.) As we started with a thin presentation of $K$, it must be that $j=i+1$.

These 3 cases exhaust the possibilities, thereby completing the proof of Lemma 5.5.

Lemma 5.9. Let $\sigma$ be an extended Scharlemann cycle of length $m=2$ or $m=3$. Assume that conclusion (2) of Lemma 5.5 does not hold. If $a_{i}, a_{j}, a_{k} \in a \theta(\sigma)$ together lie on an annulus in $\widehat{F}$, then there must be intersections of $K$ with this annulus that do not belong to $a_{i}, a_{j}, a_{k}$.

Proof. Without loss of generality assume $a_{i}, a_{j}$ cobound annulus $B_{1}$ on $\widehat{F}$ and $a_{j}, a_{k}$ cobound annulus $B_{2}$ on $\widehat{F}$, such that $K$ intersects $B_{1} \cup B_{2}$ only along $a_{i}, a_{j}, a_{k}$ and 
such that $B_{1}, B_{2}$ have disjoint interiors. Then by Lemma 5.5 we may assume $j=i+1, k=i+2$ and the annuli or constituent annuli, $A_{i}, A_{i+1}$, of $A \Theta(\sigma)$ are parallel into $B_{1}, B_{2} \subset \widehat{F}$. But then we can use these parallelisms of the annuli to guide a thinning of $K$ with respect to $\widehat{F}$.

Lemma 5.10. Let $\sigma$ be an extended Scharlemann cycle of length $m=3$. Assume that conclusion (2) of Lemma 5.5 does not hold. Assume that $\theta$-curves $a_{i}, a_{j}, a_{k} \in$ $a \theta(\sigma)$ together lie on an annulus in $\widehat{F}$, with $a_{j}$ between $a_{i}, a_{k}$ on $\widehat{F}$. Then there must be intersections of $K$ with this annulus that do not belong to $a_{i}, a_{j}, a_{k}$ or to the disks of parallelism of $a_{i}, a_{k}$.

Proof. Assume the only other intersections of $K$ occur within the disks of parallelism of $a_{i}, a_{k}$. We apply the proof of Lemma 5.9] using the Addendum to Lemma 5.5.

\subsection{Parallelisms of $a(\sigma)$ and $\theta(\tau)$.}

Definition 5.11. An extended Scharlemann cycle is called proper if in its corner no label appears twice.

Lemma 5.12. Assume that $\widehat{F}$ is a strongly irreducible Heegaard surface for $M$. Let $\sigma, \tau$ be proper extended Scharlemann cycles of $\Lambda$ of length 2 or 3 with different core labels. If there is an annulus, $A$, in $\widehat{F}$ containing an element of $a \theta(\sigma)$ and an element of $a \theta(\tau)$, then either

(1) $L(\sigma)$ and $L(\tau)$ intersect in two non-empty label intervals, $L(\sigma) \cup L(\tau)$ includes all labels, and one of $\sigma$ or $\tau$ has length 3 ; or

(2) $M$ is a Seifert fibered space over the 2-sphere with three exceptional fibers, and the orders of two of these exceptional fibers are the lengths of $\sigma, \tau$. Furthermore, there is a genus 2 (hence minimal genus) Heegaard splitting of $M$ with respect to which $K$ has bridge number at most $k-1$, where $k=|A \cap K|$.

Proof. After possibly paring down $\sigma, \tau$ we may assume that $a \theta_{\sigma}=\partial(A \Theta(\sigma)), a \theta_{\tau}=$ $\partial(A \Theta(\tau))$ are the only elements of $a \theta(\sigma), a \theta(\tau)$ (resp.) lying entirely in $A$. If there are intersections of $A \Theta(\sigma), A \Theta(\tau)$ with $A$ coming from the interior of faces of $\sigma, \tau$, then we may assume they are essential simple closed curves in $A$ (Lemma 2.21). Take such a curve that is innermost on $\sigma$, say, bounding a disk $D$. Surgery of $A$ along $D$ gives a disk $A^{\prime}$ which we may take (after possibly further surgeries) to intersect $A \Theta(\sigma)$ only along its boundary. But then $\operatorname{Nbhd}\left(A \Theta(\sigma) \cup A^{\prime}\right)$ is a lens space summand in $M$ - a contradiction. Thus we may assume that $\sigma, \tau$ intersect $A$ only along their edges. The argument now divides up according to $L(\sigma) \cap L(\tau)$. See Lemma 4.2 for the possibilities. We work through these, ending with Case IV that $L(\sigma) \cup L(\tau)$ contains all labels and $L(\sigma) \cap L(\tau) \neq \emptyset$. In this final case we show that at least one of $\sigma, \tau$ must be of length 3, giving conclusion (1) above. The generic argument is Case I.

Case I. $L(\sigma) \cap L(\tau)=\emptyset$.

Proof of Case I. Then $A \Theta(\sigma) \cap A \Theta(\tau)=\emptyset$ and we may assume $A \Theta(\sigma), A \Theta(\tau)$ intersect $A$ only along their boundaries. Consequently $N=\operatorname{Nbhd}(A \Theta(\sigma) \cup A \cup$ $A \Theta(\tau))$ is a Seifert fibered space over the disk with two exceptional fibers whose orders are given by the lengths of $\sigma, \tau$ (e.g. Lemma 4.13). Note that as $M$ contains no Klein bottles, the length of either $\sigma$ or $\tau$ must be 3 . 


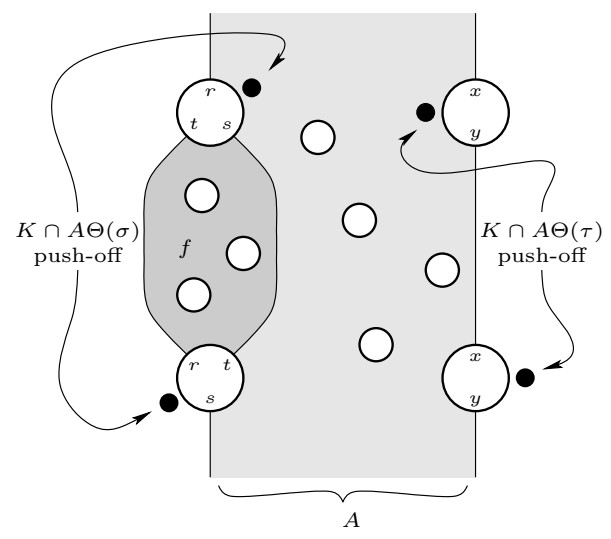

FIGURE 2

Claim 5.13. $M$ is a Seifert fibered space over the 2-sphere with three exceptional fibers, where the orders of the exceptional fibers include the lengths of $\sigma$ and $\tau$. Furthermore, $p / q$ is not a boundary slope for $X$.

Proof. As $M$ is atoroidal, $\partial N$ compresses in $M-N$. As $M$ is irreducible, the resulting 2-sphere bounds a 3 -ball.

First assume this 3-ball contains $N$. Then the core of $A$ is an essential curve in $\widehat{F}$ that does not bound a meridian on either side of $\widehat{F}$ (otherwise, as argued above, it in union with $A \Theta(\sigma)$ can be surgered to produce a lens space summand in $M)$. Then Lemma 3.3 of [3] (which shows if a Heegaard surface of a 3-manifold other than $S^{3}$ contains a non-meridional simple closed curve that lies in a 3 -ball, then the splitting is weakly reducible) contradicts our assumption that $\widehat{F}$ is strongly irreducible.

Thus the 3-ball is disjoint from $\partial N$. That is $M-N$ is a solid torus. Then $M$ is a Seifert fibered space over the two sphere with three exceptional fibers with finite first homology. Furthermore, $M$ contains no incompressible surface. Thus Theorem 2.0.3 of [7] implies that if $p / q$ were a boundary slope, then $X(r)$ would contain an incompressible surface whenever $\Delta(r, p / q)>1$. Since $q \geq 2$ and $X(1 / 0)$ is $S^{3}$, this is impossible.

Claim 5.14. $K \cap N$ consists of at most $k-2$ arcs which can be isotoped (rel $\partial N$ ) to a collection of co-cores of the essential annulus in the Seifert fibered space $N$.

Proof. First note that each of $K \cap A \Theta(\sigma), K \cap A \Theta(\tau)$ can be isotoped off of $A \Theta(\sigma), A \Theta(\tau)$ (resp.) to intersect $A$ in a single point. See Figure 2. Thus we may take $K \cap N$ to be a collection of $k-2$ arcs of the form point $\times I \subset A \times I$. Each of these arcs can be isotoped to a co-core of the essential annulus $c \times I \subset N$, where $c$ is the core of $A$ : In particular, say $\sigma$ is a length 3 extended Scharlemann cycle. Let $f$ be the disk of parallelism in $G_{F}$ between two edges of $\partial \Theta(\sigma)$ (as shown in Figure 2). Then the arc $p \times I \subset f \times I$, where $p$ is a point of $f$, can be isotoped, rel $\partial N$, across $\operatorname{Nbhd}(\Theta(\sigma))$ to a co-core of $c \times I$ in $N$. Figure 3 shows an isotopy of $K$ moving an intersection with $f$ out to an edge of $\partial f$ and then through the trigon face of $\sigma$ to produce an intersection with $A-f$; the dashed circles show the resulting new intersections $K \cap \widehat{F}$. 

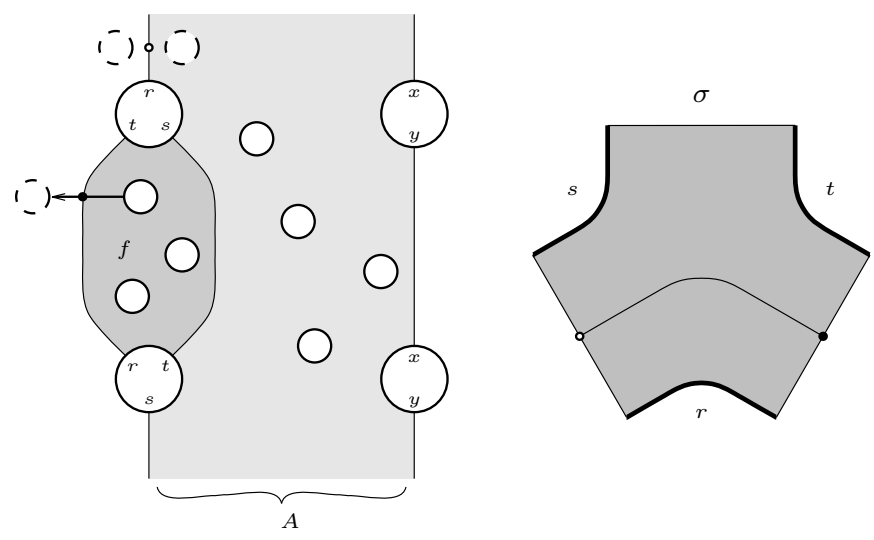

Figure 3

$\partial N \subset M$ is a 2-torus. Let $T=\partial N-\operatorname{Nbhd}(K)$. Maximally compress $T$ in $X$. Claim 5.13 says that $p / q$ is not a $\partial$-slope, thus $T$ compresses to $\partial$-parallel annuli in $X$. Note that these annuli may be nested in $X$. Each $\partial$-parallel annulus defines an isotopy in $M$ of a subarc of $K$, with endpoints in $K \cap \partial N$, onto $\partial N$ - keeping the endpoints of the arc fixed. Furthermore, every point of $K \cap \partial N$ belongs to such an arc of $K$. Let $k_{1}^{\prime}, \ldots, k_{r}^{\prime}$ be the subarcs of $K$ lying within the outermost of these $\partial$-parallel annuli and let $k_{1}, \ldots, k_{r}$ be the complementary subarcs of $K$. Note that the $k_{i}$ lie on the same side of $\partial N$. Then the isotopies described above corresponding to the outermost annuli deform $K$ to the union of the $\operatorname{arcs} k_{1}, \ldots, k_{r}$ along with arcs that lie in $\partial N$. As the complement of $K$ in $M$ is hyperbolic, it must be that the $\operatorname{arcs} k_{1}, \ldots, k_{r}$ lie in $N$. By the claim above, $k_{1}, \ldots, k_{r}$ can be isotoped to co-cores of the essential annulus in $N$. Let $\alpha$ be a disjoint co-core of this annulus. Then $N-\operatorname{Nbhd}(\alpha),(M-N) \cup \operatorname{Nbhd}(\alpha)$ is a genus 2 splitting of $M$. Furthermore, each of $k_{1}, \ldots, k_{r}$ is $\partial$-parallel in $N-\operatorname{Nbhd}(\alpha)$. Perturbing each arc of $K-\left(\cup k_{i}\right)$ into $M-N$ puts $K$ in bridge position with respect to this genus 2 splitting. Noting that $r \leq k-2$, we have conclusion (2) of Lemma 5.12 .

In the remaining cases, the argument of Case I gives the desired conclusion once we show how to modify $A \Theta(\sigma)$ and $A \Theta(\tau)$ so that they are mutually disjoint and intersect $A$ only along their boundaries, $a \theta_{\sigma}, a \theta_{\tau}$. We write $A \Theta(\sigma)=E_{\sigma} \cup$ $F_{\sigma}, A \Theta(\tau)=E_{\tau} \cup F_{\tau}$, where $F_{\sigma}, F_{\tau}$ is the union of faces of $\sigma, \tau$ (resp.) (thought of as disks in $X$ ), and where $E_{\sigma}, E_{\tau}$ are rectangles in $\operatorname{Nbhd}(K)$ describing an extension of $F_{\sigma}, F_{\tau}$ across $\operatorname{Nbhd}(K)$ to form the long Möbius band or twisted $\theta$-band. In what follows (at least up to Case IV') we show how to choose $E_{\sigma}, E_{\tau}$ to be disjoint, making $A \Theta(\sigma), A \Theta(\tau)$ disjoint and intersecting $A$ along $\partial A$.

Let $\{x, z\}$ be the extremal labels of $\sigma$, and $\{y, w\}$ the extremal labels of $\tau$. Let $\{\alpha, \beta\}$ or $\{\alpha, \beta, \gamma\}$ be the corners of $\sigma$ depending on the length of $\sigma$. Similarly let $\left\{\alpha^{\prime}, \beta^{\prime}\right\}$ or $\left\{\alpha^{\prime}, \beta^{\prime}, \gamma^{\prime}\right\}$ be the corners of $\tau$ according to its length.

Case II. $L(\sigma) \cap L(\tau)$ is a single interval of labels $\overline{x y}$ (including a point interval).

Proof. First we consider the case of a point interval, that is, when $x=y$ (and $z \neq w$ ). Then $a \theta_{\sigma}, a \theta_{\tau}$ intersect in a single point (at vertex $x=y$ ). As $a \theta_{\sigma}, a \theta_{\tau}$ both lie in $A$, they must be non-transverse around vertex $x$ on $G_{F}$. This means 


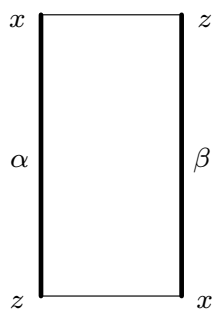

$\sigma$

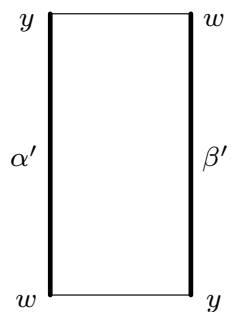

$\tau$

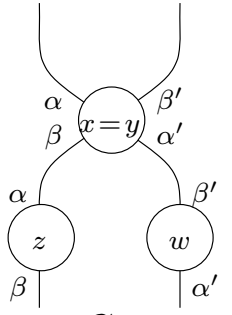

$G_{F}$

FiguRE 4

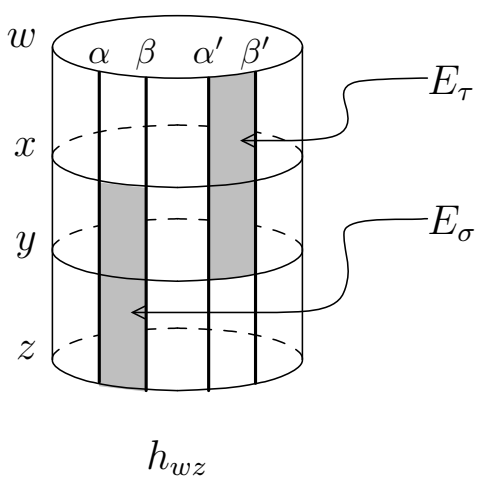

FIGURE 5

that as one reads around vertex $x$ on $G_{F}$, labels $\{\alpha, \beta\}(\{\alpha, \beta, \gamma\})$ do not separate the labels $\left\{\alpha^{\prime}, \beta^{\prime}\right\}\left(\left\{\alpha^{\prime}, \beta^{\prime}, \gamma^{\prime}\right\}\right)$. See Figure 4 for $\sigma, \tau$ of length 2 . We choose disjoint $E_{\sigma}, E_{\tau}$ as pictured in Figure 5 (with $x=y$ ), making $A \Theta(\sigma), A \Theta(\tau)$ disjoint. The argument for Case I now gives a genus 2 splitting of $M$ with bridge number at most $k-1$ ( $K$ intersects $A$ along $a \theta_{\sigma}, a \theta_{\tau}$ only 3 times here, rather than 4 ).

Thus we may assume that $\{x, z\} \cap\{y, w\}=\emptyset$. Let $b_{\sigma}$ be the component of $a \theta(\sigma)$ through vertex $y$. Then $b_{\sigma}$ intersects $a \theta_{\tau}$ in a single point (at $y$ ). See Figures 6 , 7. Again $b_{\sigma}$ is disjoint from $a \theta_{\sigma}$ which lies in $A$ with $a \theta_{\tau}$, so $b_{\sigma}$ must intersect $a \theta_{\tau}$ tangentially. Thus, as one transverses vertex $y$ in $G_{F}$ the $\{\alpha, \beta\}(\{\alpha, \beta, \gamma\})$ labels do not separate $\left\{\alpha^{\prime}, \beta^{\prime}\right\}\left(\left\{\alpha^{\prime}, \beta^{\prime}, \gamma^{\prime}\right\}\right)$. We then may choose disjoint $E_{\sigma}, E_{\tau}$ as pictured in Figures 5,8 . The argument of Case I now gives a genus 2 splitting of $M$ with bridge number at most $k-2$.

Case III. $L(\tau) \subset L(\sigma)$ or $L(\sigma) \subset L(\tau)$.

Proof. The argument is the same in either case. We assume $L(\tau) \subset L(\sigma)$.

We may assume that, say, $y \neq x, z$. Let $b_{\sigma}$ be the component of $a \theta(\sigma)$ through vertex $y$ - connecting $y$ to another vertex $r$. If $r=w$, then $\sigma, \tau$ have the same core labels, contrary to our assumption.

Thus we assume $r \neq y, w, x, z\left(r \neq y\right.$ by the Parity Rule). Then $b_{\sigma}$ intersects $a \theta_{\tau}$ in a single point (at the vertex $y$ ). Since $b_{\sigma}$ is disjoint from $a \theta_{\sigma}$, and $a \theta_{\sigma}, a \theta_{\tau}$ are contained in $A, b_{\sigma}$ must intersect $a \theta_{\tau}$ tangentially. That is, as one transverses around the (fat) vertex $y$ of $G_{F}$ the labels $\{\alpha, \beta\}(\{\alpha, \beta, \gamma\})$ are not separated by 

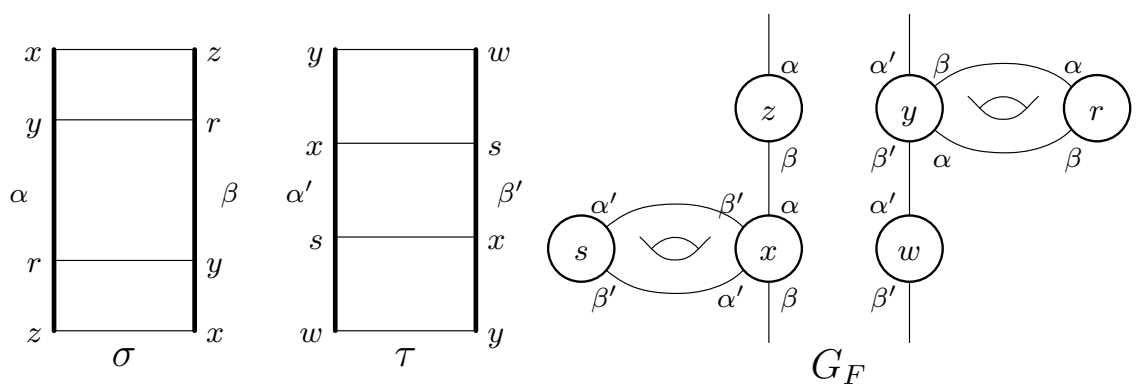

FiguRE 6

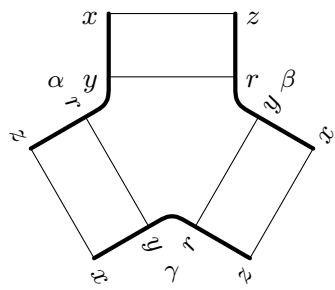

$\sigma$

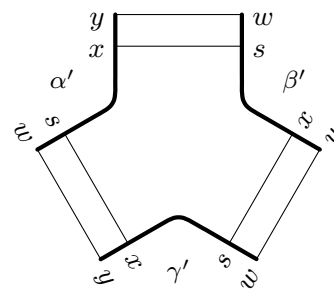

$\tau$

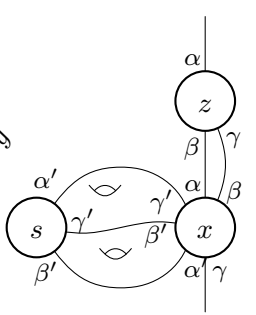

$G_{F}$

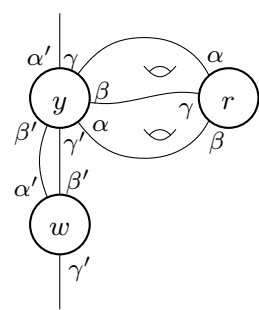

FIGURE 7

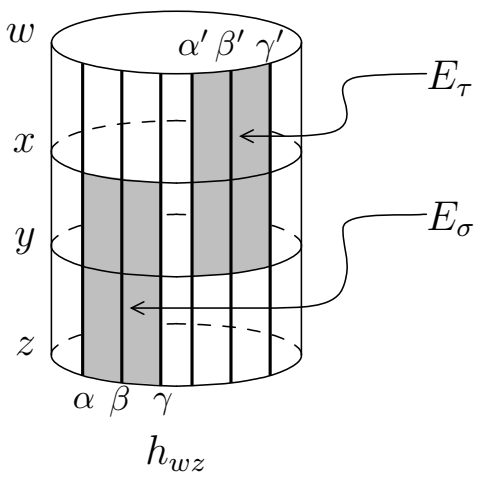

Figure 8

the labels $\left\{\alpha^{\prime}, \beta^{\prime}\right\}\left(\left\{\alpha^{\prime}, \beta^{\prime}, \gamma^{\prime}\right\}\right)$. Thus in $\operatorname{Nbhd}(K)$, we may choose disjoint $E_{\sigma}, E_{\tau}$ as pictured in Figure 9 (which illustrates the case of length 2 with $r$ between $y$ and $w)$, thereby making $A \Theta(\sigma), A \Theta(\tau)$ disjoint. We now apply the argument from Case I giving a $k-2$ bridge presentation of $K$.

Case IV. $L(\sigma) \cup L(\tau)$ contains all labels of $G_{Q}$, and $L(\sigma)$ overlaps $L(\tau)$ in two intervals of labels: $\overline{x y}$ and $\overline{w z}$.

This case is conclusion (1) of the lemma once we have shown that one of $\sigma, \tau$ is of length 3. So we assume that $\sigma, \tau$ are both of length 2 and we are as in Figure 10 . 


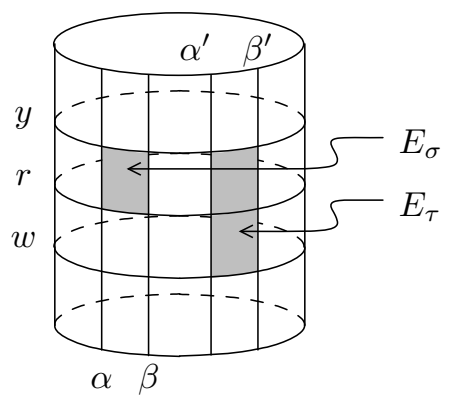

Figure 9
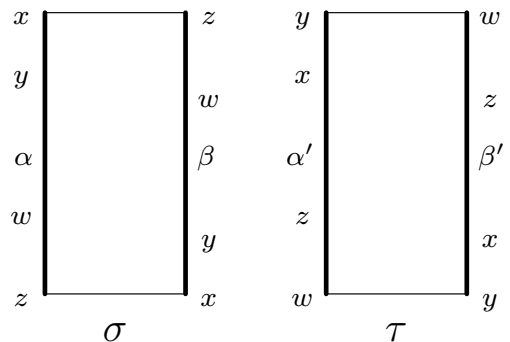

FIGURE 10

If $\{x, z\}=\{y, w\}$, then $a_{\sigma}=\partial A(\sigma), a_{\tau}=\partial A(\tau)$ are isotopic on $\widehat{F}$ and both go through vertices $x, z$ of $\widehat{F}$. Thus $A(\sigma), A(\tau)$ can be amalgamated along their boundary to create an embedded Klein bottle.

Next assume that $x=y$ but $z \neq w$. Let $b_{\sigma}$ be the component of $a(\sigma)$ through vertex $w$. As $b_{\sigma}$ is disjoint from $a_{\sigma}$ and intersects $a_{\tau}$ once at $w, b_{\sigma}$ and $a_{\tau}$ intersect non-transversely. Thus around vertex $w$, the labels $\{\alpha, \beta\}$ do not separate $\left\{\alpha^{\prime}, \beta^{\prime}\right\}$. Similarly, as $a_{\sigma}, a_{\tau}$ intersect in a single point at vertex $x$ and yet are isotopic, their intersection is non-transverse. That is, around vertex $x$, the labels $\{\alpha, \beta\}$ do not separate $\left\{\alpha^{\prime}, \beta^{\prime}\right\}$. Figure 11 (with $x=y$ ) shows that we can choose disjoint $E_{\sigma}, E_{\tau}$.

Thus we may assume $\{x, z\} \cap\{y, w\}=\emptyset$. Let $b_{\sigma}$ be the component of $a(\sigma)$ through vertex $y$, and let $r$ be the other vertex of $G_{F}$ to which $b_{\sigma}$ is incident. Then $r \neq x, z$.

Assume $r \neq w$. Then as $b_{\sigma}$ intersects $a_{\tau}$ once and is disjoint from $a_{\sigma}$, it must intersect $a_{\tau}$ non-transversely. That is, around vertex $y$ the labels $\{\alpha, \beta\}$ do not separate $\left\{\alpha^{\prime}, \beta^{\prime}\right\}$. Let $c_{\sigma}$ be the component of $a(\sigma)$ through vertex $w$. Again, $c_{\sigma}$ must intersect $a_{\tau}$ non-transversely at $w$. Hence around $w$ in $G_{F}$, the labels $\{\alpha, \beta\}$ do not separate $\left\{\alpha^{\prime}, \beta^{\prime}\right\}$. Thus we may choose disjoint disks $E_{\sigma}, E_{\tau}$ in $\operatorname{Nbhd}(K)$ as pictured in Figure 11,

This leaves us with the case that $r=w$, whose argument is slightly different from the preceding ones.

Case IV'. In Case IV above $r=w$.

Proof. This is the case when the core labels of $\sigma, \tau$ are "antipodal" labels. Let $b_{\sigma}$ be the component of $a(\sigma)$ through vertices $y$ and $w$ of $G_{F}$. Then $b_{\sigma}$ and $a_{\tau}$ intersect twice. Since $b_{\sigma}$ is disjoint from $a_{\sigma}$ which is isotopic to $a_{\tau}$, the algebraic 


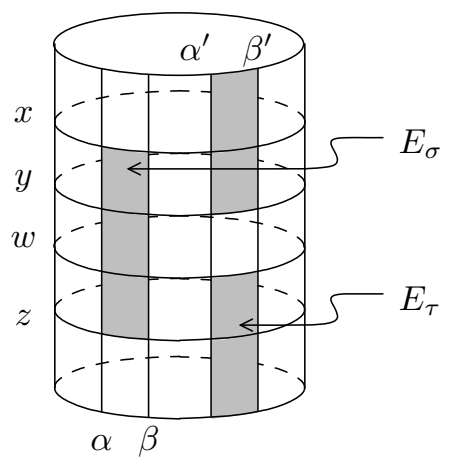

FiguRE 11

intersection number of $b_{\sigma}$ and $a_{\tau}$ is 0 . Thus we can choose $E_{\sigma}, E_{\tau}$ in $\operatorname{Nbhd}(K)$ so that they are either (1) disjoint as in Figure 11 or (2) intersect in exactly two arcs as in Figure 12. This follows since the labels $\{\alpha, \beta\}$ must separate $\left\{\alpha^{\prime}, \beta^{\prime}\right\}$ either (1) around neither vertices $y, w$ or (2) around both vertices $y, w$.

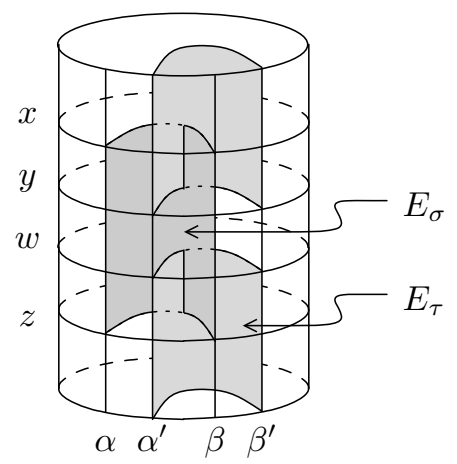

FIGURE 12

$A$ is the annulus on $\widehat{F}$ between $a_{\sigma}$ and $a_{\tau}$. We may assume $\operatorname{Int} A$ is disjoint from the vertices of $G_{F}$ (otherwise there is another component of $a(\sigma)$ or $a(\tau)$ in $A$, which we initially assumed is not the case). Consider $A(\sigma)=E_{\sigma} \cup F_{\sigma}, A(\tau)=E_{\tau} \cup F_{\tau}$, where $F_{\sigma}, F_{\tau}$ is the union of faces of $\sigma, \tau$. Then $A(\sigma), A(\tau)$ are either (1) disjoint or (2) intersect in two double arcs (from $x$ to $y$ and $w$ to $z$ along $K$ ). If (1), $M$ contains an embedded Klein bottle. If (2), $S=A(\sigma) \cup A \cup A(\tau)$ is a Klein bottle that self-intersects in a single double-curve (again, we may assume $A(\sigma), A(\tau)$ are disjoint from $A$ except along $a(\sigma), a(\tau))$. The two preimage curves are disjoint from the cores of each of $A(\sigma), A(\tau)$, and $A$, and consequently bound disjoint disks, Möbius bands in the preimage. We may surger along the double curve to obtain an embedded projective plane or Klein bottle in $M$.

This completes the proof of Lemma 5.12

Corollary 5.15. Let $\sigma, \tau$ be proper extended Scharlemann cycles of $\Lambda$ of length 2 . If there is an annulus, $A$, in $\widehat{F}$ containing a component of $a(\sigma)$ and a component of $a(\tau)$, then $\sigma$ and $\tau$ have the same core labels. 
Proof. In this context, the proof of Lemma 5.12 constructs within $M$ a Seifert fibered space over the disk with two exceptional fibers each of order 2. But then $M$ would contain a Klein bottle, a contradiction.

Lemma 5.16. Assume $\widehat{F}$ is a strongly irreducible Heegaard surface for $M$. If $\sigma, \tau$ are extended Scharlemann cycles of $\Lambda$ of length 2 or 3 with the property that $L(\sigma) \cup L(\tau)$ includes all labels, then either

(1) $t \leq 32(g-1)$; or

(2) $M$ is a Seifert fibered space over $S^{2}$ with three exceptional fibers, where at least one of the exceptional fibers has order 2 or 3 . Furthermore, there is a genus 2 Heegaard splitting of $M$ with respect to which $K$ is at most 4-bridge.

Proof. We may assume, by paring down as needed, that $\sigma$ and $\tau$ are proper. We may also assume $|L(\sigma)| \geq|L(\tau)|$; hence $|L(\sigma)| \geq t / 2$. If (1) does not hold, then we have $|a(\theta(\sigma))|=|L(\sigma)| / 2 \geq t / 4>8(g-1)$. Note that the elements of $a \theta(\sigma)$ are disjoint.

Since a $\theta$-curve has Euler characteristic -1, the maximal number of disjoint $\theta$-curves on $\widehat{F}$, none of which lies in an annulus, is $-\chi(\widehat{F})=2(g-1)$. Suppose $\sigma$ has length 3. Since $|a \theta(\sigma)|>8(g-1)$, there must be more than $6(g-1)$ elements of $a \theta(\sigma)$ that lie in annuli, and hence at least three such elements that are isotopic on $\widehat{F}$ and contain no further elements of $a \theta(\sigma)$ between them. The same holds $a$ fortiori if $\sigma$ has length 2 .

By Lemma 5.9, either conclusion (2) of Lemma 5.16 holds, or there must be intersections of $K$ with an annulus in $\widehat{F}$ between a pair of these elements, $c_{1}, c_{2}$. We assume the latter. Such an intersection gives rise to a vertex of $G_{F}$ that must correspond to an element of $L(\tau)$ that is not in $L(\sigma)$. Thus there is an element, $b$, of $a \theta(\tau)$ lying in the annulus of $\widehat{F}$ cobounded by $c_{1}, c_{2}$. Then $b$ must be parallel to and disjoint from, say, $c_{1}$. Taking $b$ to be the nearest such to $c_{1}$, we may assume $c_{1}, b$ cobound an annulus $A \subset \widehat{F}$ such that $|K \cap A| \leq 5$ (four from the vertices of $c_{1}, b$ and at most one more in the interior of $A$ belonging to an element of $a \theta(\tau)$ whose other vertex belongs to $\left.c_{1}\right)$. Now pare down $\sigma, \tau$ so that $c_{1}=\partial A \Theta(\sigma)$ and $b=\partial A \Theta(\tau)$. Then, as $b$ contains a vertex which is not in $L(\sigma), L(\sigma)$ and $L(\tau)$ can intersect in at most one label interval. Now Lemma 5.12 gives conclusion (2) above.

\section{TRIgons of Type I OR II}

Lemma 6.1. Let $\sigma$ be a trigon face of $\Lambda_{x}$ which is not an extended Scharlemann cycle. Then $\sigma$ must contain a length 2 Scharlemann cycle in its interior.

In particular, assume $\sigma$ is not an $x$-edge cycle. That is, one corner, $\beta$, of $\sigma$ has no $x$ labels, and another corner, $\gamma$, has two. Then there is a length 2 extended Scharlemann cycle in $\sigma$ on the arm opposite $\beta$. Furthermore, if $\sigma$ has a second Scharlemann cycle in its interior, it must lie on the arm of $\sigma$ between $\beta$ and $\gamma$.

Proof. Assume that $\sigma$ is an $x$-edge cycle. Then we may assume it is as in Figure 13, where $j, k$ and $l$ are not all equal. If there is no order 2 Scharlemann cycle in $\sigma$, then we must have $k<j+1, \ell<k+1$, and $j<\ell+1$. All three inequalities cannot simultaneously be true. 

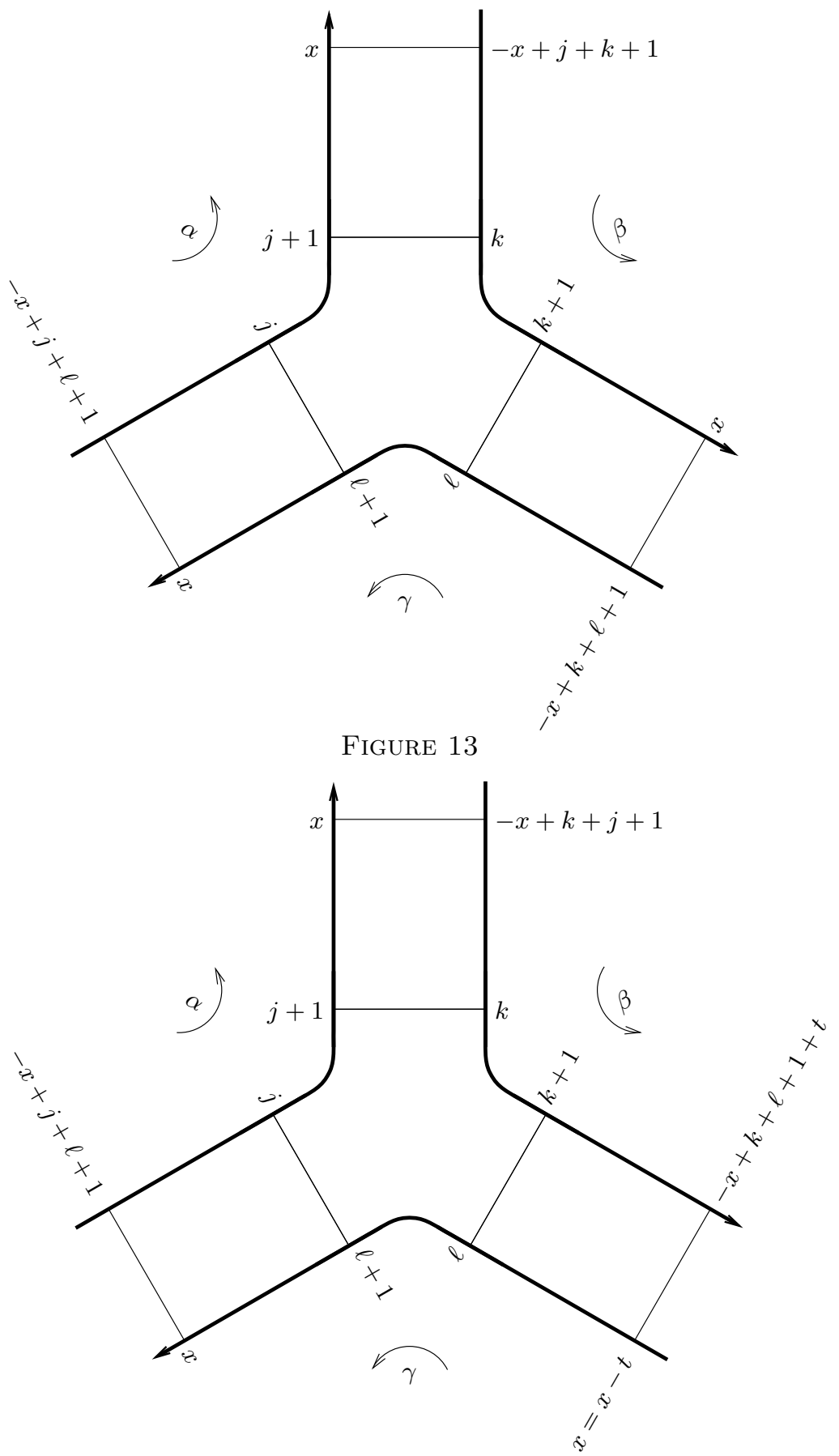

Figure 14 


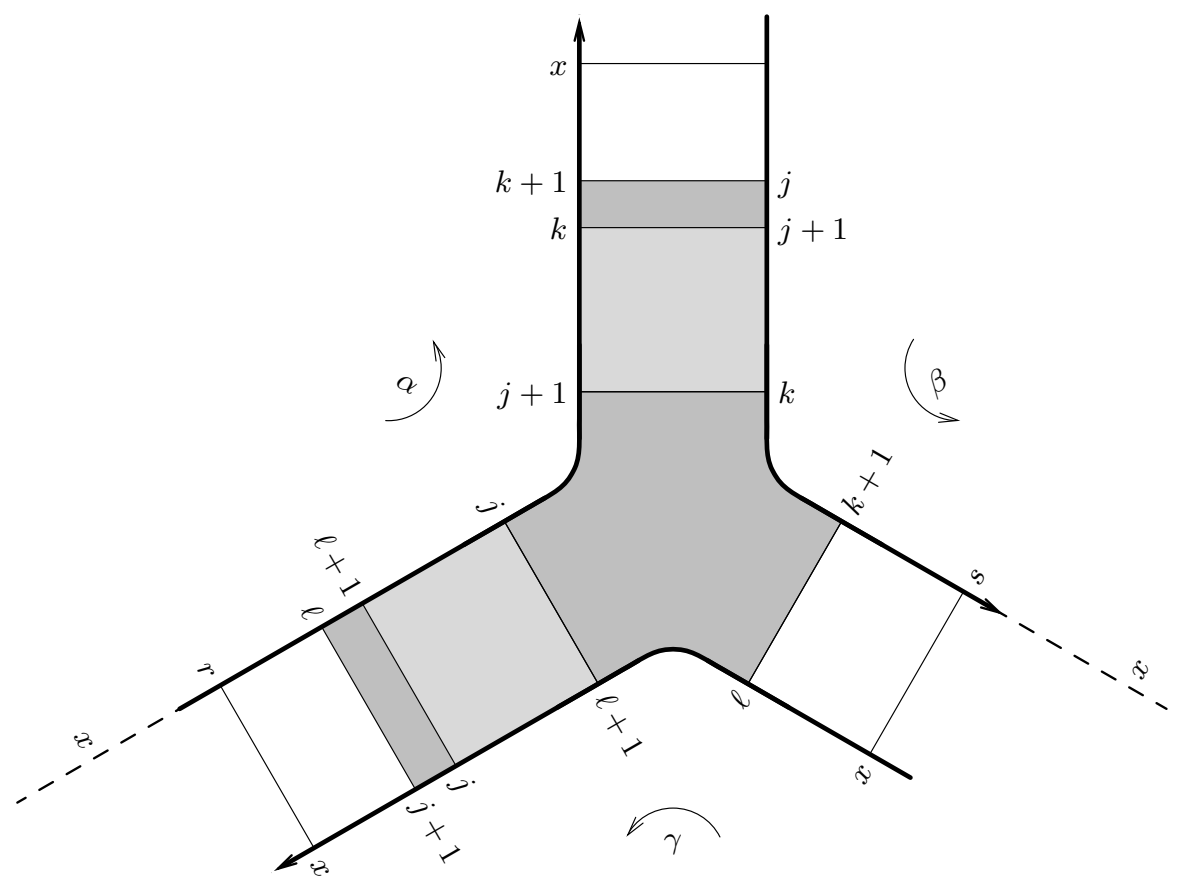

FIGURE 15

Now assume $\sigma$ is not an $x$-edge cycle. We may take it as in Figure 14 Assume there is no Scharlemann cycle in the arm of $\sigma$ opposite to $\beta$. Then $j$ cannot be in $\overline{\ell+1, x}$ on $\gamma$. Thus $\ell+1$ must be in $\overline{j+1, x}$ on $\alpha$. Hence the length of the $\beta$-corner must be at least as large as that of $\gamma$ and $\beta$ would contain a label $x$.

Now assume that $\sigma$ contains a second Scharlemann cycle in its $\alpha \beta$-arm. Refer to Figure 15, Looking at the $\alpha \gamma$-arm, we see that the interval $\overline{x \ell}$ on the extension of $\alpha$ must be at least as large as $\overline{j x}$ on $\gamma$, which in turn is strictly larger than $\overline{k x}$ on $\alpha$. But $\overline{k x}$ on $\alpha$ must be larger than $\overline{k s}$ on $\beta$. Looking at the $\gamma \beta$-arm, though, we see that $\overline{k s}$ on $\beta$ is larger than $\overline{x \ell}$ on $\gamma-$ a contradiction.

Definition 6.2. Let $\sigma$ be a trigon face of $\Lambda_{x}$, considered as a subgraph of $\Lambda$, that does not correspond to an extended Scharlemann cycle of length 3. By Lemma 6.1. $\sigma$ must contain a Scharlemann cycle of length 2. As $\sigma$ is a face of $\Lambda_{x}$ any such Scharlemann cycle must be the core of an extended Scharlemann cycle in $\sigma$ that abuts the central trigon of $\sigma$ and is disjoint from any $x$-edge of $\sigma$ (i.e. the extended Scharlemann cycle lies within $\sigma$ abutting the central trigon). There can be at most two such Scharlemann cycles within $\sigma$, which must lie in different arms of $\sigma$. We say that $\sigma$ is a Type I trigon if there is only one and the corners of its central trigon are on three different pairs of labels. It is of Type II if it contains two such Scharlemann cycles. It is a Type III trigon otherwise - that is, it contains a single length 2 Scharlemann cycle and the three corners in its central trigon represent two different label pairs. 

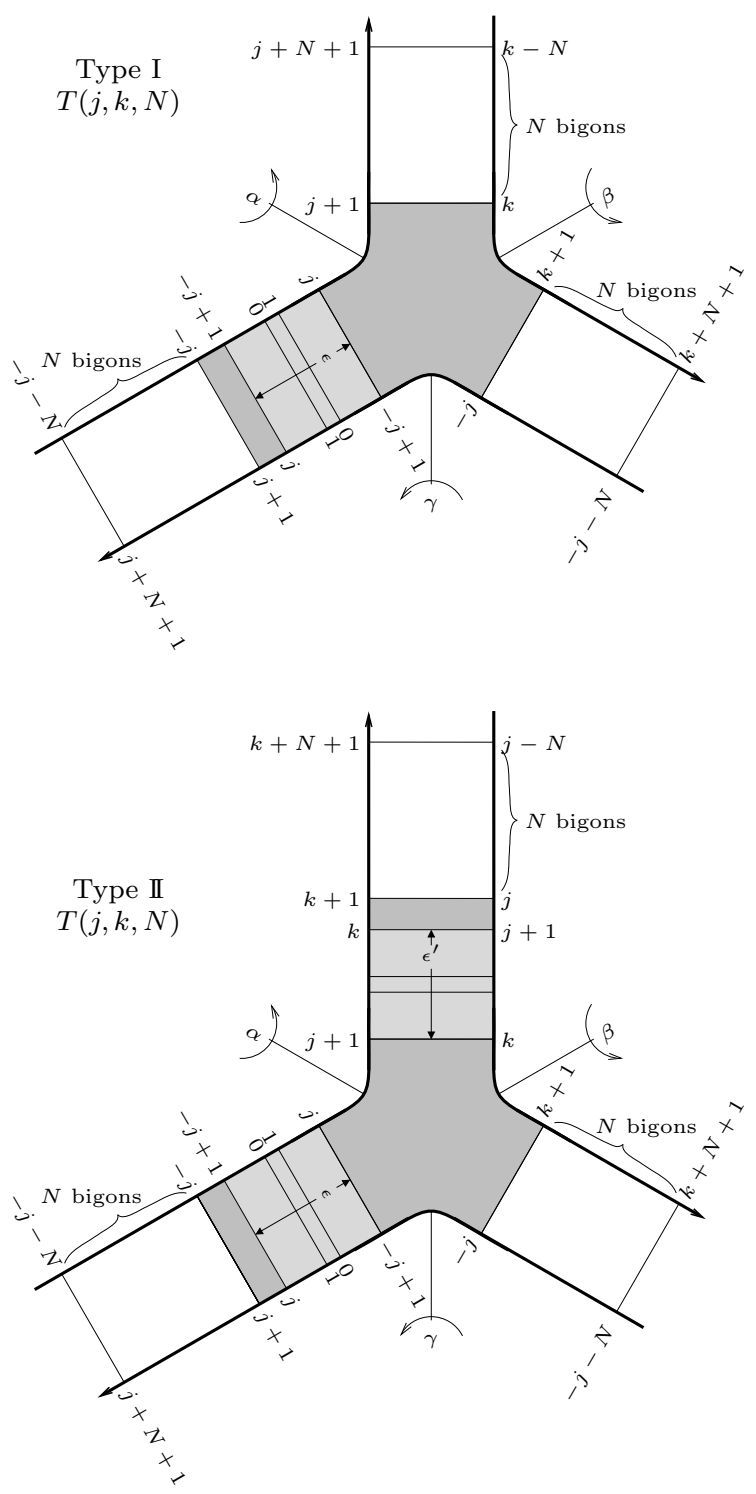

FiguRE 16

Definition 6.3. Let $T=T(j, k, N)$ be one of the two subgraphs of $\Lambda$ pictured in Figure [16, where $j>0, N \geq 0$ and either

(1) $0 \leq k<j$ and $T$ is the subgraph on the top. In this case we say that $T$ is of Type I. Furthermore, we require that $2 j+2 N+2 \leq t$, which is equivalent to no label appearing twice on any corner of $T$. Define $\epsilon$ to be the extended Scharlemann cycle in $T$ with corner $\overline{j,-j+1}$ in the $\alpha \gamma$-arm. Note that $T$ has $N+1$ bigons in the $\alpha \gamma$-arm beyond the extended Scharlemann cycle $\epsilon$, and $N$ bigons in the $\alpha \beta$ - and $\beta \gamma$-arms.

(2) $j<k \leq 3 j$ and $T$ is the subgraph on the bottom. In this case we say that $T$ is of Type II. Furthermore, we require that $j+k+2 N+2 \leq t$, which 

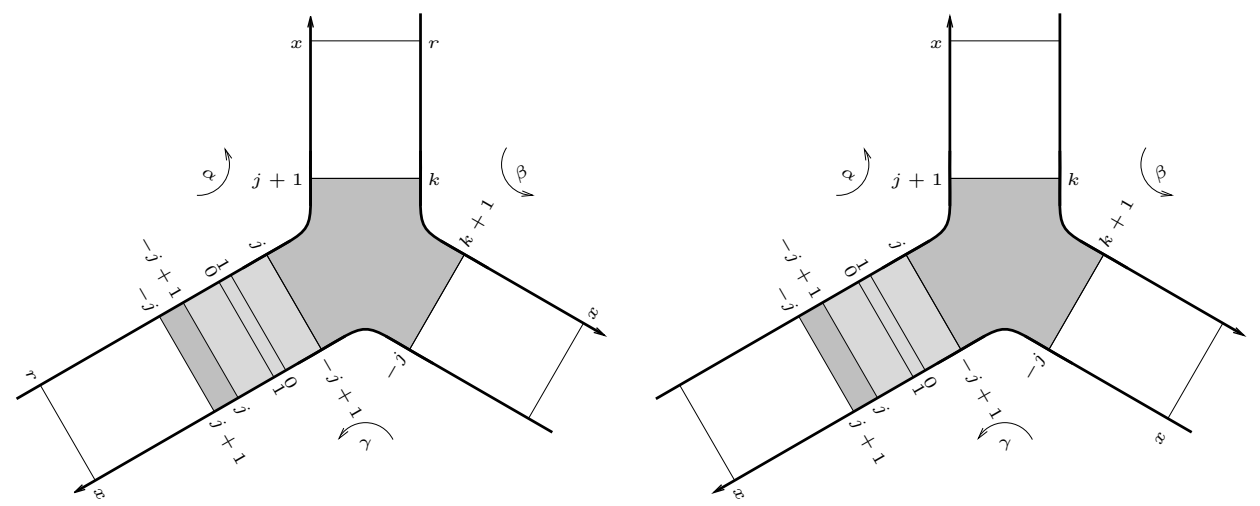

FiguRE 17

is equivalent to no label appearing twice on any corner of $T$. Define $\epsilon$ and $\epsilon^{\prime}$ to be the extended Scharlemann cycles in $T$ with corners $\overline{j,-j+1}$ and $\overline{j+1, k}$ in the $\alpha \gamma$ - and $\alpha \beta$-arms respectively. Note that $T$ has $N+1$ bigons in the $\alpha \gamma$ - and $\alpha \beta$-arms beyond the extended Scharlemann cycles $\epsilon$ and $\epsilon^{\prime}$, and $N$ bigons in the $\beta \gamma$-arm.

In this figure, $\alpha, \beta, \gamma$ are corners of vertices of $\Lambda$, and we refer to the $\alpha$-corner of $T$ as its ideal corner, denoted $c(T)$. Note that every corner of $T$ (as a labelled interval) is contained in $c(T)$. Note also that there are $2 N+2$ labels appearing in $T$ which are not labels in an extended Scharlemann cycle in $T$.

Lemma 6.4. Let $\sigma$ be a trigon face of $\Lambda_{x}$ that corresponds to a trigon of Type I or II. Let $n$ be the shortest distance (along a corner of $\sigma$ ) from $x$ to an extended Scharlemann cycle within $\sigma$. After possibly relabelling $\sigma$, there is a subgraph $T(j, k, N)$ of $\sigma$ such that $T(j, k, N)$ is either Type I or Type II according to whether $\sigma$ is Type I or Type II, and such that $N=n-1$. In particular, $x$ corresponds to a label in $c(T)$ (after relabelling).

Proof. Assume $\sigma$ is of Type I. Then we may assume after possibly relabelling that $\sigma$ is as in the left or right of Figure 17] according to whether $\sigma$ is an $x$-cycle or not (by Lemma 6.1). Note that $-j+1<k<j$ as $\sigma$ is of Type I. (If $k= \pm j$, then $\sigma$ would be of Type III. By parity, $k \neq j+1$ or $-j+1$. If $k \in \overline{(j+1) x}$, then there would be a second Scharlemann cycle on the $\alpha \beta$-arm. If $k \in \overline{x(-j-1)}$, then, regardless of whether $\sigma$ is an $x$-cycle or not, there is a second Scharlemann cycle along the $\beta \gamma$-arm.) Letting $N=x-(j+1), \sigma$ contains at least $N$ bigons along the $\alpha \beta$ - and $\beta \gamma$-arms, and $N+1$ out from the extended Scharlemann cycle on the $\alpha \gamma$-arm $(x-(k+1)>x-(j+1)=N$; then from the $\alpha \gamma$-arm deduce that the length of the interval from $x$ to $-j$ is at least $N+1$, the length of $\overline{j x})$. Furthermore, $N=n-1$. If $k<0$, then relabel using $i \mapsto 1-i$ and take the involution of the trigon interchanging the $\alpha$ and $\gamma$ corners. We then have the desired $T(j, k, N)$.

So assume $\sigma$ is of Type II. Then after possibly relabelling $\sigma$, we can write $\sigma$ as in Figure 18 where the width of the upper extended Scharlemann cycle is at most that of the lower and where either $r=r^{\prime}=x$ or $s=s^{\prime}=x$ (using Lemma 6.1 if $\sigma$ is not an $x$-edge cycle). The first condition gives that $k-(j+1) \leq j-(-j+1)$, implying $k \leq 3 j$ as desired. Let $N=\min \{-j-s, r-(k+1)\}$. Then there are 


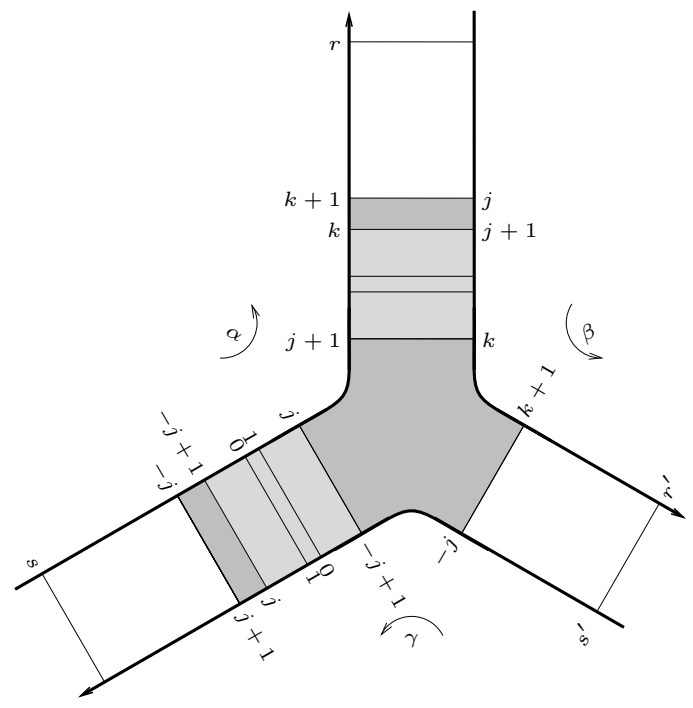

FIGURE 18

$N+1$ bigons beyond the extended Scharlemann cycle on each of the $\alpha \beta, \alpha \gamma-$ arms of $\sigma$. As either $r=r^{\prime}$ or $s=s^{\prime}$, there are $N$ bigons on the $\beta \gamma$-arm as well. Thus $\sigma$ contains $T(j, k, N)$ as desired. Finally, note that $N=n-1$.

Note that, whether $\sigma$ is Type I or II, the only label that appears twice on $\sigma$ is the $x$-label. Thus the only label that can appear twice in the constructed $T(j, k, N)$ must be the extremal ones on the ideal corner of $T$. As $j, k$ have the same parity, this is not possible.

Remark 6.5. In $T(j, k, N), k, j$ have the same parity by the Parity Rule of section 2.2 Thus $|j-k| \geq 2$.

Definition 6.6. Let $\mathcal{L}_{\mathrm{II}}=\left\{x \mid \Lambda_{x}\right.$ has a trigon face which is of Type I or Type II, but $x \notin L(\Sigma)\}$. Note that $x \notin L(\Sigma)$ means that $x$ is not a label in an extended Scharlemann cycle of length 2 or 3 .

Definition 6.7. A subgraph $\sigma$ of $\Lambda$ is said to be of Type $T(j, k, N)$ for some $j, k \in \mathbb{N}, N \in \mathbb{N} \cup\{0\}$ if after relabelling it becomes $T(j, k, N)$. For such a $\sigma$, let $c(\sigma)$ be the corner of $\sigma$ (before relabelling) corresponding to the ideal corner of $T(j, k, N)$. Let $\mathfrak{T}=\{\sigma \mid \sigma$ is of Type $T(j, k, N), j, k, N \in \mathbb{N}\}$. Let $\Sigma_{\mathbb{I I}}$ be a smallest subcollection of $\mathfrak{T}$ such that for any $x \in \mathcal{L}_{\mathbb{I I}}$ there is a $\sigma_{x} \in \Sigma_{\mathrm{II}}$ with $x \in c\left(\sigma_{x}\right)$. Lemma 6.4 guarantees the existence of $\Sigma_{\mathbb{I}}$. For $\sigma \in \Sigma_{\mathbb{I}}$, we denote by $j_{\sigma}, k_{\sigma}, N_{\sigma}$, its corresponding parameters. Thus $\left|\mathcal{L}_{\mathrm{II}}\right| \leq \sum_{\sigma \in \Sigma_{\mathrm{II}}}\left(2 N_{\sigma}+2\right)$.

Remark 6.8. Note that if three label intervals share a common label, then one of these intervals must be contained in the union of the other two. Thus the minimality of $\Sigma_{\mathbb{I}}$ guarantees that if $x \in \mathcal{L}_{\mathbb{I}}$, then $x \in c(\sigma)$ for at most 2 elements $\sigma$ of $\Sigma_{\mathbb{I}}$. Remember that $c(\sigma)$ contains all labels appearing on the corners of $\sigma$.

Definition 6.9. Partition $\Sigma_{\mathrm{II}}$ as follows:

$\Sigma_{\mathbb{I}}(\mathrm{I})=\left\{\sigma \in \Sigma_{\mathbb{I}} \mid \sigma\right.$ is of Type I $\}$ and $\Sigma_{\mathbb{I}}(\mathbb{I})=\left\{\sigma \in \Sigma_{\mathbb{I}} \mid \sigma\right.$ is of Type $\left.\mathbb{I}\right\}$

$\Sigma_{\mathbb{I}}(\mathrm{I}, 1)=\left\{\sigma \in \Sigma_{\mathbb{I}}(\mathrm{I}) \mid N_{\sigma}<j_{\sigma}-k_{\sigma}\right\}$ 


$$
\begin{aligned}
& \Sigma_{\mathbb{I}}(\mathrm{I}, 2)=\left\{\sigma \in \Sigma_{\mathbb{I}}(\mathrm{I}) \mid 2 k_{\sigma}-j_{\sigma} \leq 0 ; j_{\sigma}-k_{\sigma} \leq N_{\sigma} \leq 4 j_{\sigma}-2 k_{\sigma}-1\right\} \\
& \Sigma_{\mathbb{I}}(\mathrm{I}, 3)=\left\{\sigma \in \Sigma_{\mathbb{I}}(\mathrm{I}) \mid 2 k_{\sigma}-j_{\sigma}>0 ; j_{\sigma}-k_{\sigma} \leq N_{\sigma}<k_{\sigma}\right\} \\
& \Sigma_{\mathbb{I}}(\mathrm{I}, 4)=\left\{\sigma \in \Sigma_{\mathbb{I}}(\mathrm{I}) \mid 2 k_{\sigma}-j_{\sigma}>0 ; k_{\sigma} \leq N_{\sigma} \leq 4 j_{\sigma}-2 k_{\sigma}-1\right\} \\
& \Sigma_{\mathbb{I}}(\mathrm{I}, 5)=\left\{\sigma \in \Sigma_{\mathbb{I}}(\mathrm{I}) \mid 2 k_{\sigma}-j_{\sigma}>0 ; 4 j_{\sigma}-2 k_{\sigma}-1<N_{\sigma}\right\} \\
& \Sigma_{\mathbb{I}}(\mathrm{I}, 6)=\left\{\sigma \in \Sigma_{\mathbb{I}}(\mathrm{I}) \mid 2 k_{\sigma}-j_{\sigma} \leq 0 ; 4 j_{\sigma}-2 k_{\sigma}-1<N_{\sigma}\right\} \\
& \Sigma_{\mathbb{I}}(\mathbb{I I}, 1)=\left\{\sigma \in \Sigma_{\mathbb{I}}(\mathrm{II}) \mid N_{\sigma}<k_{\sigma}-j_{\sigma}\right\} \\
& \Sigma_{\mathbb{I}}(\mathrm{I}, 2)=\left\{\sigma \in \Sigma_{\mathbb{I}}(\mathbb{I}) \mid 2 j_{\sigma}-k_{\sigma} \leq 0 ; k_{\sigma}-j_{\sigma} \leq N_{\sigma} \leq 3 k_{\sigma}-j_{\sigma}-1\right\} \\
& \Sigma_{\mathbb{I}}(\mathbb{I}, 3)=\left\{\sigma \in \Sigma_{\mathbb{I}}(\mathrm{II}) \mid 2 j_{\sigma}-k_{\sigma}>0 ; k_{\sigma}-j_{\sigma} \leq N_{\sigma}<k_{\sigma}\right\} \\
& \Sigma_{\mathbb{I}}(\mathbb{I I}, 4)=\left\{\sigma \in \Sigma_{\mathbb{I}}(\mathrm{II}) \mid 2 j_{\sigma}-k_{\sigma}>0 ; k_{\sigma} \leq N_{\sigma} \leq 3 k_{\sigma}-j_{\sigma}-1\right\} \\
& \Sigma_{\mathbb{I}}(\mathbb{I}, 5)=\left\{\sigma \in \Sigma_{\mathbb{I}}(\mathbb{I}) \mid 2 j_{\sigma}-k_{\sigma}>0 ; 3 k_{\sigma}-j_{\sigma}-1<N_{\sigma}\right\} \\
& \Sigma_{\mathbb{I}}(\mathbb{I}, 6)=\left\{\sigma \in \Sigma_{\mathbb{I}}(\mathrm{II}) \mid 2 j_{\sigma}-k_{\sigma} \leq 0 ; 3 k_{\sigma}-j_{\sigma}-1<N_{\sigma}\right\} .
\end{aligned}
$$

We will abbreviate $\Sigma_{\mathrm{II}}(i, j)$ as $(i, j)$.

One checks that Definition 6.9 does indeed partition $\Sigma_{\mathrm{II}}$. That is,

Lemma 6.10. $\Sigma_{\mathbb{I}}(i, j) \cap \Sigma_{\mathbb{I}}(r, s) \neq \emptyset$ iff $i=r, j=s$. Furthermore, $\Sigma_{\mathbb{I}}=\bigcup \Sigma_{\mathbb{I}}(i, j)$.

To each of the partition elements of Definition 6.9 we associate collections of essential closed curves that will eventually allow us to bound $\sum_{\Sigma_{\mathrm{II}}} N_{\sigma}$ in Theorem 6.24 at the end of this section.

Claim 6.11. Let $T(j, k, N)$ corresponding to $\sigma \in \Sigma_{\text {II }}$ be of Type I. Let $\epsilon$ be the maximal extended Scharlemann cycle for $\sigma$. For each $0 \leq s \leq \min \{N, j-k-1\}$ there is a configuration $H(s)$ in $\widehat{F}$ consisting of embedded curves $C(s), C^{\prime}(s)$ in $a(\epsilon)$ connected by a path of three edges of $\sigma$. When $k \neq 0, C(s)$ and $C^{\prime}(s)$ are disjoint, and when $k=0$, they are identical. Furthermore:

(1) If $H\left(s_{1}\right), H\left(s_{2}\right), s_{2}>s_{1}$, share any vertices or edges then either

- $s_{2}=s_{1}+2 k$, and $C\left(s_{1}\right)=C^{\prime}\left(s_{2}\right)$ with no other shared vertices or edges; or

- $s_{1}+s_{2}=2 k-1$, and $C^{\prime}\left(s_{1}\right)=C^{\prime}\left(s_{2}\right)$ with no other shared vertices or edges.

In particular,

$$
\mathcal{C}_{\sigma}=\{C(s) \mid 0 \leq s \leq \min \{N, j-k-1\}\}
$$

is a collection of $\min \{N+1, j-k\}$ disjoint curves. Thus

$$
\left|\mathcal{C}_{\sigma}\right|= \begin{cases}N+1 & \text { if } \sigma \in(\mathrm{I}, 1), \\ j-k & \text { if } \sigma \in(\mathrm{I})-(\mathrm{I}, 1) .\end{cases}
$$

(2) If $H(s)$ shares a vertex with an element of $a(\epsilon)$ then that element is either $C(s)$ or $C^{\prime}(s)$.

(3) No $H(s)$ lies in an annulus on $\widehat{F}$.

Proof. It is convenient to work with $\sigma$ after it is relabelled as $T(j, k, N)$; however, $H(s)$ is taken to be under the original labelling.

For $0 \leq s \leq \min \{N, j-k-1\}$, let $C(s)$ be the essential curve of $a(\epsilon)$ formed by the two $(k+1+s,-k-s)$-edges in $\epsilon$ and let $C^{\prime}(s)$ be the essential curve of $a(\epsilon)$ formed by the two $(k-s,-k+1+s)$-edges. $C(s)$ and $C^{\prime}(s)$ share no edges or vertices as long as $k \neq 0$. Otherwise, $C(s)$ and $C^{\prime}(s)$ are the same. The distinct edges $e_{1}, e_{2}, e_{3}$ of Figure 19 now join $C(s)$ and $C^{\prime}(s)$ on $\widehat{F}$. Define $H(s)$ to be the configuration on $\widehat{F}$ given by $C(s), C^{\prime}(s), e_{1}, e_{2}, e_{3}$ along with the vertices to which they are incident as shown in Figure 20. When $k=0, C(s)$ and $C^{\prime}(s)$ are the same 


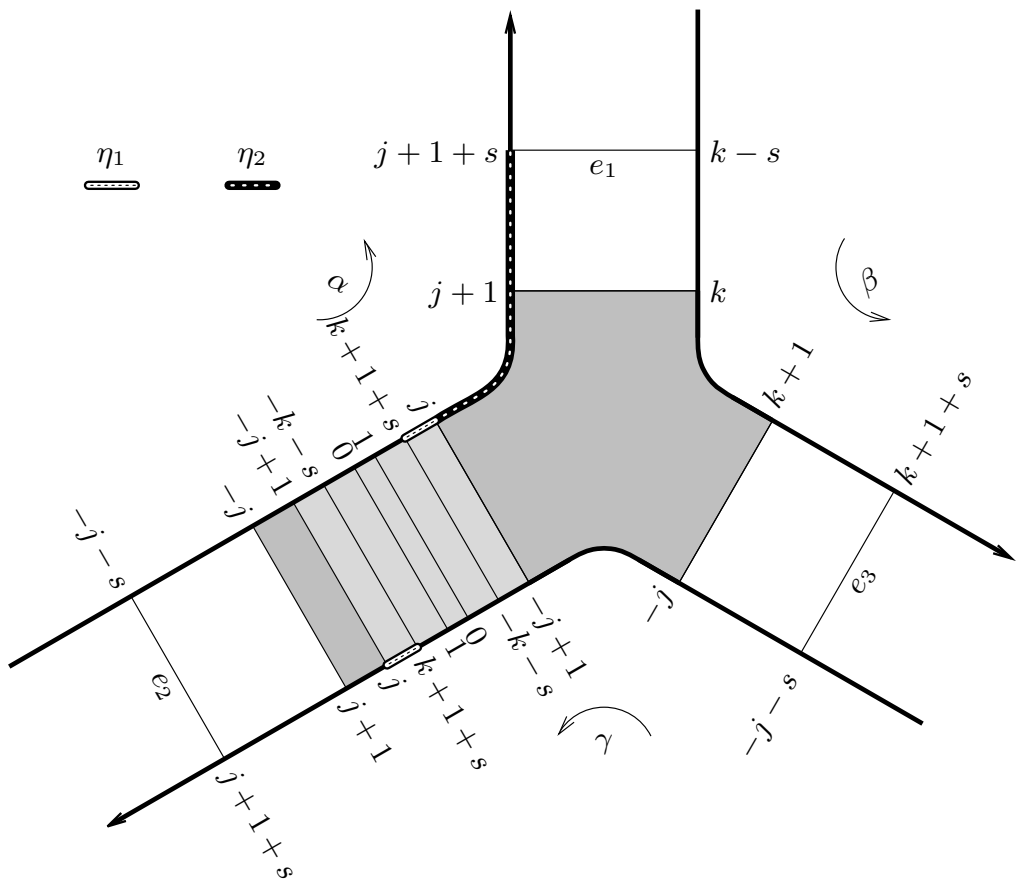

FigURE 19

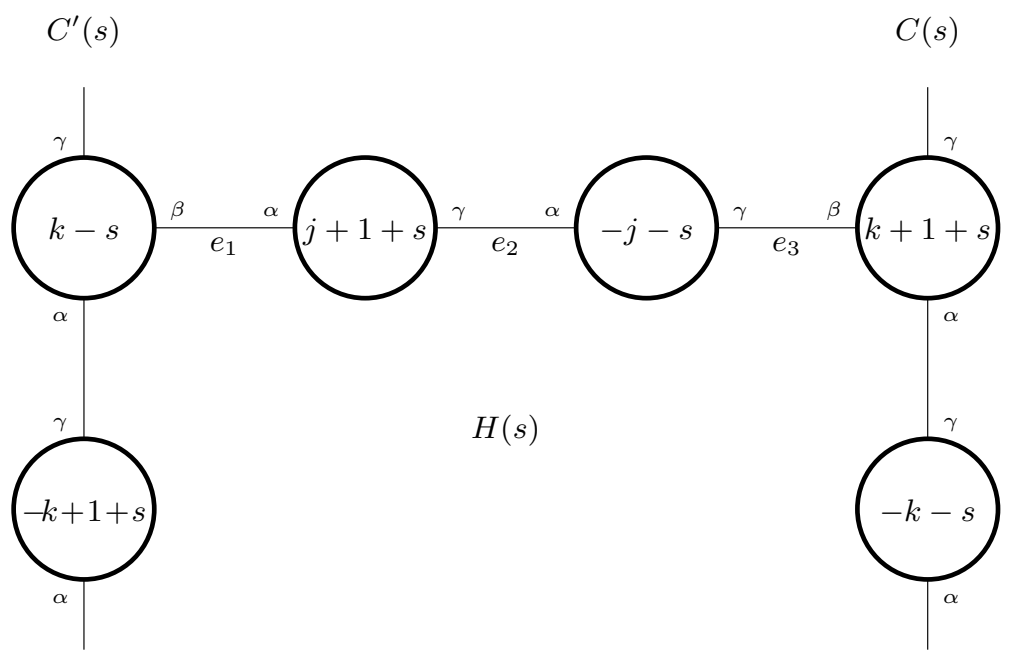

FiguRE 20

and the edges $e_{1}$ and $e_{3}$ are incident to different vertices of $C(s)$ and on opposite sides of $C(s)$. Thus when $k=0, H(s)$ cannot lie in an annulus on $\widehat{F}$.

One checks that $H\left(s_{1}\right), H\left(s_{2}\right)$ share vertices or edges only as described in item (1) above. In particular, $C\left(s_{1}\right), C\left(s_{2}\right)$ are disjoint. To check (2), note that for $H(s)$ the labels of $e_{i}$ are either those of $C(s)$ or $C^{\prime}(s)$ or lie outside the labels of $\epsilon$. 


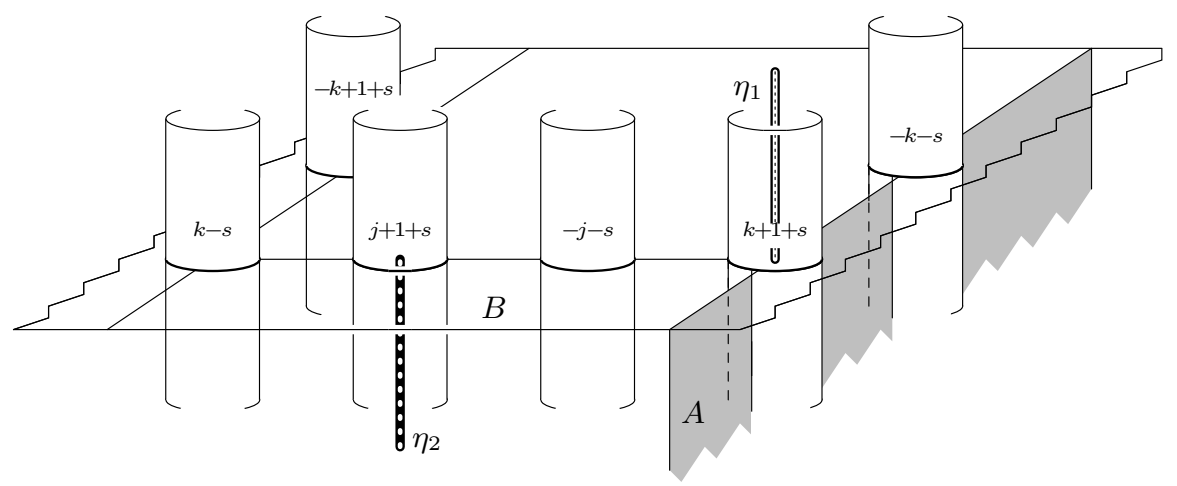

FiguRE 21

Now fix $s$ and assume that $H(s)$ lies in an annulus on $\widehat{F}$. As noted above, $k \neq 0$. Then $C^{\prime}(s)$ and $C(s)$ are different and cobound an annulus $B$ on $\widehat{F}$. See Figure 20,

As the edges $e_{1}, e_{2}, e_{3}$ lie in $B$, no vertex from $-j+1$ to $j$ can lie in the interior of $B$. Let $A$ be the annulus contained in $A(\epsilon)$ with boundary $C^{\prime}(s) \cup C(s)$ obtained from the union of the appropriate bigons in $\epsilon$. Note the annuli $A$ and $B$ will have disjoint interiors (by (2)).

The orientations of $C^{\prime}(s)$ and $C(s)$ given by moving along an edge from labels $\alpha$ to $\gamma$ must be in the same direction along $B$ since otherwise $A \cup B$ would form a Klein bottle. This forces the labeling of the edges in Figure 20 to be as pictured. (This labeling is also forced because $k-s$ and $k+1+s$ have opposite parity.)

Let $\mathcal{T}$ be the torus $A \cup B$ which is necessarily separating. We will arrive at a contradiction by finding an embedded curve that intersects $\mathcal{T}$ once.

Let $\eta_{1}$ be the arc which is the corner of Figure 19 along $\alpha$ that runs between the labels $k+1+s$ and $j$ within the extended Scharlemann cycle $\epsilon$. Noting that the curves $C(s+1), \ldots, C(j-k-1)$ must lie outside of $B$ (since they are essential on $\widehat{F}$ and disjoint from $\left.e_{1}, e_{2}, e_{3}\right)$, we see that $\eta_{1}$ lies on one side of $\mathcal{T}$. In particular, $\eta_{1}$ intersects $\mathcal{T}$ only at the vertex $k+1+s$.

Let $\eta_{2}$ be the arc that is the corner in Figure 19 along $\alpha$ running between labels $j+1+s$ and $j$. When $s<k$ the vertices $k+1-s, \ldots, k$ must lie outside of $B$, hence so do the edges of $T=T(j, k, N)$ which are incident to $\eta_{2}$ in Figure 19. Therefore $\eta_{2}$ lies entirely on one side of $\mathcal{T}$. In particular, $\eta_{2}$ intersects $\mathcal{T}$ only at the vertex $j+1+s$. When $s \geq k$, then the vertices $k+1-s, \ldots, k$ may share vertex $-k+1+s$ with $B$ (this happens when $2 k-1 \geq s \geq k$ ). But the edge in $T$ connecting $-k+1+s$ to $2 k+j-s$ in $\eta_{2}$ must lie outside of $B$ on $G_{F}$ as it is incident to vertex $-k+1+s$ at label $\beta$ - which from the labelling in Figure 20 directs this edge outside of $B$. Again, we conclude that $\eta_{2}$ intersects $\mathcal{T}$ only at vertex $j+1+s$.

The $\operatorname{arcs} \eta_{1}$ and $\eta_{2}$ approach their endpoints at the vertices $k+1+s$ and $j+1+s$, respectively, in opposite directions along $K$. Since the vertices $k+1+s$ and $j+1+s$ have the same parity and lie on $B \subset \widehat{F}, \eta_{1}$ and $\eta_{2}$ lie on opposite sides of $\mathcal{T}$. Hence $\eta_{1} \cup \eta_{2} \cup e_{2} \cup e_{3}$ forms a loop that may be perturbed to transversely intersect $\mathcal{T}$ just once. See Figure 21.

Claim 6.12. Let $T(j, k, N)$ corresponding to $\sigma \in \Sigma_{\mathrm{II}}$ be of Type II. Let $\epsilon, \epsilon^{\prime}$ be the extended Scharlemann cycles for $\sigma$ with corners $\overline{-j+1, j}, \bar{j}+1, k$ (resp.) in 


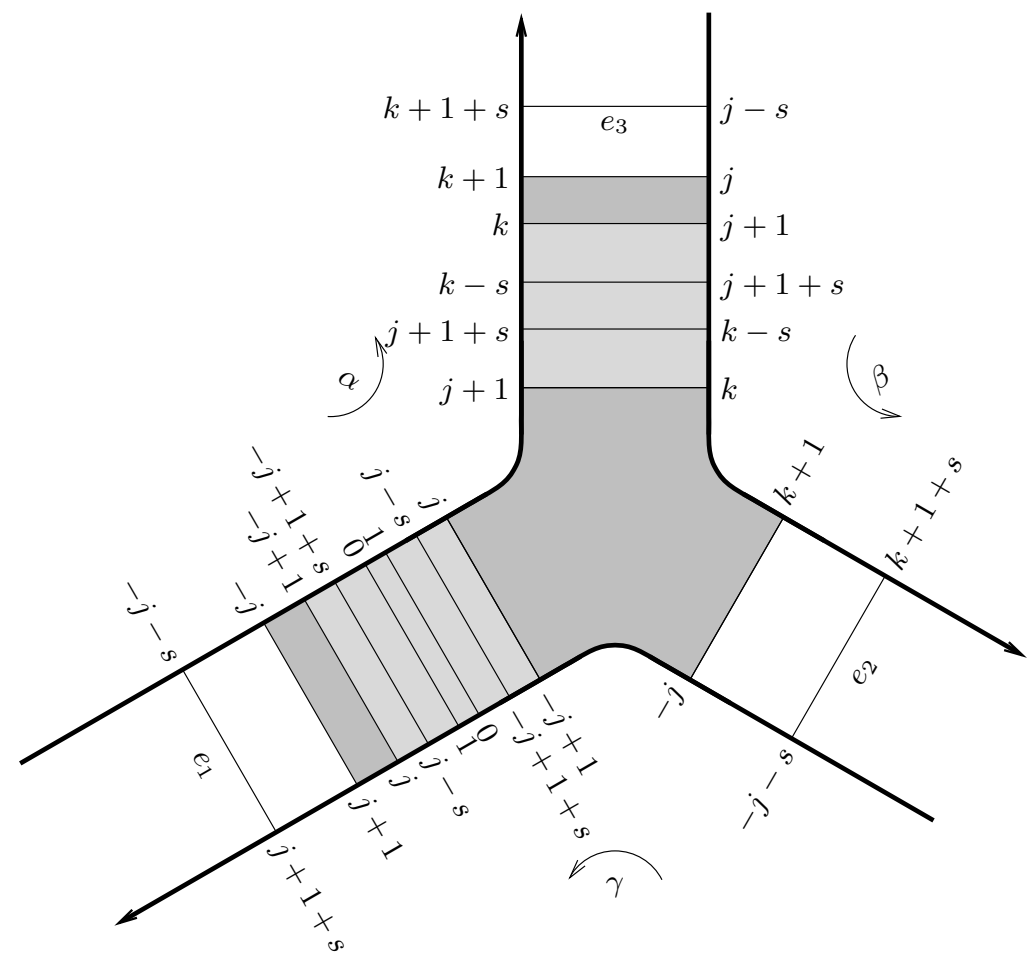

FIGURE 22

$T(j, k, N)$. For each $0 \leq s \leq \min \left\{N, \frac{1}{2}(k-j)-1\right\}$ there is a configuration $H(s)$ in $\widehat{F}$ consisting of disjoint curves $C(s), C^{\prime}(s)$ in $a(\epsilon), a\left(\epsilon^{\prime}\right)$ (resp.) connected by a path of three edges of $\sigma$. Furthermore:

(1) $H\left(s_{1}\right), H\left(s_{2}\right)$ share no vertices or edges when $s_{1} \neq s_{2}$. In particular,

$$
\mathcal{C}_{\sigma}=\left\{C(s), C^{\prime}(s) \mid 0 \leq s \leq \min \left\{N, \frac{1}{2}(k-j)-1\right\}\right\}
$$

is a collection of $\min \{2 N+2, k-j\} \geq \min \{N+1, k-j\}$ disjoint curves on $\widehat{F}$. Thus

$$
\left|\mathcal{C}_{\sigma}\right| \geq \begin{cases}N+1 & \text { if } \sigma \in(\mathrm{II}, 1), \\ k-j & \text { if } \sigma \in(\mathrm{II})-(\mathrm{II}, 1) .\end{cases}
$$

(2) If $H(s)$ shares a vertex with an element of $a(\epsilon)$ or $a\left(\epsilon^{\prime}\right)$, then that element is either $C(s)$ or $C^{\prime}(s)$.

(3) No $H(s)$ lies in an annulus on $\widehat{F}$.

Proof. We work with $\sigma$ after it is relabelled as $T(j, k, N)$; however, $H(s)$ is taken to be under the original labelling.

For $0 \leq s \leq \min \left\{N, \frac{1}{2}(k-j)-1\right\}$, let $C(s)$ be the essential curve of $a(\epsilon)$ formed by the two $(j-s,-j+1+s)$-edges in $\epsilon$, and let $C^{\prime}(s)$ be the essential curve of $a\left(\epsilon^{\prime}\right)$ formed by the two $(j+1+s, k-s)$-edges in $\epsilon^{\prime}$. 


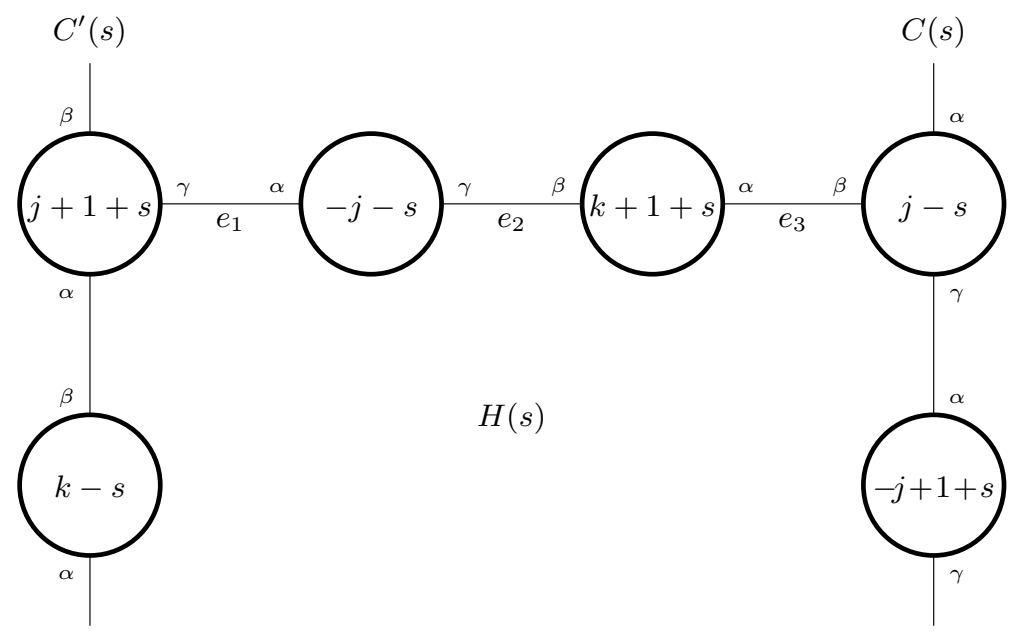

FIGURE 23

Define $H(s)$ to be the connected subgraph of $\widehat{F}$ which is the union of $C(s), C^{\prime}(s)$, the edges $e_{1}, e_{2}, e_{3}$ of Figure 22 and the vertices they connect as shown in Figure 23 . Note that each of these edges is in $T(j, k, N)$ since $s \leq N$ and that they are all distinct. One now checks that $H\left(s_{1}\right), H\left(s_{2}\right)$ share no vertices or edges if $s_{1} \neq s_{2}$. Also the two vertices of $H(s)$ not in $C(s) \cup C^{\prime}(s)$ lie outside $a(\epsilon)$ and $a\left(\epsilon^{\prime}\right)$ as they are labeled $-j-s$ and $k+1+s$, which implies conclusion (2).

Assume some $H(s)$ lies in an annulus on $\widehat{F}$. Then $C(s), C^{\prime}(s)$ are parallel on $\widehat{F}$, and Corollary 5.15]implies that $\epsilon$ and $\epsilon^{\prime}$ have the same core labels, a contradiction.

Lemma 6.13.

$$
\sum_{\sigma \in \Sigma_{\mathbb{I I}}} \min \left\{N_{\sigma}+1,\left|j_{\sigma}-k_{\sigma}\right|\right\} \leq \sum_{\sigma \in \Sigma_{\mathrm{II}}}\left|\mathcal{C}_{\sigma}\right| \leq 36(g-1) .
$$

Proof. Let $\mathcal{C}=\bigcup_{\sigma \in \Sigma_{\pi}} \mathcal{C}_{\sigma}$, with $\mathcal{C}_{\sigma}$ as in Claims 6.11, 6.12, By Claim 6.11, Claim 6.12 and Remark 6.8 any vertex belongs to at most two elements of $\mathcal{C}$.

Partition $\mathcal{C}$ as $\mathcal{C}_{0} \cup \mathcal{C}_{1} \cup \mathcal{C}_{2}$, where $\mathcal{C}_{0}=\{C \in \mathcal{C}: C$ is disjoint from all other elements of $\mathcal{C}\}, \mathcal{C}_{1}=\left\{C \in \mathcal{C}\right.$ : there exists $C^{\prime} \in \mathcal{C}$ such that $C$ and $C^{\prime}$ meet at a single vertex $\}$, and $\mathcal{C}_{2}=\left\{C \in \mathcal{C}\right.$ : there exists $C^{\prime} \in \mathcal{C}, C^{\prime} \neq C$, such that $C$ and $C^{\prime}$ meet at two vertices $\}$. Note that for $i \neq j$, the elements of $\mathcal{C}_{i}$ are disjoint from the elements of $\mathcal{C}_{j}$.

Let $\mathcal{D}_{2}$ be a subcollection of disjoint curves in $\mathcal{C}_{2}$ with $\left|\mathcal{D}_{2}\right|=\left|\mathcal{C}_{2}\right| / 2$.

Let $\mathcal{D}_{1}$ be the collection of simple closed curves on $\widehat{F}$ obtained from the elements of $\mathcal{C}_{1}$ by removing all non-transverse points of intersection by small perturbations. Thus any two elements of $\mathcal{D}_{1}$ either intersect transversely at a single point or are disjoint. We may abstractly represent $\mathcal{D}_{1}$ by a graph $\Gamma$ whose nodes correspond to the elements of $\mathcal{D}_{1}$ and where two nodes are joined by an edge if and only if the corresponding elements of $\mathcal{D}_{1}$ intersect. Partition $\mathcal{D}_{1}$ as $\mathcal{D}_{1}^{(0)} \cup \mathcal{D}_{1}^{(1)}$, where $\mathcal{D}_{1}^{(0)}$ consists of the elements of $\mathcal{D}_{1}$ that correspond to isolated nodes of $\Gamma$. The elements 
of $\mathcal{D}_{1}^{(1)}$ then correspond to components of $\Gamma$ that are either paths with $k$ nodes, $k \geq 2$, or cycles with $k$ nodes, $k \geq 3$. In each path (resp. cycle) component of $\Gamma$ with $k$ nodes, we can choose $\left\lfloor\frac{k+1}{2}\right\rfloor$ (resp. $\left\lfloor\frac{k}{2}\right\rfloor$ ) nodes such that the corresponding elements of $\mathcal{D}_{1}^{(1)}$ are disjoint. Let the resulting subcollection of $\mathcal{D}_{1}^{(1)}$ be $\mathcal{E}_{1}$.

Let $\mathcal{F}=\mathcal{C}_{0} \cup \mathcal{D}_{1}^{(0)} \cup \mathcal{D}_{2}$. Note that since any element of $\mathcal{E}_{1}$ has algebraic intersection number 1 with some element of $\mathcal{D}_{1}^{(1)}$, no element of $\mathcal{E}_{1}$ is parallel on $\widehat{F}$ to any element of $\mathcal{F}$. Furthermore, if two elements of $\mathcal{E}_{1}$ are parallel on $\widehat{F}$, then the corresponding nodes of $\Gamma$ belong to either a path component of $\Gamma$ with three nodes or a cycle component with four nodes. Removing one element from each parallel pair gives a subcollection $\mathcal{E}$ of $\mathcal{E}_{1}$ with $|\mathcal{E}| \geq\left|\mathcal{D}_{1}^{(1)}\right| / 4$, such that no element of $\mathcal{E}$ is parallel to either another element of $\mathcal{E}$ or an element of $\mathcal{F}$.

Let $C \in \mathcal{D}_{1}^{(0)}$. Then there exists $C^{\prime} \in \mathcal{C}_{1}$ such that $C$ and $C^{\prime}$ intersect, nontransversely, at a single vertex. If $C \cup C^{\prime}$ were contained in an annulus in $\widehat{F}$, then by Corollary 5.15 the corresponding core Scharlemann cycles would have to have the same label pair and hence $C$ and $C^{\prime}$ would share two vertices, a contradiction. It follows that in any parallelism class of elements of $\mathcal{F}$ the corresponding elements of $\mathcal{C}$ (i.e., before perturbing to remove non-transverse intersections) are disjoint.

We claim that no more than six elements of $\mathcal{F}$ can be parallel on $\widehat{F}$. For suppose we have seven elements of $\mathcal{F}$ that are parallel. Let $C_{r}, C_{l}$ be the outermost elements of this group. Let $C_{1}, \ldots, C_{5}$ be elements of $\mathcal{C}$ between these. Let $C_{i} \in \mathcal{C}_{\sigma_{i}} \subset \mathcal{C}$ and $C_{i} \subset H\left(s_{i}\right)$. In the notation of Claims 6.11, 6.12 if $\sigma_{i}$ is of Type I, then $C_{i}=C\left(s_{i}\right)$; if $\sigma_{i}$ is of Type II, then $C_{i}$ is $C\left(s_{i}\right)$ or $C^{\prime}\left(s_{i}\right)$. Then for each $i, H\left(s_{i}\right)$ must share a vertex with either $C_{r}$ or $C_{l}$; otherwise $H\left(s_{i}\right)$ lies in an annulus, contradicting Claims 6.11 6.12. As $C_{i}$ is disjoint from $C_{r}, C_{l}$, we can assign to each $i=1, \ldots, 5$ a vertex $v_{i} \in H\left(s_{i}\right)$ which lies in $C_{r}$ or $C_{l}$ and is the closest such to $C_{i}$ in the path metric of $H\left(s_{i}\right)$. As $C_{r}$ and $C_{l}$ each contain only two vertices, it must be that, without loss of generality, $v_{1}=v_{2} \in C_{r}$. Let $C_{r} \in \mathcal{C}_{\sigma_{r}}$ with $C_{r} \subset H\left(s_{r}\right)$. By Claims 6.11, 6.12, $\sigma_{r} \neq \sigma_{1}, \sigma_{2}$. (Say $\sigma_{1}=\sigma_{r}$. Then $v_{1} \in H\left(s_{1}\right) \cap H\left(s_{r}\right)$, implying that $\left\{C_{1}, C_{r}\right\}=\left\{C\left(s_{1}\right), C^{\prime}\left(s_{1}\right)\right\}$ are parallel. This and the definition of $v_{1}$ imply that $H\left(s_{1}\right)$ lies in an annulus.) Then Remark 6.8 implies that $\sigma_{1}=\sigma_{2}$. By Claims 6.11, 6.12, it must be that either $\sigma_{1}$ is of Type I and $v_{1}=v_{2} \in C^{\prime}\left(s_{1}\right)=C^{\prime}\left(s_{2}\right)$, or that $\sigma_{1}$ is of Type II and $H\left(s_{1}\right)=H\left(s_{2}\right)$. In either case, it must be that one of $H\left(s_{1}\right)$ or $H\left(s_{2}\right)$, say $H\left(s_{1}\right)$, intersects $C_{l}$ in a vertex closer to $C_{1}$ in $H\left(s_{1}\right)$ than $v_{1}$, a contradiction.

Hence there exists a subcollection $\mathcal{F}^{\prime}$ of $\mathcal{F}$ with $\left|\mathcal{F}^{\prime}\right| \geq|\mathcal{F}| / 6$ such that no two elements of $\mathcal{F}^{\prime}$ are parallel. Then $\mathcal{E} \cup \mathcal{F}^{\prime}$ is a collection of disjoint, essential, nonparallel simple closed curves on $\widehat{F}$. Hence $|\mathcal{E}|+\left|\mathcal{F}^{\prime}\right| \leq 3(g-1)$. Therefore

$$
\begin{aligned}
36(g-1) & \geq 12|\mathcal{E}|+12\left|\mathcal{F}^{\prime}\right| \\
& \geq 3\left|\mathcal{D}_{1}^{(1)}\right|+2|\mathcal{F}| \\
& =3\left|\mathcal{D}_{1}^{(1)}\right|+2\left(\left|\mathcal{C}_{0}\right|+\left|\mathcal{D}_{1}^{(0)}\right|+\left|\mathcal{D}_{2}\right|\right) \\
& \geq 2\left|\mathcal{C}_{0}\right|+2\left|\mathcal{C}_{1}\right|+\left|\mathcal{C}_{2}\right| \\
& \geq\left|\mathcal{C}_{0}\right|+\left|\mathcal{C}_{1}\right|+\left|\mathcal{C}_{2}\right|=|\mathcal{C}|
\end{aligned}
$$

as claimed. 
Claim 6.14. Let $T(j, k, N)$ corresponding to $\sigma \in \Sigma_{\mathrm{II}}$ be of Type I such that $N \geq j-k$ and $2 k-j>0$. Let $\epsilon$ be the maximal extended Scharlemann cycle for $\sigma$. For each $0 \leq s \leq \min \{N-(j-k), 2 k-j-1\}$ there is a configuration $H(s)$ in $\widehat{F}$ consisting of disjoint curves $C(s), C^{\prime}(s)$ in $a(\epsilon)$ connected by a path of four edges of $\sigma$.

(1) If $H\left(s_{1}\right), H\left(s_{2}\right), s_{2}>s_{1}$, share any vertices or edges, then $s_{2}=s_{1}+j-k$. In this case $C\left(s_{1}\right)=C^{\prime}\left(s_{2}\right)$ and the edge $e_{1}$ of $H\left(s_{1}\right)$ is the edge $e_{4}$ of $H\left(s_{2}\right)$. Besides these edges and the vertices to which they are incident, there are no other shared vertices or edges between $H\left(s_{1}\right), H\left(s_{2}\right)$. In particular,

$$
\mathcal{B}_{\sigma}=\{C(s) \mid 0 \leq s \leq \min \{N-(j-k), 2 k-j-1\}\}
$$

is a collection of $\min \{N-(j-k)+1,2 k-j\}$ disjoint curves. Thus

$$
\left|\mathcal{B}_{\sigma}\right|= \begin{cases}N-(j-k)+1 & \text { if } \sigma \in(\mathrm{I}, 3) \\ 2 k-j & \text { if } \sigma \in(\mathrm{I}, 4) \cup(\mathrm{I}, 5) .\end{cases}
$$

(2) Each vertex of $G_{F}$ lies in at most one element of $\mathcal{B}_{\sigma}$.

(3) If $H(s)$ shares a vertex with an element of $a(\epsilon)$, then that element is either $C(s)$ or $C\left(s^{\prime}\right)$.

(4) No $H(s)$ lies in an annulus on $\widehat{F}$.

Proof. We work with $\sigma$ after it is relabelled as $T(j, k, N)$; however, $H(s)$ is taken to be under the original labelling. Note that as $\sigma$ is Type I, $j>k$.

For $0 \leq s \leq \min \{N-(j-k), 2 k-j-1\}$ let $C(s)$ be the essential curve of $a(\epsilon)$ on $\widehat{F}$ formed by the two $(2 k-j-s,-2 k+j+1+s)$-edges in $\epsilon$ (note that $\max \{1, k-N\} \leq 2 k-j-s \leq 2 k-j=k-(j-k)<k)$. Let $C^{\prime}(s)$ be the essential curve of $a(\epsilon)$ formed by the two $(k-s,-k+s+1)$-edges in $\epsilon$ (note that $3 \leq \max \{j-k+1, j-N\} \leq k-s \leq k\}$ ). The four edges $e_{1}, e_{2}, e_{3}, e_{4}$ shown in Figure 24, along with $C(s), C^{\prime}(s)$, give the configuration, $H(s)$, in the graph $G_{F}$ depicted in Figure 25. The vertices these edges connect are included in $H(s)$.

Assume $H\left(s_{1}\right), H\left(s_{2}\right), s_{1}<s_{2}$, share vertices. This can only happen if $s_{2}=$ $s_{1}+j-k$ and the shared vertices and edges are as claimed. In particular, $C\left(s_{2}\right)$ shares no vertices (hence no edges) with $C\left(s_{1}\right)$. This verifies conclusions (1) and (2). As the edges $e_{1}, e_{2}, e_{3}, e_{4}$ lie outside of $\epsilon$, conclusion (3) holds.

Assume for contradiction that $H(s)$ lies in an annulus in $\widehat{F}$. Let $B$ be the annulus in $\widehat{F}$ cobounded by $C(s), C^{\prime}(s)$. Let $A$ be the annulus contained in $A(\epsilon)$ with boundary $C(s) \cup C^{\prime}(s)$ obtained from the union of the bigons in $\epsilon$ with labels $-j+2 k-s,-j+2 k+1-s, \ldots, k-s$ and $j-2 k+1+s, j-2 k+s, \ldots, 1-k+s$. The labeling of Figure 26] shows that the induced orientation on $\partial A$ does not cancel an orientation on $\partial B$. Note that in Figure 26] we use that $j-k$ is even, and hence that vertices $-j+2 k-s$ and $k-s$ are parallel. Thus $A \cup B$ is a Klein bottle. This is a contradiction. 


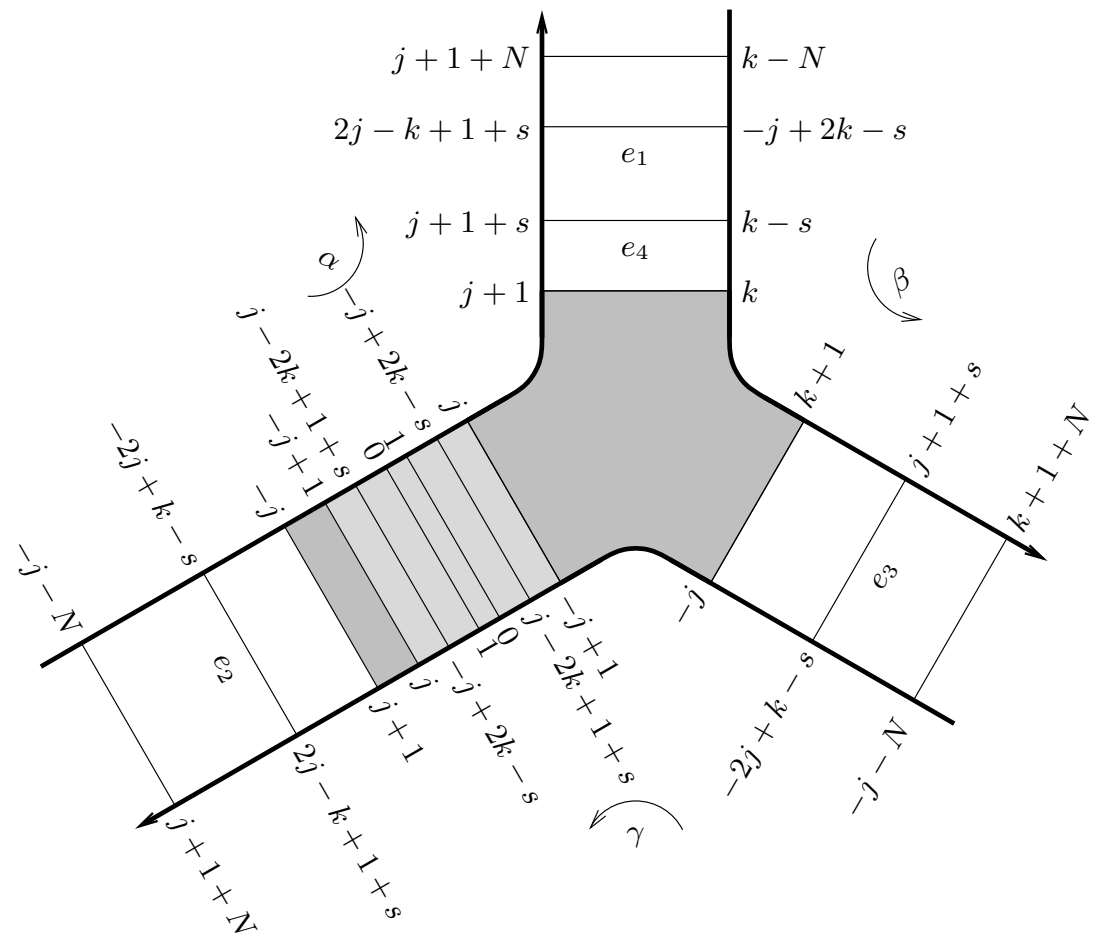

FIGURE 24

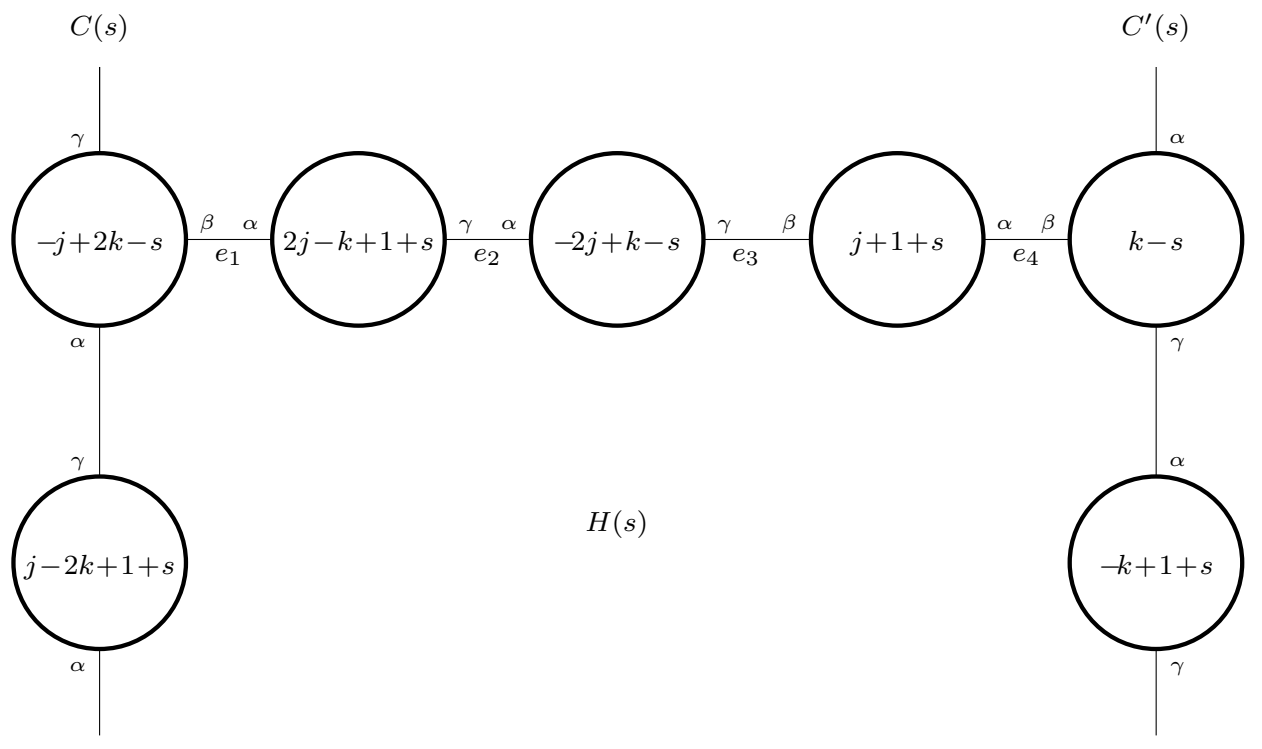

Figure 25 


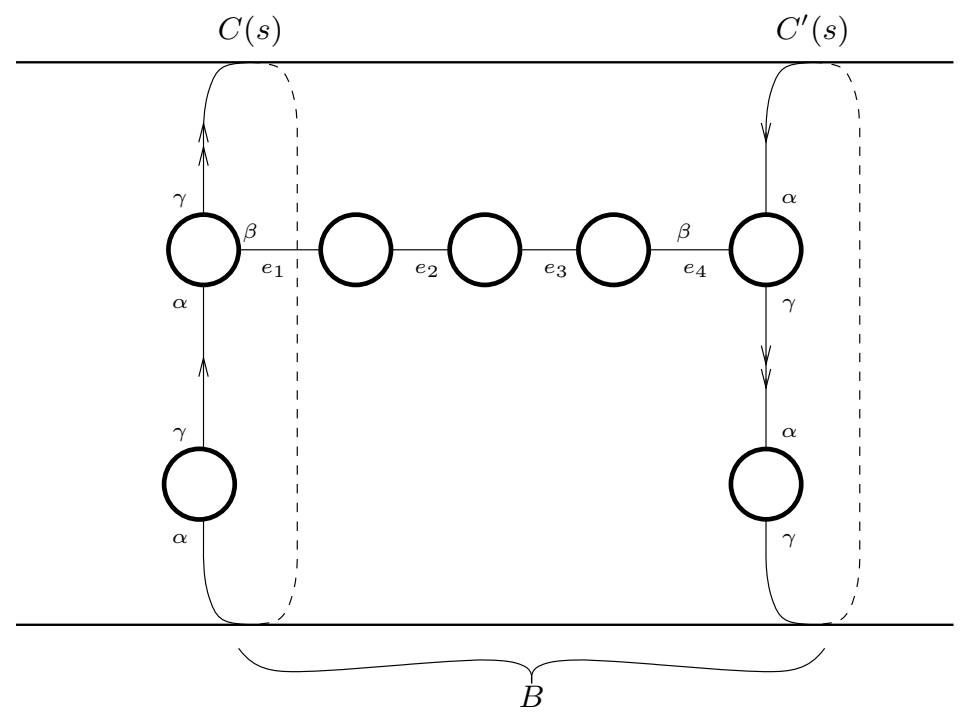

Figure 26

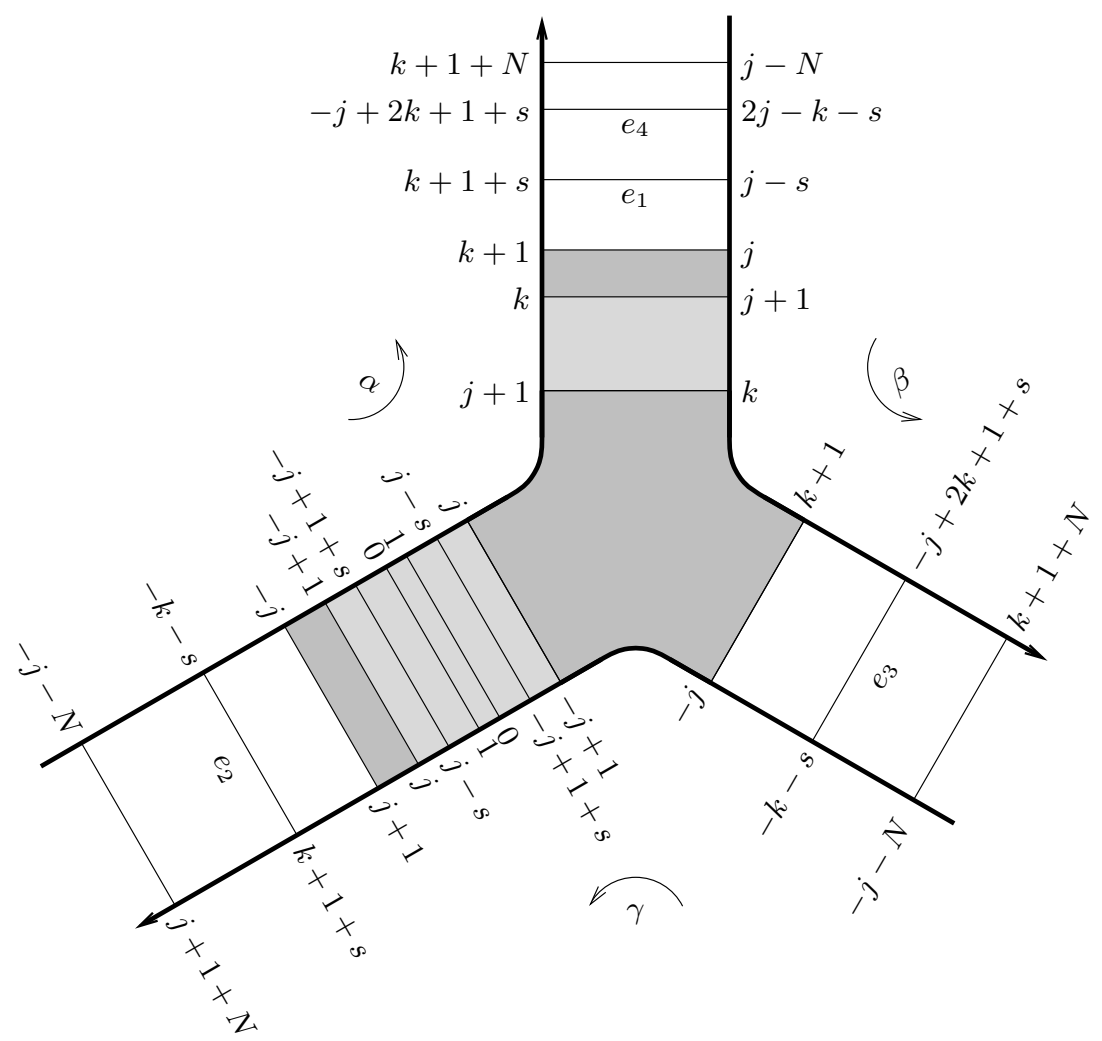

Figure 27 
Claim 6.15. Let $T=T(j, k, N)$ corresponding to $\sigma \in \Sigma_{\text {II }}$ be of Type II such that $N>k-j$ and $2 j-k>0$. Let $\epsilon, \epsilon^{\prime}$ be the extended Scharlemann cycles for $\sigma$ with corners $\overline{-j+1, j}, \overline{j+1, k}$ (resp.) in $T(j, k, N)$. For each

$$
0 \leq s \leq \min \{N-(k-j), j-1\}
$$

there is a configuration $H(s)$ in $\widehat{F}$ consisting of disjoint curves $C(s), C^{\prime}(s)$ in $a(\epsilon)$ connected by a path of four edges of $\sigma$. Furthermore:

(1) If $C\left(s_{1}\right)$ shares vertices with $H\left(s_{2}\right), s_{1} \neq s_{2}$, then $C\left(s_{1}\right)=C^{\prime}\left(s_{2}\right)$ and $C\left(s_{1}\right), H\left(s_{2}\right)$ share no other vertices or edges. In particular,

$$
\mathcal{B}_{\sigma}=\{C(s) \mid 0 \leq s \leq \min \{N-(k-j), j-1\}\}
$$

is a collection of $\min \{N-(k-j)+1, j\}$ disjoint curves. Thus

$$
\left|\mathcal{B}_{\sigma}\right|= \begin{cases}N-(k-j)+1 & \text { if } \sigma \in(\mathbb{I I}, 3), \\ j & \text { if } \sigma \in(\mathbb{I}, 4) \cup(\mathbb{I}, 5) .\end{cases}
$$

(2) Each vertex of $G_{F}$ lies in at most one element of $\mathcal{B}_{\sigma}$.

(3) If $H(s)$ shares a vertex with an element of $a(\epsilon)$ or $a\left(\epsilon^{\prime}\right)$, then that element is either $C(s)$ or $C\left(s^{\prime}\right)$.

(4) No $H(s)$ lies in an annulus on $\widehat{F}$.

Proof. We work with $\sigma$ after it is relabelled as $T(j, k, N)$; however, $H(s)$ is taken to be under the original labelling. Note that as $\sigma$ is Type II, $j<k \leq 3 j$. See Figure 27.

For $0 \leq s \leq \min \{N-(k-j), j-1\}$ let $C(s)$ be the essential curve of $a(\epsilon)$ on $\widehat{F}$ formed by the two $(j-s,-j+1+s)$-edges in $\epsilon$ (note that $1 \leq$ $\max \{k-N, 1\} \leq j-s \leq j)$. Let $C^{\prime}(s)$ be the essential curve of $a(\epsilon)$ formed by the two $(2 j-k-s,-2 j+k+s+1)$-edges in $\epsilon$ (note that $-j+1<$ $\max \{1-k+j, j-N\} \leq 2 j-k-s \leq 2 j-k<j\})$. The four edges $e_{1}, e_{2}, e_{3}, e_{4}$ shown in Figure 27, along with $C(s), C^{\prime}(s)$, give a configuration, $H(s)$, in the graph $G_{F}$. The vertices these edges connect are included in $H(s)$. Note that these seven vertices of $H(s)$ are distinct $(k-j$ is even).

Assume $C\left(s_{1}\right)$ shares vertices with $H\left(s_{1}\right), s_{1} \neq s_{2}$. As the vertices of $C\left(s_{1}\right)$ are labelled between $-j+1$ and $j$ and the vertices $j+k+1-\left(j-s_{2}\right), 2 k+1-\left(j-s_{2}\right)$, $-j-k+\left(j-s_{2}\right)$ are outside this range, $C\left(s_{1}\right)$ can only share vertices with $C\left(s_{2}\right)$ or $C^{\prime}\left(s_{2}\right)$. As these are curves of $a(\epsilon)$, either $C\left(s_{1}\right)=C\left(s_{2}\right)$ or $C\left(s_{1}\right)=C^{\prime}\left(s_{2}\right)$. As $1 \leq j-s \leq j$ for each $s$, it must be that $C\left(s_{1}\right)=C^{\prime}\left(s_{2}\right)$ (corresponding to $s_{2}-s_{1}=j-k$ or $\left.s_{2}+s_{1}=3 j-k-1\right)$. This proves parts (1) and (2) of the claim.

For parts (3) and (4), we argue as for (3) and (4) of Claim 6.14,

Lemma 6.16. For $\sigma \notin \bigcup_{i=\mathrm{I}, \mathbb{I} ; j=3,4,5} \Sigma_{\mathbb{I}}(i, j)$ define $\mathcal{B}_{\sigma}=\emptyset$. Then:

$$
\sum_{\sigma \in \Sigma_{\mathbb{I I}}}\left|\mathcal{B}_{\sigma}\right| \leq 24(g-1)
$$

Proof. Consider the collection $\mathcal{B}$ of all elements of $\mathcal{B}_{\sigma}$ taken (without equating elements) over all $\sigma \in \Sigma_{\mathrm{II}}$ (and hence over all $\sigma \in \bigcup_{i=\mathrm{I}, \mathbb{I} ; j=3,4,5} \Sigma_{\mathrm{II}}(i, j)$ ). By Remark 6.8, each vertex of $G_{F}$ is a label in at most two elements of $\Sigma_{\mathrm{II}}$. On the other hand, for a fixed $\sigma$ a vertex of $G_{F}$ lies in at most one element of $\mathcal{B}_{\sigma}$. Thus any vertex belongs to at most two elements of $\mathcal{B}$. 
Partition $\mathcal{B}$ as $\mathcal{B}_{0} \cup \mathcal{B}_{1} \cup \mathcal{B}_{2}$ as in the proof of Lemma 6.13. Again as in the proof of that lemma, we get disjoint collections of curves $\mathcal{E}$ and $\mathcal{F}$, where no element of $\mathcal{E}$ is parallel on $\widehat{F}$ to either another element of $\mathcal{E}$ or an element of $\mathcal{F}$, and any pair of parallel elements of $\mathcal{F}$ are (before perturbation) disjoint.

We claim that at most four elements of $\mathcal{F}$ can be parallel on $\widehat{F}$. For suppose we had five such parallel curves. They all come from extended Scharlemann cycles of length 2 within elements of $\Sigma_{\mathbb{I I}}$. Consequently, these five parallel, disjoint curves must arise from at most two different elements of $\bigcup_{i=\mathrm{I}, \mathrm{I} ; j=3,4,5} \Sigma_{\mathrm{II}}(i, j)$. Otherwise, by Corollary 5.15, the maximal extended Scharlemann cycles in each of three different elements $\sigma_{1}, \sigma_{2}, \sigma_{3}$ of $\bigcup_{i=\mathrm{I}, \mathrm{I} ; j=3,4,5} \Sigma_{\mathbb{I}}(i, j)$ must have the same core Scharlemann cycles. This would imply that the ideal corners of these elements would overlap, contradicting the minimality of $\Sigma_{\mathrm{II}}$ (see Remark 6.8).

Thus three of these five parallel curves, $c_{1}, c_{2}, c_{3}$, all belong to $\mathcal{B}_{\sigma}$ for a single $\sigma \in \bigcup_{i=\mathrm{I}, \mathrm{I} ; j=3,4,5} \Sigma_{\mathrm{II}}(i, j)$. They all belong to the same extended Scharlemann cycle in $\sigma$. Take $c_{2}$ to be between $c_{1}, c_{3}$. Then $c_{2} \subset H(s)$ coming from $\sigma$ as described in Claims 6.14 and 6.15. If $H(s)$ is disjoint from both $c_{1}$ and $c_{3}$, then $H(s)$ lies in the annulus between $c_{1}, c_{3}$, contradicting these claims. So we may assume that $H(s)$ intersects, say, $c_{1}$. But then, again by these claims, $c_{1}, c_{2}$ must form the curves $C(s), C^{\prime}(s)$ of $H(s)$. (In these claims, if $H(s)$ intersects an element of an extended Scharlemann cycle in $\sigma$, then that element is $C(s)$ or $C^{\prime}(s)$.) Then $H(s)$ lies in the annulus between $c_{1}, c_{2}$, contradicting these claims. (If $H(s)$ intersected $c_{3}$ the same argument applied to $c_{3}$ would equate it with $c_{1}$.)

We thus get a subcollection $\mathcal{F}^{\prime}$ of $\mathcal{F}$ such that no two elements of $\mathcal{F}^{\prime}$ are parallel and $\left|\mathcal{F}^{\prime}\right| \geq|\mathcal{F}| / 4$. Therefore $|\mathcal{E}|+\left|\mathcal{F}^{\prime}\right| \leq 3(g-1)$. Hence

$$
\begin{aligned}
24(g-1) & \geq 8|\mathcal{E}|+8\left|\mathcal{F}^{\prime}\right| \\
& \geq 2\left|\mathcal{D}_{1}^{(1)}\right|+2|\mathcal{F}| \\
& =2\left|\mathcal{B}_{0}\right|+2\left|\mathcal{B}_{1}\right|+\left|\mathcal{B}_{2}\right| \\
& \geq|\mathcal{B}|,
\end{aligned}
$$

as claimed.

Remark 6.17. Lemmas 6.13 and 6.16 linearly bound $\sum N_{\sigma}$, where this sum is taken over $\Sigma_{\mathrm{II}}-((\mathrm{I}, 5) \cup(\mathrm{I}, 6) \cup(\mathrm{I}, 5) \cup(\mathrm{I}, 6))$. These bounds come from the collections $\mathcal{C}_{\sigma}$ and $\mathcal{B}_{\sigma}$. In a similar way, the following collections $\mathcal{A}_{\sigma}$ will be used to linearly bound $\sum N_{\sigma}$, where the sum is taken over the remaining $(\mathrm{I}, 5) \cup(\mathrm{I}, 6) \cup(\mathrm{I}, 5) \cup(\mathrm{I}, 6)$.

Claim 6.18. Let $T(j, k, N)$ corresponding to $\sigma \in \Sigma_{\text {II }}$ be of Type I such that $N>$ $4 j-2 k-1$ (so that $\sigma \in(\mathrm{I}, 5) \cup(\mathrm{I}, 6)$ ). For each $0 \leq s \leq N-2 j$ there is an essential curve $\mathfrak{a}(s)$ in $\widehat{F}$ consisting of 6 distinct edges and vertices. Furthermore, $\mathfrak{a}\left(s_{1}\right)$ intersects $\mathfrak{a}\left(s_{2}\right), s_{2}>s_{1}$, if and only if $s_{2}-s_{1}$ is an element of $\{j-k, 2 j, k+j\}$. Indeed, assume $s_{2}-s_{1}$ is an element of $\{j-k, 2 j, k+j\}$. Either

(1) $k \neq 0$ and $\mathfrak{a}\left(s_{1}\right), \mathfrak{a}\left(s_{2}\right)$ share exactly two vertices and a single edge spanning them. After perturbing to be transverse along their common edge, $\mathfrak{a}\left(s_{1}\right)$ and $\mathfrak{a}\left(s_{2}\right)$ intersect transversely once. Or

(2) $k=0$ and $\mathfrak{a}\left(s_{1}\right), \mathfrak{a}\left(s_{2}\right)$ intersect in exactly two edges and the four vertices they connect. In this case $\mathfrak{a}\left(s_{1}\right), \mathfrak{a}\left(s_{2}\right)$ can be perturbed along these edges so that they are transverse and intersect algebraically, geometrically twice. 


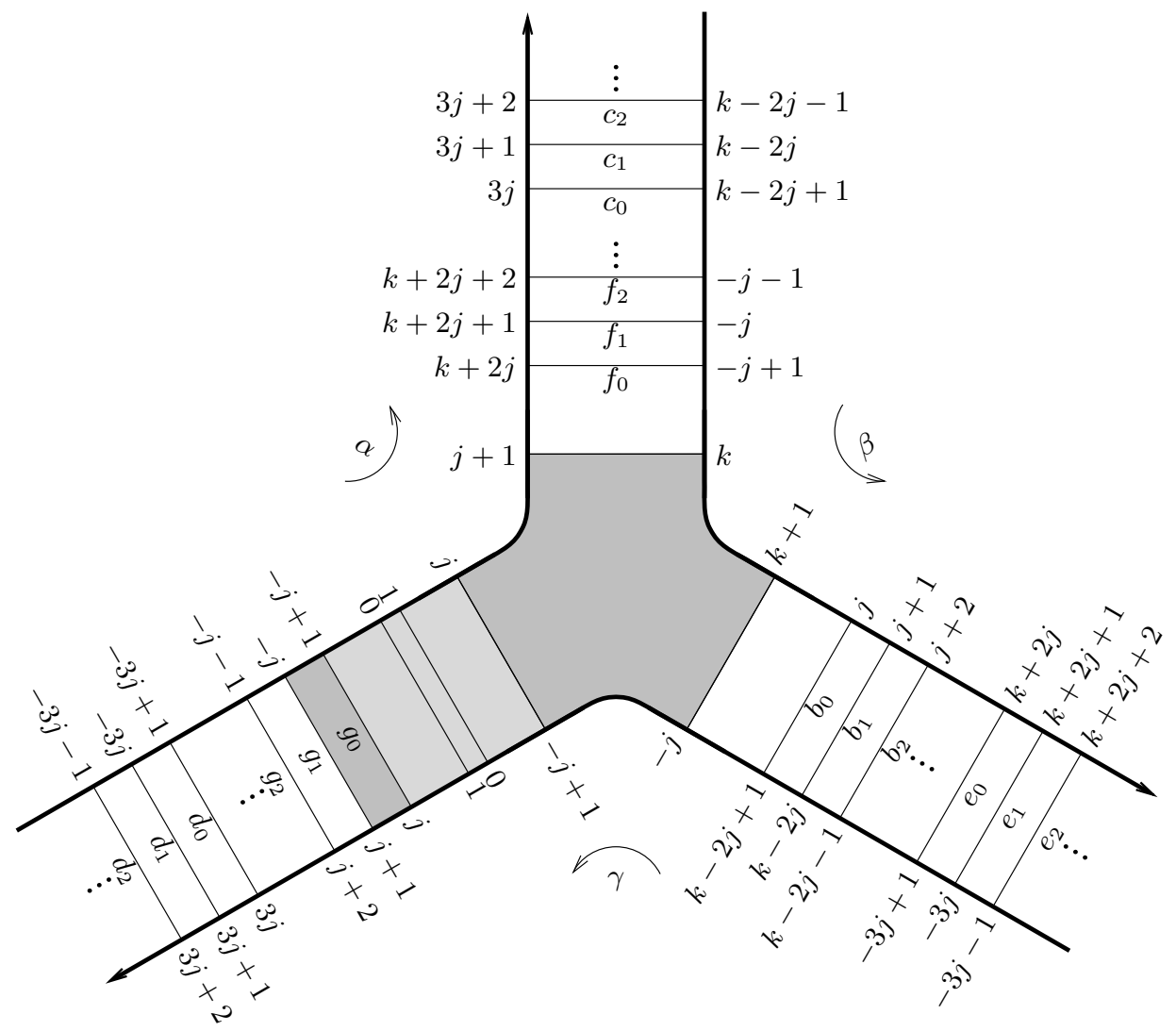

FIGURE 28

As $N-2 j+1 \geq 2(j-k)$, for each $s$ there is an $s^{\prime}$ such that $\left|s-s^{\prime}\right|=j-k$. In particular, each $\mathfrak{a}(s)$ is essential in $\widehat{F}$.

Define $\mathcal{A}_{\sigma}=\{\mathfrak{a}(s) \mid 1 \leq s \leq N-2 j\}$. Then the elements of $\mathcal{B}_{\sigma}$ are disjoint from the elements of $\mathcal{A}_{\sigma}$. Furthermore, each vertex of $G_{F}$ belongs to at most two elements of $\mathcal{A}_{\sigma}$.

Proof. We work with $\sigma$ after it is relabelled as $T=T(j, k, N)$; however, $\mathfrak{a}(s)$ is taken to be under the original labelling.

$T$ is shown in Figure 28. Let $b_{0}, c_{0}, d_{0}, e_{0}, f_{0}$, and $g_{0}$ be the edges pictured in Figure 28, Let $\mathfrak{a}(0)$ be the curve obtained by taking their union. For $0 \leq s \leq N-2 j$ we may take the corresponding edges $b_{s}, c_{s}, d_{s}, e_{s}, f_{s}$, and $g_{s}$ as we move out the arms of $T$ and form the curve $\mathfrak{a}(s)$ by taking their union. Thus we obtain a sequence of curves $\mathfrak{a}(0), \ldots, \mathfrak{a}(N-2 j)$ on $\widehat{F}$. Note that $N \geq 2(j+(j-k))-1>2 j$.

If $\mathfrak{a}\left(s_{1}\right)$ and $\mathfrak{a}\left(s_{2}\right)$ intersect on the Heegaard surface, then they intersect in vertices and possibly coincide along edges.

The labels of the vertices of $\mathfrak{a}(s)$ are $3 j+s, k+2 j+s, j+s,-j+1-s, k-2 j+1-s$, and $-3 j+1-s$ in decreasing order. Each of these vertices is different, so $\mathfrak{a}(s)$ is embedded. Recall $N-2 j \geq s \geq 0$. As no label appears twice along a corner of $T$, 


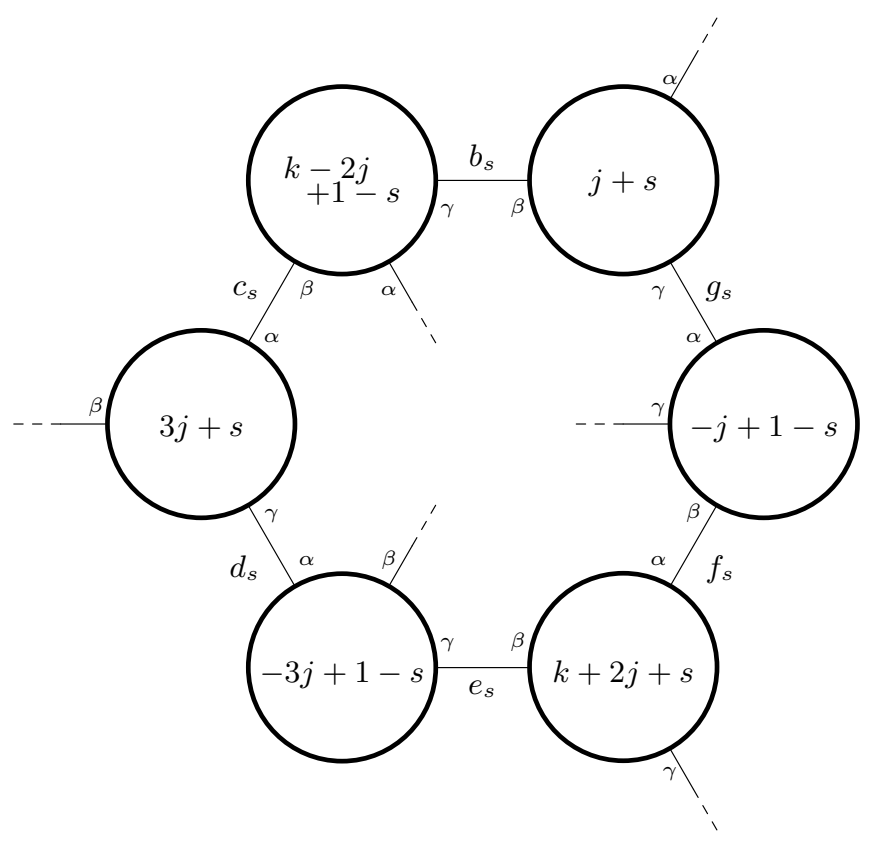

FigURE 29

the vertices of $\mathfrak{a}\left(s_{1}\right)$ and $\mathfrak{a}\left(s_{2}\right)$ overlap if and only if:

(1) $s_{2}-s_{1}=j-k ; k+2 j+s_{2}=3 j+s_{1}$ and $-j-s_{2}+1=k-2 j-s_{1}+1$,

(2) $s_{2}-s_{1}=2 j ; j+s_{2}=3 j+s_{1}$ and $-j-s_{2}+1=-3 j-s_{1}+1$,

(3) $s_{2}-s_{1}=j+k ; j+s_{2}=k+2 j+s_{1}$ and $k-2 j-s_{2}+1=-3 j-s_{1}+1$.

Figure 29 shows $\mathfrak{a}(s)$ as it lies on $\widehat{F}$. If $\mathfrak{a}\left(s_{1}\right), \mathfrak{a}\left(s_{2}\right)$ intersect, then they agree exactly along a single edge (note in Figure 28 that the sum of the labels at an edge determines the arm in which the edge lies, and no label appears twice along a corner of $T$ ). If $k \neq 0$, then $\mathfrak{a}\left(s_{1}\right)$ and $\mathfrak{a}\left(s_{2}\right)$ can be perturbed so that they intersect exactly once (the $\alpha, \beta, \gamma$ labels that are not labels of edges of $\mathfrak{a}\left(s_{1}\right)$, say, alternately appear on one side or the other of $\left.\mathfrak{a}\left(s_{1}\right)\right)$. If $k=0$, then (1) and (3) both hold $\left(e_{s_{1}}=b_{s_{2}}\right.$ and $\left.f_{s_{2}}=c_{s_{1}}\right)$, and one similarly checks that $\mathfrak{a}\left(s_{1}\right), \mathfrak{a}\left(s_{2}\right)$ intersect algebraically and geometrically twice after perturbation. In any case, each vertex of $G_{F}$ belongs to at most two elements of $\mathcal{A}_{\sigma}$.

The edges constituting elements of $\mathcal{B}_{\sigma}$ come from the extended Scharlemann cycle of $\sigma$, while the edges of an element of $\mathcal{A}_{\sigma}$ lie outside the extended Scharlemann cycle. (Note $\mathfrak{a}(0) \notin \mathcal{A}_{\sigma}$.) This verifies the last sentence of the claim.

Claim 6.19. Let $T(j, k, N)$ corresponding to $\sigma \in \Sigma_{\mathbb{I}}$ be of Type II such that $N>$ $3 k-j-1$ (so that $\sigma \in(\mathbb{I}, 5) \cup(\mathbb{I}, 6)$ ). For each $0 \leq s \leq N-k-j$ there is an essential curve $\mathfrak{a}(s)$ in $\widehat{F}$ consisting of 6 distinct edges and vertices. Furthermore, $\mathfrak{a}\left(s_{1}\right)$ intersects $\mathfrak{a}\left(s_{2}\right), s_{2}>s_{1}$, if and only if $s_{2}-s_{1}$ is an element of $\{k-j, 2 j, k+j\}$.

Indeed, assume $s_{2}-s_{1}$ is an element of $\{k-j, 2 j, k+j\}$. Either

(1) $k \neq 3 j$ and $\mathfrak{a}\left(s_{1}\right), \mathfrak{a}\left(s_{2}\right)$ share exactly two vertices and a single edge spanning them. Furthermore, after perturbing to be transverse along their common edge, $\mathfrak{a}\left(s_{1}\right)$ and $\mathfrak{a}\left(s_{2}\right)$ intersect transversely once. Or 


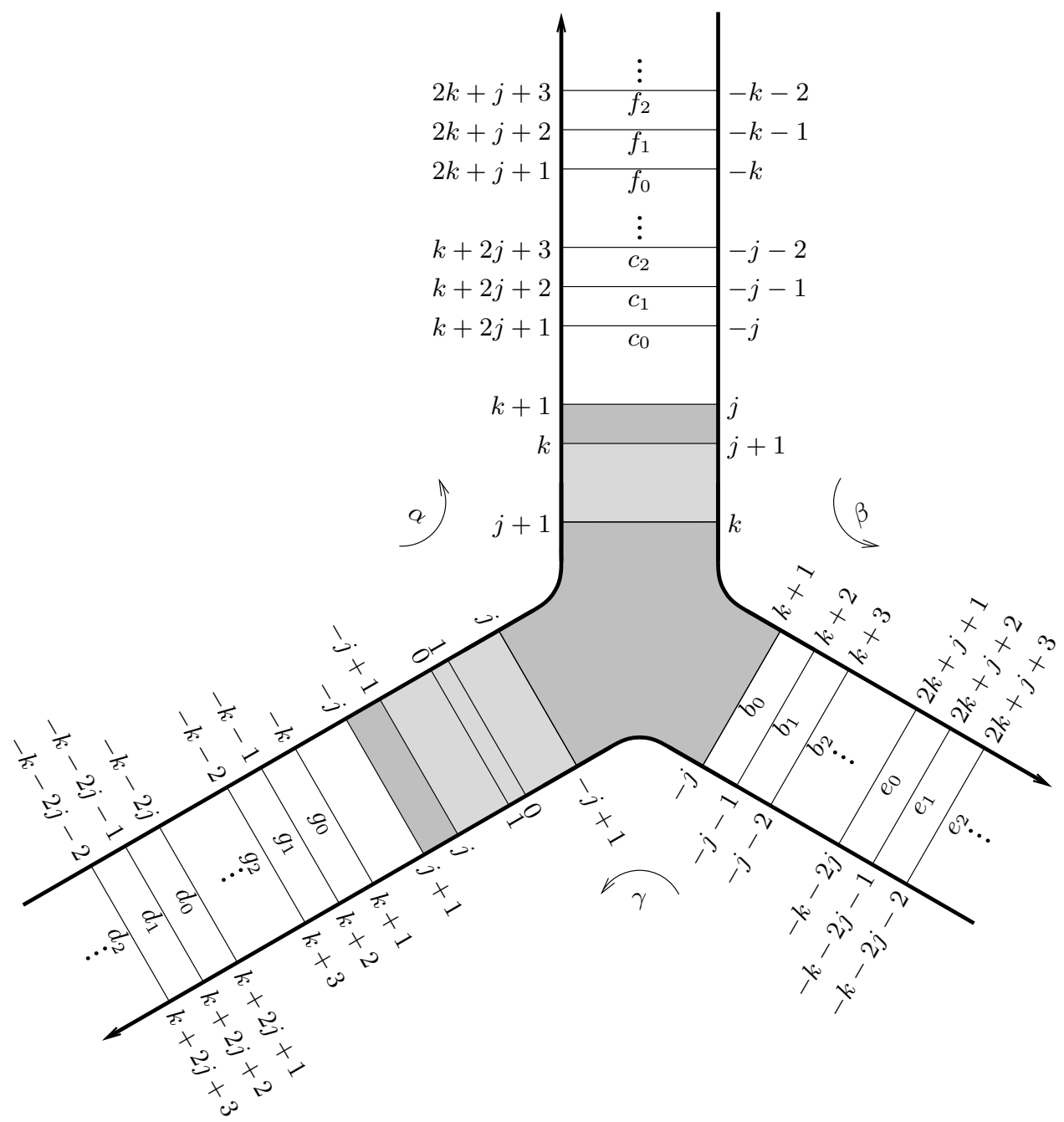

FIGURE 30

(2) $k=3 j$ and $\mathfrak{a}\left(s_{1}\right), \mathfrak{a}\left(s_{2}\right)$ intersect in exactly two edges and the four vertices they connect. In this case $\mathfrak{a}\left(s_{1}\right), \mathfrak{a}\left(s_{2}\right)$ can be perturbed along these edges so that they are transverse and intersect algebraically, geometrically twice.

As $N-j-k+1 \geq 2(k-j)$, for each $s$ there is an $s^{\prime}$ such that $\left|s-s^{\prime}\right|=k-j$. In particular, each $\mathfrak{a}(s)$ is essential in $\widehat{F}$.

Define $\mathcal{A}_{\sigma}=\{\mathfrak{a}(s) \mid 1 \leq s \leq N-k-j\}$. Then the elements of $\mathcal{A}_{\sigma}$ are disjoint from those of $\mathcal{B}_{\sigma}$. Furthermore, a vertex of $G_{F}$ belongs to at most two elements of $\mathcal{A}_{\sigma}$.

Proof. We work with $\sigma$ after it is relabelled as $T=T(j, k, N)$; however, $\mathfrak{a}(s)$ is taken to be under the original labelling.

$T$ is shown in Figure [30, Let $b_{0}, c_{0}, d_{0}, e_{0}, f_{0}$, and $g_{0}$ be the edges pictured in Figure [30. Let $\mathfrak{a}(0)$ be the curve obtained by taking their union. For $0 \leq s \leq$ $N-k-j$ we may take the corresponding edges $b_{s}, c_{s}, d_{s}, e_{s}, f_{s}$, and $g_{s}$ as we move 


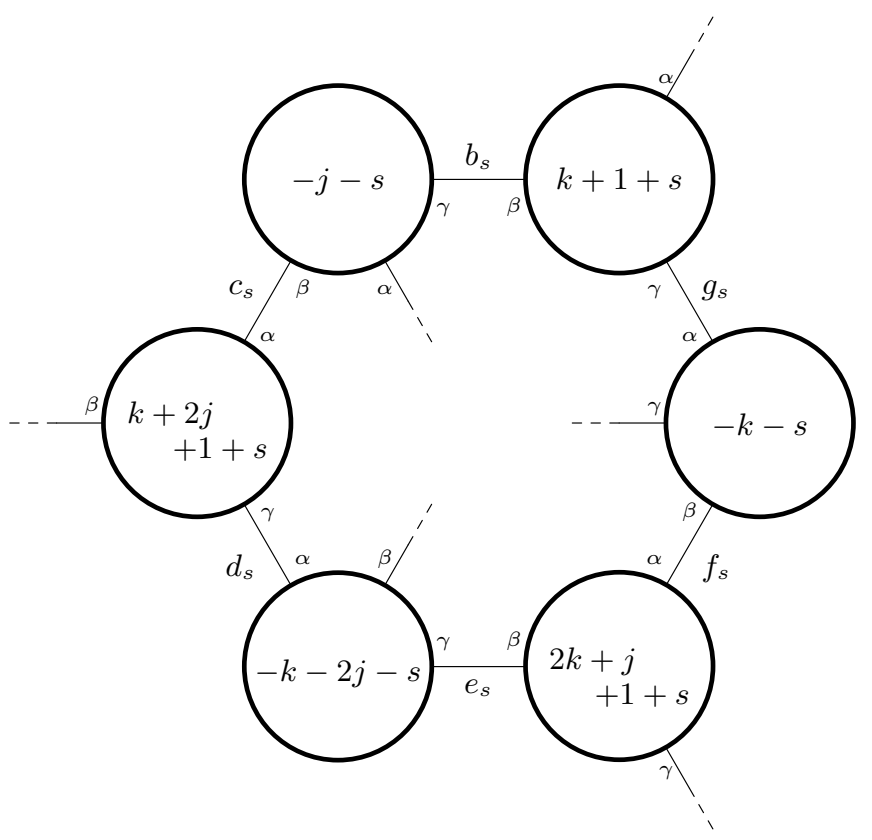

FiguRE 31

out the arms of $T$ and form the curve $\mathfrak{a}(s)$ in $\widehat{F}$ by taking their union. Thus we obtain a sequence of curves $\mathfrak{a}(0), \ldots, \mathfrak{a}(N-k-j)$ on $\widehat{F}$. Note that $N \geq k+j$.

If $\mathfrak{a}\left(s_{1}\right)$ and $\mathfrak{a}\left(s_{2}\right)$ intersect on the Heegaard surface, then they intersect in vertices and possibly coincide along edges.

The vertices of $\mathfrak{a}(s)$ are $2 k+j+1+s, k+2 j+1+s, k+1+s,-j-s,-k-s$, and $-k-2 j-s$. These vertices are distinct and $\mathfrak{a}(s)$ is embedded. Furthermore, the vertices of $\mathfrak{a}\left(s_{1}\right)$ and $\mathfrak{a}\left(s_{2}\right)$ overlap if and only if:

(1) $s_{2}-s_{1}=k-j ; k+2 j+1+s_{2}=2 k+j+1+s_{1}$ and $-j-s_{2}=-k-s_{1}$,

(2) $s_{2}-s_{1}=2 j ; k+1+s_{2}=k+2 j+1+s_{1}$ and $-k-s_{2}=-k-2 j-s_{1}$,

(3) $s_{2}-s_{1}=k+j ; k+1+s_{2}=2 k+j+1+s_{1}$ and $-j-s_{2}=-k-2 j-s_{1}$.

Figure 31 shows $\mathfrak{a}(s)$ as it lies on $\widehat{F}$. As before one sees that if $\mathfrak{a}\left(s_{1}\right), \mathfrak{a}\left(s_{2}\right)$ intersect, then they must coincide along an edge. They can then be perturbed so that they intersect exactly once - except when $k=3 j$ when (1) and (2) both hold. If $k=3 j$ one checks that $\mathfrak{a}\left(s_{1}\right)$ and $\mathfrak{a}\left(s_{2}\right)$ intersect algebraically and geometrically twice after perturbation. In any case, a vertex of $G_{F}$ belongs to at most two elements of $\mathcal{A}_{\sigma}$.

The edges constituting elements of $\mathcal{B}_{\sigma}$ come from the extended Scharlemann cycles of $\sigma$, while the edges of an element of $\mathcal{A}_{\sigma}$ lie outside the extended Scharlemann cycles. This verifies the last sentence of the claim.

Lemma 6.20. For $\sigma \notin \bigcup_{\substack{i=\mathrm{I}, \mathrm{II} \\ j=3,4,5}} \Sigma_{\mathbb{I}}(i, j)$ define $\mathcal{B}_{\sigma}=\emptyset$. For $\sigma \notin \bigcup_{\substack{i=\mathrm{I}, \mathrm{II} \\ j=5,6}} \Sigma_{\mathbb{I}}(i, j)$ define $\mathcal{A}_{\sigma}=\emptyset$. Then:

$$
\sum_{\sigma \in \Sigma_{\mathrm{II}}}\left(\left|\mathcal{A}_{\sigma}\right|+\left|\mathcal{B}_{\sigma}\right|\right) \leq 240(g-1)
$$


Proof. Consider the collection $\mathcal{D}$ of all elements of $\mathcal{A}_{\sigma}, \mathcal{B}_{\sigma}$ taken (without equating elements) over all $\sigma \in \bigcup_{i=1,2 ; j=3,4,5} \Sigma_{\mathbb{I}}(i, j)$. Let $\mathfrak{a}_{\sigma}$ be an element of $\mathcal{A}_{\sigma}$. Then $\mathfrak{a}_{\sigma}$ is disjoint from any element of $\mathcal{B}_{\sigma}$, meets at most three other elements of $\mathcal{A}_{\sigma}$, and each vertex of $\mathfrak{a}_{\sigma}$ belongs to at most one other element of $\mathcal{A}_{\sigma}$. Also, any two elements of $\mathcal{B}_{\sigma}$ are disjoint. By Remark 6.8, each vertex of $G_{F}$ is a label in at most two elements of $\Sigma_{\mathrm{II}}$. It follows that each element of $\mathcal{D}$ meets at most $3+6 \cdot 2=15$ other elements of $\mathcal{D}$, the maximum occurring when an element $\mathfrak{a}_{\sigma} \in \mathcal{A}_{\sigma}$ meets three other elements of $\mathcal{A}_{\sigma}$, and for each vertex $v_{i}$ of $\mathfrak{a}_{\sigma}$ there exists $\sigma_{i} \in \Sigma_{\mathbb{I}}, \sigma_{i} \neq \sigma$, such that $v_{i}$ belongs to two elements of $\mathcal{A}_{\sigma_{i}}, 1 \leq i \leq 6$. That is, we find at least $(1 / 16) \sum_{\sigma \in \Sigma_{\mathrm{II}}}\left(\left|\mathcal{A}_{\sigma}\right|+\left|\mathcal{B}_{\sigma}\right|\right)$ disjoint curves coming from $\mathcal{D}$. We need to show that there are at most $5(3 g-3)$ such curves.

Assume for contradiction that $(1 / 16) \sum_{\sigma \in \Sigma_{\mathrm{II}}}\left(\left|\mathcal{A}_{\sigma}\right|+\left|\mathcal{B}_{\sigma}\right|\right)>5(3 g-3)$. Then there must be a collection $\mathcal{E}$ of six disjoint curves of $\mathcal{D}$ that are isotopic on $\widehat{F}$.

Claim 6.21. No element of $\mathcal{E}$ belongs to $\mathcal{A}_{\sigma}$ for $\sigma \in \Sigma_{\mathbb{I}}$.

Proof. Assume there were an $\mathfrak{a}_{\sigma}(s) \in \mathcal{E}$ for some $\sigma$ and $s$ as in Claims 6.18, 6.19, By the same claims, we can find another element $\mathfrak{a}_{\sigma}\left(s^{\prime}\right)$ coming from the same $\mathcal{A}_{\sigma}$ that shares at least one edge with $\mathfrak{a}_{\sigma}(s)$ and which can be perturbed to intersect $\mathfrak{a}_{\sigma}(s)$ algebraically once or twice. As $\mathfrak{a}_{\sigma}\left(s^{\prime}\right)$ must share at least one vertex with any element of $\mathcal{E}$ that it intersects, one of the five curves of $\mathcal{E}-\left\{\mathfrak{a}_{\sigma}(s)\right\}$ must be disjoint from $\mathfrak{a}_{\sigma}\left(s^{\prime}\right)$. But all of these isotopic curves must have algebraic intersection number at least 1 with $\mathfrak{a}_{\sigma}\left(s^{\prime}\right)$.

Claim 6.22. For some $\sigma \in \Sigma_{\mathrm{II}}$ there are three elements of $\mathcal{E}$ that belong to $\mathcal{B}_{\sigma}$. Furthermore, there is an extended Scharlemann cycle $\epsilon$ in $\sigma$ such that each of these three belong to $a(\epsilon)$.

Proof. By Claim 6.21, all the elements of $\mathcal{E}$ come from $\cup \mathcal{B}_{\sigma}$. Consequently they all come from extended Scharlemann cycles of length 2 within elements of $\Sigma_{\mathbb{I}}$. By Corollary 5.15, these extended Scharlemann cycles must have the same core labels. As each of these core labels will lie in the ideal corners of the corresponding elements of $\Sigma_{\mathbb{I}}$, Remark 6.8 implies that these six parallel, disjoint curves in $\mathcal{E}$ belong to at most two different elements of $\Sigma_{\mathbb{I}}$. Hence three belong to the same $\sigma \in \Sigma_{\mathbb{I}}$. Again by Corollary 5.15 they must belong to the same extended Scharlemann cycle within $\sigma$.

Let $c_{1}, c_{2}, c_{3}$ be three parallel curves of Claim 6.22 with $c_{2}$ between $c_{1}, c_{3}$. Then $c_{2} \subset H(s)$ coming from $\sigma$ as described in Claims 6.14 and 6.15. If $H(s)$ is disjoint from both $c_{1}$ and $c_{3}$, then $H(s)$ lies in the annulus between $c_{1}, c_{3}$, contradicting these claims. So we may assume that $H(s)$ intersects, say, $c_{1}$. But then, again by these claims, $c_{1}, c_{2}$ must form the curves $C(s), C^{\prime}(s)$ of $H(s)$. (In these claims, if $H(s)$ intersects an element of an extended Scharlemann cycle in $\sigma$, then that element is $C(s)$ or $C^{\prime}(s)$.) Then $H(s)$ lies in the annulus between $c_{1}, c_{2}$, contradicting these claims. (If $H(s)$ intersected $c_{3}$ the same argument applied to $c_{3}$ would equate it with $c_{1}$.)

Remark 6.23. After Lemmas 6.13 and 6.16, to get a linear bound on $\sum_{\Sigma_{\mathrm{II}}} N_{\sigma}$ we need only bound $\sum_{(\mathbb{I}, 5),(\mathbb{I}, 6)}\left(N_{\sigma}-2 j_{\sigma}\right)+\sum_{(\mathrm{I}, 5),(\mathrm{I}, 6)}\left(N_{\sigma}-\left(j_{\sigma}+k_{\sigma}\right)\right)$. This can be done as in the proof of Lemma 6.20 using only the collections $\mathcal{A}_{\sigma}$. By including the $\mathcal{B}_{\sigma}$ above, we get a slightly improved bound. 
Theorem 6.24.

$$
\left|\mathcal{L}_{\mathrm{I}}\right| / 2 \leq \sum_{\sigma \in \Sigma_{\mathrm{II}}}\left(N_{\sigma}+1\right) \leq 480(g-1) .
$$

That is, there are at most $960(g-1)$ labels $x$ in $G_{Q}$ such that $\Lambda_{x}$ has a trigon face which is of Type I or Type II but $x \notin L(\Sigma)$.

Proof. The first inequality comes from Definition 6.7, so we focus on the second which follows from:

$$
\begin{aligned}
\sum_{\sigma \in \Sigma_{\mathrm{II}}}\left(N_{\sigma}+1\right) & \leq 6 \sum_{\sigma \in \Sigma_{\mathrm{II}}}\left|\mathcal{C}_{\sigma}\right|+\sum_{\sigma \in \Sigma_{\mathrm{II}}}\left|\mathcal{B}_{\sigma}\right|+\sum_{\sigma \in \Sigma_{\mathrm{II}}}\left(\left|\mathcal{B}_{\sigma}\right|+\left|\mathcal{A}_{\sigma}\right|\right) \\
& \leq 6 \cdot 36(g-1)+24(g-1)+240(g-1) \\
& =480(g-1) .
\end{aligned}
$$

Inequality $(* *)$ comes from Lemma 6.20, Lemma 6.13, and Lemma 6.16. For inequality $(*)$ we take the definitions of $\mathcal{C}_{\sigma}, \mathcal{B}_{\sigma}$, and $\mathcal{A}_{\sigma}$ coming from Claims 6.11, 6.12, 6.14, 6.15, 6.18, and 6.19 along with the stipulations that if $\sigma \notin \bigcup_{i=\mathrm{I}, \mathrm{I} ; j=3,4,5} \Sigma_{\mathrm{II}}(i, j)$, then $\mathcal{B}_{\sigma}=\emptyset$, and if $\sigma \notin \bigcup_{i=\mathrm{I}, \mathrm{II} ; j=5,6} \Sigma_{\mathrm{II}}(i, j)$, then $\mathcal{A}_{\sigma}=\emptyset$. The estimates of Figure 32 verify inequality $(*)$. These estimates arise from the partition of $\Sigma_{\mathrm{II}}$ in Definition 6.9 along with the counts listed in the above claims.

In Figure 32, each quantity on the left hand side of the second column is at least $N_{\sigma}+1$. To see this, note that $j_{\sigma}-k_{\sigma} \geq 2$ for $\sigma \in$ (I) and $k_{\sigma}-j_{\sigma} \geq 2$ for $\sigma \in$ (II), and then further note the upper bounds on $N_{\sigma}$ for $\sigma \in$ (I, 2), (I, 4), (II, 2), (II, 4) given in Definition 6.9.

\section{TYPE III TRIGONS}

This section is primarily dedicated to elucidating the structure of Type III trigons which are cycles. We construct a collection $\Sigma_{\mathrm{mI}}$ of such trigons that captures all the labels $x$ of $\Lambda$ that give rise to Type III trigons (whether $x$-cycles or not) that do not already belong to $L(\Sigma)$.

Recall that a Type III trigon on the label $x$ is a trigon face of $\Lambda_{x}$ which, regarded as a subgraph of $\Lambda$, has a single Scharlemann cycle (of length 2) and whose central trigon face (of $\Lambda$ ) has only two corner types. Figure 33 shows a generic (as we shall see below) Type III $x$-cycle trigon where the central trigon face is in the two corner types $\overline{j, j+1,} \overline{l-1, l}$.

We now define the terms ideal corner, trunk, and gnarl associated to a type III trigon.

Definition 7.1. Let $\sigma$ be a Type III trigon on $x$ which is a cycle (i.e., the edges of $\sigma$ form a cycle in $\Lambda_{x}$ so that, when oriented, the tails have label $x$ ). Let $p, p+1$ be the labels of its core Scharlemann cycle. Let $\epsilon$ be the extended Scharlemann cycle containing the core Scharlemann cycle of $\sigma$ which abuts its central trigon face. There is a label $y$ such that $\sigma$ has exactly two edges, $e_{1}, e_{2}$ with labels $x, y$. One can see that $x+y \equiv p+(p+1)(\bmod t)$. That is, around a vertex of $\Lambda$, the labels $x, y$ are equidistant from labels $p, p+1$. Define the ideal corner of $\sigma$, denoted $c(\sigma)$, to be the label interval on a vertex of $\Lambda$ between labels $x$ and $y$ that contains labels $p, p+1$. These edges $e_{1}, e_{2}$ lie on different arms, $a_{1}, a_{2}$ of $\sigma$. The ideal corner $c(\sigma)$ appears twice as an actual corner of $\sigma$ in $\Lambda$ - once between $e_{1}, e_{2}$. For any pair of labels $i, j$ in $c(\sigma)$ such that $i+j \equiv 2 p+1(\bmod t)$, there are exactly two edges of $\Lambda$ in $\sigma$ with endpoints $i, j$. These edges lie in the arms $a_{1}, a_{2}$ of $\sigma$. The subgraph 


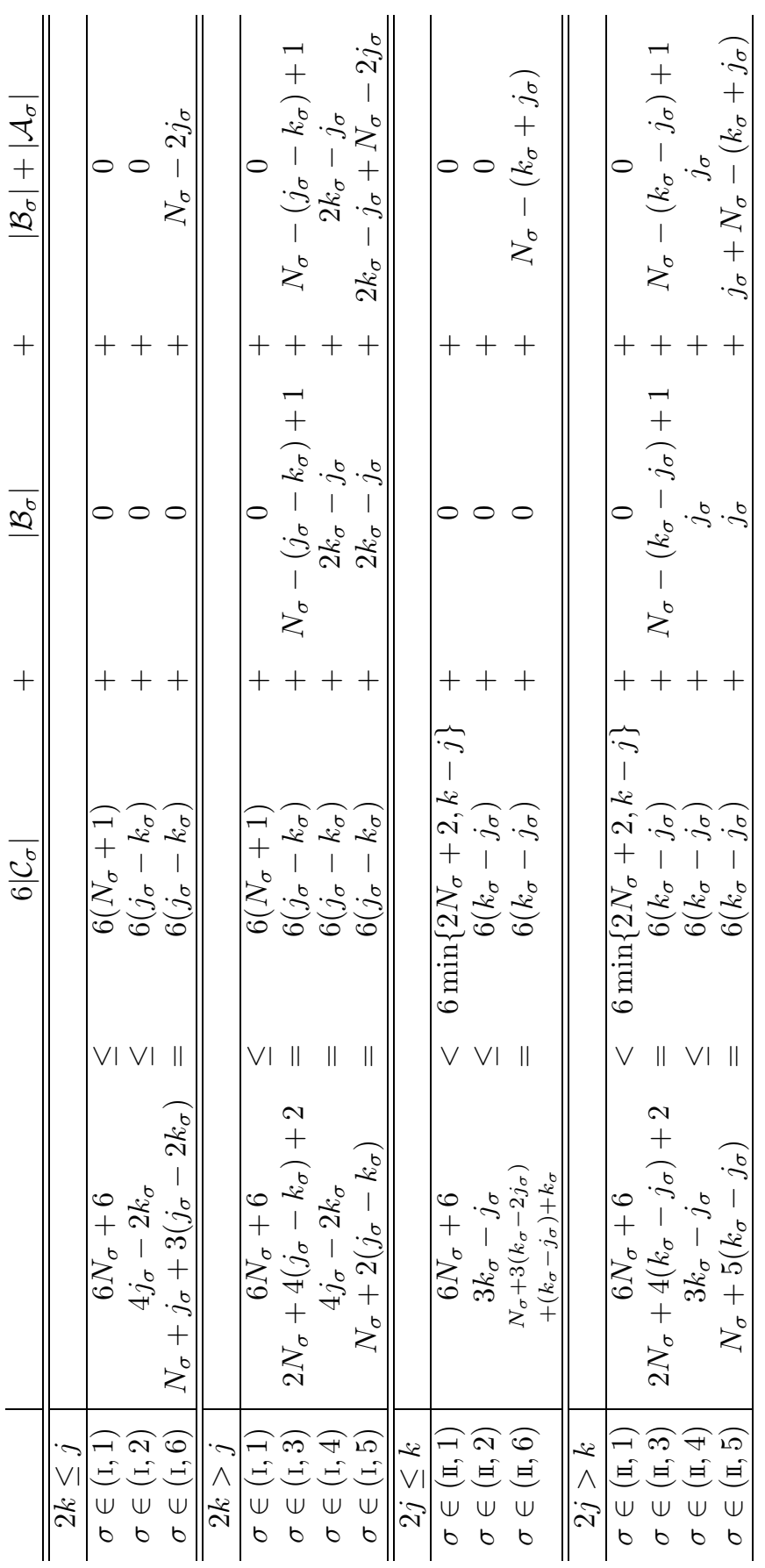

FiguRE 32 


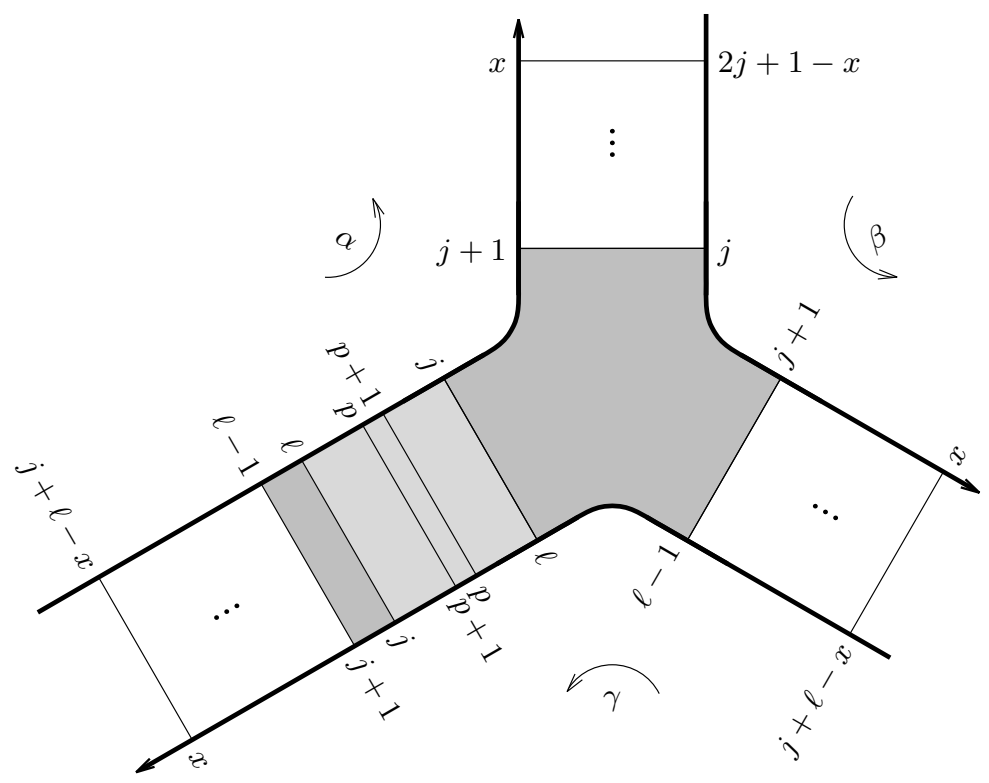

FiguRE 33

of $G_{F}$ formed by the vertices $i, j$ and these two edges is a circle in $\widehat{F}$. If it is an element of $a(\epsilon)$ we call it a trunk of $\sigma$; otherwise we refer to it as a gnarl of $\sigma$ (see also Definition (7.6). To sum up, the ideal corner $c(\sigma)$ has the following properties:

- Every label in $c(\sigma)$ belongs to a unique gnarl or trunk of $\sigma$.

- $x$ is a label in $c(\sigma)$.

- $c(\sigma)$ is symmetric about the consecutive labels $p, p+1$ of the core of $\sigma$.

As a subgraph of $\Lambda$, two of the actual corners of $\sigma$ are the same as $c(\sigma)$. The other either properly contains $c(\sigma)$ or is properly contained in it. After paring down $\sigma$, we may assume this corner is properly contained in $c(\sigma)$. Note that the Type III trigon resulting from this paring down has the same ideal corner but is now a cycle trigon in $y$ rather than $x$.

Definition 7.2. Let $\mathcal{L}_{\mathrm{II}}$ be the collection of labels of $G_{Q}$ that are in the ideal corners of cycle trigons but are not the labels of extended Scharlemann cycles of length 2,3 . That is, $\mathcal{L}_{\mathrm{II}}=\{x \in c(\sigma) \mid \sigma$ is a cycle trigon of Type $\mathrm{III}\}-L(\Sigma)$. Let $\Sigma_{\mathrm{II}}$ be a collection of smallest cardinality of Type III cycle trigons with the property that $\mathcal{L}_{\mathrm{II}} \subset \bigcup_{\sigma \in \Sigma_{\mathrm{II}}} c(\sigma)$. We may assume that each element, $\sigma$, of $\Sigma_{\mathrm{III}}$ has been pared down so that each actual corner of $\sigma$ is a subset of its ideal corner.

\section{Remark 7.3.}

(1) For every $x \in \mathcal{L}-L(\Sigma)$ such that $\Lambda_{x}$ has a trigon which is a Type III non-cycle trigon, there is a $\sigma \in \Sigma_{\mathrm{mI}}$ with $x \in c(\sigma)$, by Lemma 7.4 below.

(2) Item (1) and the definition of $\Sigma_{\mathrm{II}}$ imply: The ideal corners of elements of $\Sigma_{\text {III }}$ include all labels, $x$, of $\mathcal{L}-L(\Sigma)$ such that $\Lambda_{x}$ has a Type III trigon.

(3) The elements of $\Sigma_{\mathrm{II}}$ have different core Scharlemann cycles (by the minimality of $\Sigma_{\mathbb{I I}}$, because the ideal corners are symmetric about the core labels). 


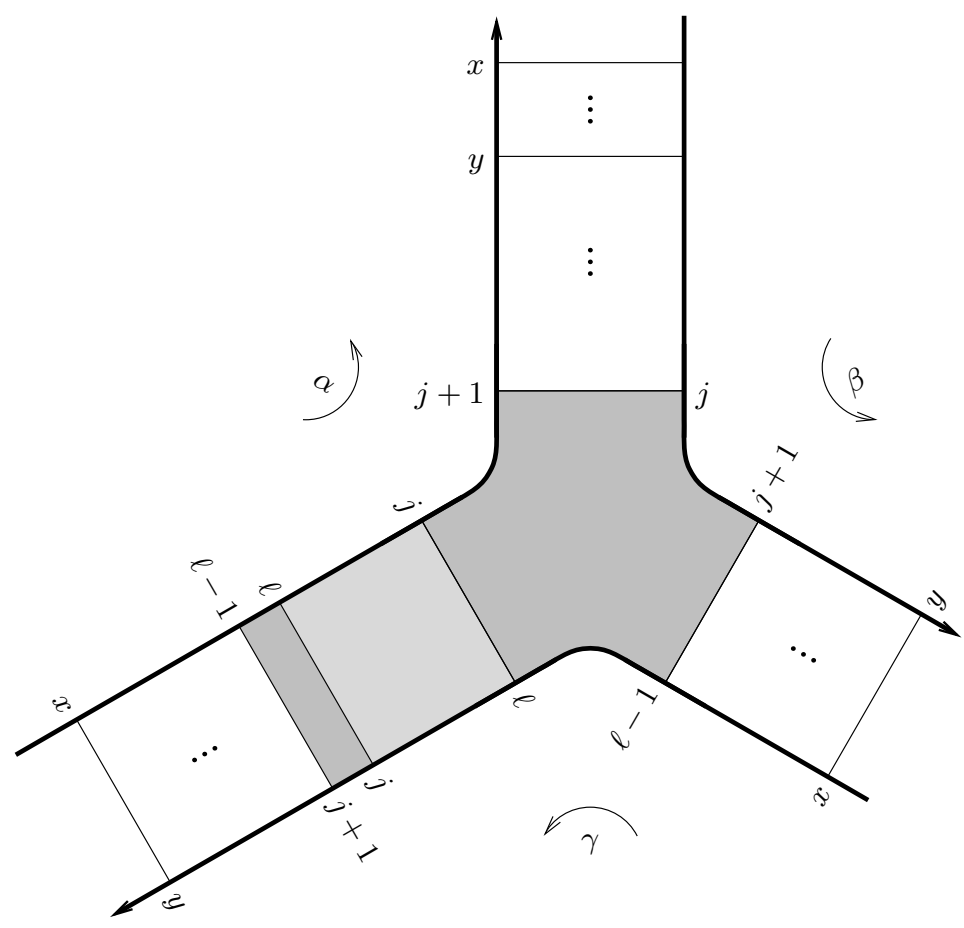

FIGURE 34

As different core Scharlemann cycles cannot lie on a common annulus in $\widehat{F}$ (else there is a Klein bottle), we can construct a family of $\left|\Sigma_{\mathrm{II}}\right|$ different curves on $\widehat{F}$ that satisfy Property $P(2)$. Hence $\left|\Sigma_{\mathrm{III}}\right| \leq F_{2}(g)$, by Lemma 3.2 .

(4) Note that $\mathcal{L}_{\mathrm{II}}$ and $\mathcal{L}_{\mathrm{III}}$ are not necessarily disjoint. Also note that every element of $\mathcal{L}_{\mathrm{III}}$ corresponds to the vertex of a gnarl.

Lemma 7.4. Let $x \in \mathcal{L}-L(\Sigma)$. If there is a Type III $x$-trigon of $\Lambda$ that is not an $x$-cycle, then there is a trigon $\sigma \in \Sigma_{\mathrm{II}}$ such that $x \in c(\sigma)$.

Proof. The $x$-trigon has one corner, $\alpha$, with both endpoints labelled $x$. It has a corner, $\beta$, with no label $x$, and a corner, $\gamma$, with exactly one endpoint labelled $x$. Let $j, j+1$ be the labels of the central trigon face at $\alpha$. Let $\ell-1, \ell$ be the other labels of this central trigon, such that $j, \ell$ have opposite parity. Two of the edges of the $x$-trigon will have labels $x, y$ where $x+y \equiv j+\ell(\bmod t)$. Using the fact that no other labels $x$ appear on $\alpha, \beta, \gamma$, one sees that, up to whether the labels increase or decrease as you move clockwise along these corners, the $x$-trigon appears as in Figure 34. Inside this $x$-trigon is the pictured $y$-cycle, forming a Type III cycle trigon. The ideal corner of this Type III $y$-cycle trigon contains $x$ (it is given by $\gamma$ in the figure). By definition, there is a $\sigma \in \Sigma_{\mathrm{II}}$ such that $c(\sigma)$ includes all elements in this corner.

Definition 7.5. Let $\sigma$ be a Type III cycle trigon. If $u, v$ are labels in $c(\sigma)$, then $\overline{u v}$ is the interval of labels between them contained in $c(\sigma)$. 

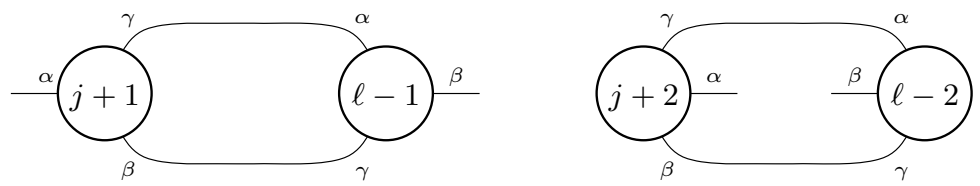

FiguRE 35

Let $\sigma \in \Sigma_{\mathrm{II}}$ be as above. Then up to the ordering of the labels around a corner (i.e., whether they increase as you move clockwise or anti-clockwise around a corner) and rechoosing which label is called $x$, we may assume $\sigma$ appears as in Figure 33 . The core Scharlemann cycle of $\sigma$ has labels $p, p+1$ where $p=(j+\ell-1) / 2$. There $c(\sigma)$ occurs along the corners labelled $\alpha, \gamma$. The shorter corner is labelled $\beta$. The lower arms in the figure are the arms $a_{1}, a_{2}$. Pair the vertices in the disjoint intervals $\overline{j+\ell-x, \ell-1}$ and $\overline{j+1, x}$. Each such pair of vertices has two edges in $\sigma$ that forms a gnarl on $\widehat{F}$. See Figure 35 .

Property 1. The $\alpha, \beta$ labels at the ends of edges of $\sigma \in \Sigma_{\mathrm{III}}$ (Figure 33) incident to a gnarl of $\sigma$ (but not belonging to a gnarl of $\sigma$ ) lie on the same side of the gnarl in $\widehat{F}$. See Figure 35. Specifically, let $r, s$ be the labels in $\sigma$ of two edges $e_{1}, e_{2}$ forming a gnarl, with $r$ the label at corner $\alpha$. There is another edge, $e_{3}$, in $\sigma$ incident to the $\alpha$-corner with label $s$. Assume there is another edge, $e_{4}$, in $\sigma$ with label $r$ at the $\beta$-corner. Consider these edges in $\widehat{F}$. Then $e_{3}, e_{4}$ lie on the same side in $\widehat{F}$ of the gnarl $e_{1} \cup e_{2}$. (In Figure 35, $\{r, s\}$ is $\{j+1, l-1\}$ and $\{j+2, l-2\}$.)

Set $w=j-\ell+1$. We separate the edges in the upper arm of $\sigma$ into $\bmod w$ classes: Let $n$ be such that

$$
(1+j)+n w \leq x \leq j+(n+1) w=(\ell-1)+(n+2) w
$$

Definition 7.6. Let $r$ be such that $(j+\ell+1) / 2=p+1 \leq r \leq j$. Note that $p=(j+\ell-1) / 2 \geq j+\ell-r \geq \ell$. We may now label $\sigma$ as in Figure 36 .

For each $r$ we see in $\widehat{F}$ the component of $\sigma$ pictured in Figure 37. Note that a gnarl in this component may or may not be essential on $\widehat{F}$ - if essential we call it a wrapping gnarl, if inessential we call it a simple gnarl. The interior of a simple gnarl is the interior of the disk it bounds on $\widehat{F}$. Recall that $\epsilon$ is the extended Scharlemann cycle in $\sigma$. The component of $\sigma$ (a subset of $\widehat{F}$ ) corresponding to $r$ consists of a curve of $a(\epsilon)$ between the vertices with labels $r, \ell+j-r$, called the trunk of the component, along with two branches, $r,(\ell+j-r)+w, r-w,(\ell+j-r)+2 w, \ldots$ and $\ell+j-r, r+w,(\ell+j-r)-w, r+2 w, \ldots$. We think of a branch of the component of $\sigma$ as a path of gnarls in $\widehat{F}$. We orient the branch by labelling its vertices $0,1,2, \ldots$ as pictured on the left of Figure 38 (which shows this labeling for the two branches of a component). The right side of that figure pictures the corresponding labelling on the trigon $\sigma$. If $\mathfrak{B}$ is a branch of $\sigma$ and $x$ is a vertex of this branch, let $b_{\mathfrak{B}}(x) \in\{0,1,2, \ldots\}$ be the label coming from the oriented $\mathfrak{B}$ (e.g. $b_{\mathfrak{B}_{1}}(r)=0, b_{\mathfrak{B}_{1}}((l+j-r)+w)=1$ for $\mathfrak{B}_{1}$ the first branch described above.) 
$w=j-\ell+1$

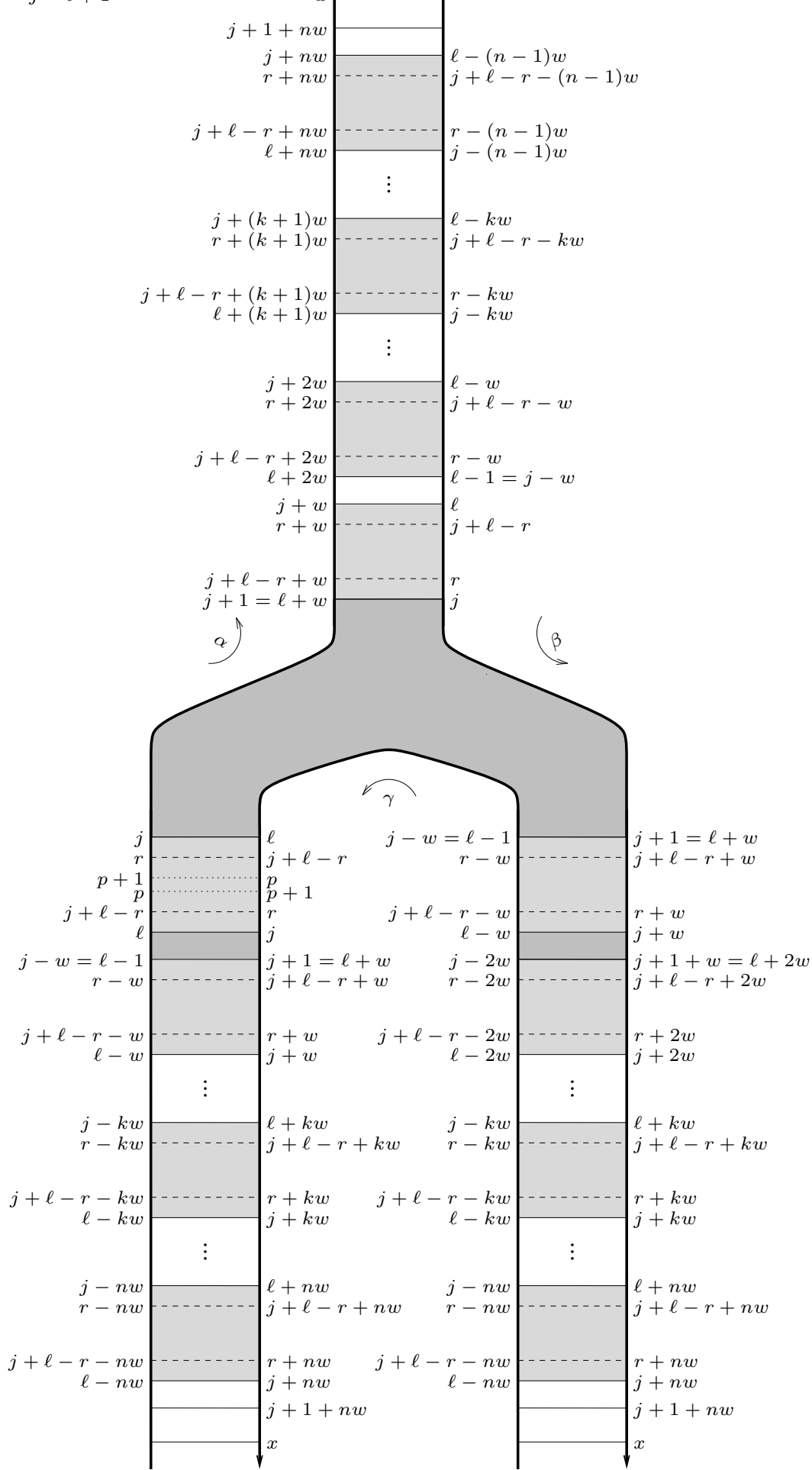

FiguRE 36 

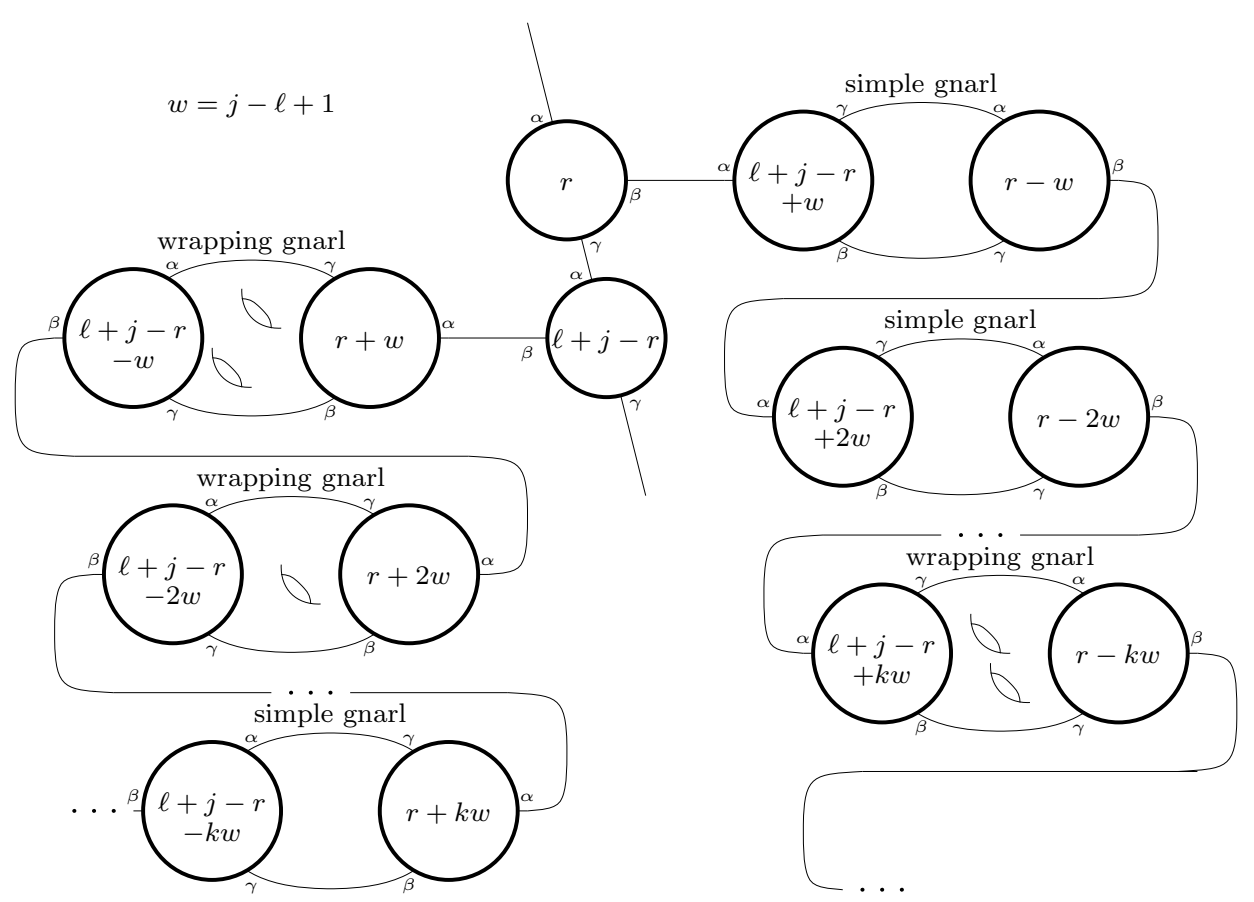

FiguRE 37

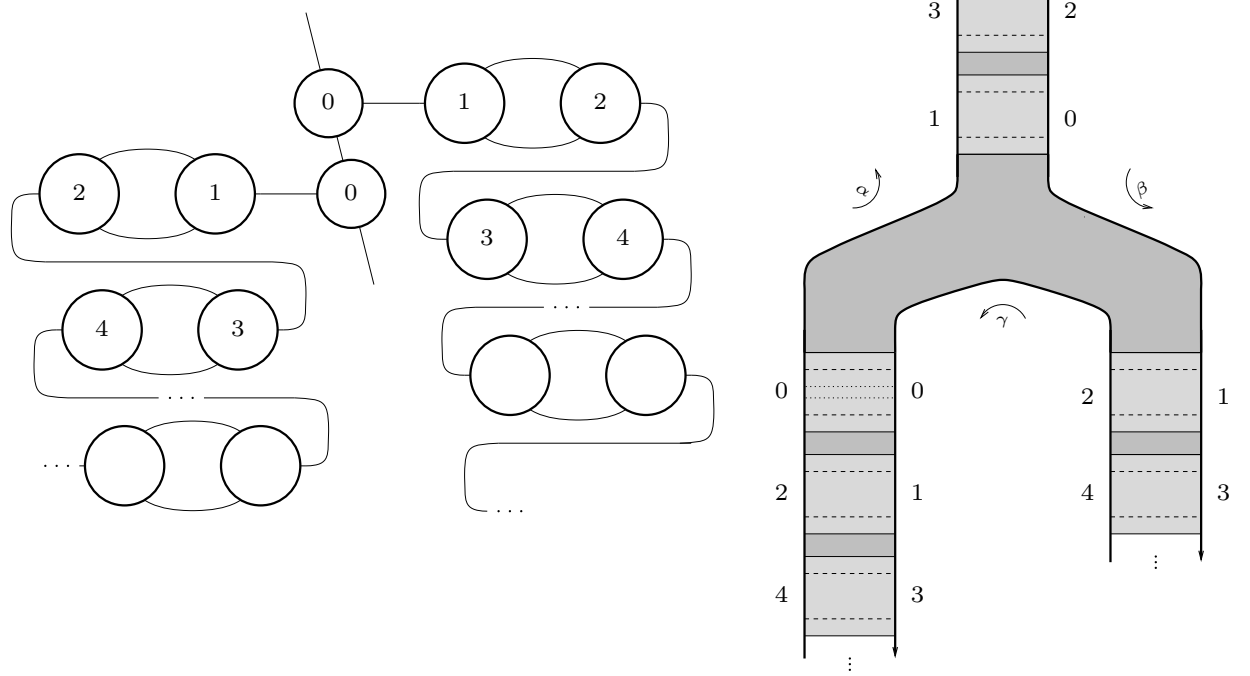

Figure 38 


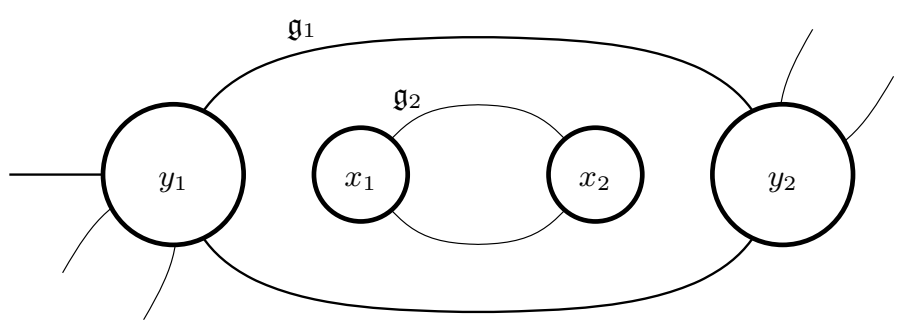

FiguRE 39

\section{Observe:}

- Each branch terminates in a gnarl ( $\sigma$ has been pared down, preserving $c(\sigma)$, so that its third corner is properly contained in $c(\sigma))$.

- Every label in $c(\sigma)$ lies in a unique gnarl or unique trunk (curve of $a(\epsilon)$ ), and in a unique component of $\sigma$. Note that if a label corresponds to the vertex of a trunk curve, then it is in $L(\Sigma)$.

By examining the oscillation of the labels $\{0,1, \ldots\}$ along $c(\sigma)$ in Figure 38 , we get the following.

Property 2. Let $x, y, z$ be vertices on a branch $\mathfrak{B}$ of $\sigma$. Then $x, y$ are of the same sign (as vertices of $\left.G_{F}\right)$ if and only if $b_{\mathfrak{B}}(x) \equiv b_{\mathfrak{B}}(y)(\bmod 2)$. Furthermore, assume $b_{\mathfrak{B}}(x)<b_{\mathfrak{B}}(y)$ and $b_{\mathfrak{B}}(x) \not \equiv b_{\mathfrak{B}}(y)(\bmod 2)$. If either

(a) $b_{\mathfrak{B}}(z)<b_{\mathfrak{B}}(x)$ or

(b) $b_{\mathfrak{B}}(z)<b_{\mathfrak{B}}(y)$ and $b_{\mathfrak{B}}(z) \equiv b_{B}(y)(\bmod 2)$,

then $z \in \overline{x y} \subset c(\sigma)$. In particular, if $x, y$ form a gnarl of $\mathfrak{B}$ and $z$ immediately precedes this gnarl along $\mathfrak{B}$, then $z \in \overline{x y} \subset c(\sigma)$.

Every vertex in $G_{F}$ corresponding to a label in $\sigma$ lies in a unique component of $\sigma$ that includes an essential curve from $a(\epsilon)$, its trunk, and is connected to that curve by edges in its branch. Such a vertex in the interior of a simple gnarl of $\sigma$ must lie on a component of $\sigma$ that, by Property 1, would lie entirely within the interior of the gnarl. In particular, the essential trunk curve of this component lies within the simple gnarl, a contradiction. Thus we have the following:

Property 3. Let $\sigma \in \Sigma_{\mathrm{II}}$. A simple gnarl of $\sigma$ contains no vertices in its interior which are labels of $\sigma$.

Lemma 7.7. Thinking of a branch as a directed path of gnarls in $\widehat{F}$, if a branch of $\Sigma_{\mathrm{II}}$ enters the interior of a simple gnarl of $\Sigma_{\mathrm{II}}$, it never leaves. That is, let $\sigma_{1}, \sigma_{2} \in \Sigma_{\mathrm{II}}$. Let $\mathfrak{g}_{1}$ be a simple gnarl of $\sigma_{1}$ and $\mathfrak{B}_{2}$ be a branch of $\sigma_{2}$. If a vertex (of $G_{F}$ ), $x$, of $\mathfrak{B}_{2}$ lies in the interior of $\mathfrak{g}_{1}$, then at least one of the vertices in $\mathfrak{g}_{1}$ is in $\mathfrak{B}_{2}$. Furthermore, if $y$ is a vertex in both $\mathfrak{g}_{1}$ and $\mathfrak{B}_{2}$, then $b_{\mathfrak{B}_{2}}(y)<b_{\mathfrak{B}_{2}}(x)$.

Proof. The vertex $x$ of $\mathfrak{B}_{2}$ in the interior of $\mathfrak{g}_{1}$ must belong to a simple gnarl $\mathfrak{g}_{2}$ of $\mathfrak{B}_{2}$ (otherwise the vertex belongs to an essential simple closed curve intersecting $\mathfrak{g}_{1}$ at most once). Let $y_{1}, y_{2}$ be the vertices of $\mathfrak{g}_{1}$ and $x_{1}, x_{2}$ the vertices of $\mathfrak{g}_{2}$. See Figure 39. As $\mathfrak{B}_{2}$ (as a directed path in $\widehat{F}$ ) must pass through $\mathfrak{g}_{1}$ to reach $x$ from its trunk, we may take $y_{1}$ so that it is in $\mathfrak{B}_{2}$ such that $b_{\mathfrak{B}_{2}}\left(y_{1}\right) \leq b_{\mathfrak{B}_{2}}\left(x_{i}\right), i=1,2$.

We take $x_{i}$ to be the same sign as $y_{i}$. Possibly $x_{1}=y_{1}$ and $x_{2}=x$ or $x_{2}=y_{2}$ and $x_{1}=x$. 
We assume for contradiction that $y_{2}$ is also in $\mathfrak{B}_{2}$ and that $b_{\mathfrak{B}_{2}}\left(y_{2}\right) \geq b_{\mathfrak{B}_{2}}\left(x_{i}\right), i=$ $1,2\left(x_{1}, x_{2}\right.$ are consecutive along $\left.\mathfrak{B}_{2}\right)$. Note that together $c\left(\sigma_{1}\right) \cup c\left(\sigma_{2}\right)$ does not account for all labels. (Otherwise Lemma 7.8 and Property 3 applied to $\mathfrak{g}_{2}$ implies that $\mathfrak{g}_{2}$ must be the only gnarl of $\mathfrak{B}_{2}$. Corollary 7.9 says that there must be a vertex in the interior of $\mathfrak{g}_{1}$ that does not belong to $\mathfrak{g}_{2}$. By Property 3 , this vertex must belong then to a trunk of $\mathfrak{B}_{2}$. But the trunk gives rise to an essential curve on $\widehat{F}$ intersecting $\mathfrak{g}_{1}$ at most once.) Thus $c\left(\sigma_{1}\right), c\left(\sigma_{2}\right)$ overlap on a label interval. Let $\overline{y_{1}, y_{2}}$ be the interval between $y_{1}, y_{2}$ included in both $c\left(\sigma_{1}\right)$ and $c\left(\sigma_{2}\right)$.

Case (I). $x_{2} \neq y_{2}$.

Applying Property 2(b) to the sequence $y_{1}, x_{2}, y_{2}$ on the branch $\mathfrak{B}_{2}$ (with $z=$ $\left.x_{2}\right), x_{2} \in \overline{y_{1}, y_{2}} \sigma_{2}=\overline{y_{1}, y_{2}}$. But then $x_{2} \in c\left(\sigma_{1}\right)$ and in the interior of $\mathfrak{g}_{1}$, contradicting Property [3.

Case (II). $x_{2}=y_{2}$ and $x_{1}=x$.

Then $x_{1}$ is in the interior of $\mathfrak{g}_{1}$. As $b_{\mathfrak{B}_{2}}\left(x_{1}\right) \equiv b_{\mathfrak{B}_{2}}\left(y_{1}\right)(\bmod 2)$ and $b_{\mathfrak{B}_{2}}\left(y_{1}\right)<$ $b_{\mathfrak{B}_{2}}\left(x_{1}\right)$, there must be another vertex $x^{\prime}$ in $\mathfrak{B}_{2}$ in the interior of $\mathfrak{g}_{1}$ such that $b_{\mathfrak{B}_{2}}\left(y_{1}\right)<b_{\mathfrak{B}_{2}}\left(x^{\prime}\right)<b_{\mathfrak{B}_{2}}\left(x_{1}\right)<b_{\mathfrak{B}_{2}}\left(y_{2}\right)$ and such that $b_{\mathfrak{B}_{2}}\left(x^{\prime}\right) \equiv b_{\mathfrak{B}_{2}}\left(y_{2}\right)(\bmod 2)$. Applying Property 2(b) to the sequence $y_{1}, x^{\prime}, y_{2}$ we conclude that $x^{\prime} \in \overline{y_{1}, y_{2}}$. That is, $x^{\prime}$ is a label in $\sigma_{1}$, contradicting Property 3.

By taking a union of faces in the lower arms of Figure 36, we get:

Property 4. Let $\sigma \in \Sigma_{\mathrm{II}}$. Two gnarls in $\sigma$ cobound an embedded annulus constructed from the faces of $\sigma$.

Lemma 7.8. Let $\sigma \in \Sigma_{\mathrm{II}}$. If any simple gnarl of $\sigma$ contains no vertices of $G_{F}$ in its interior, then this gnarl is the only gnarl of $\sigma$ (i.e., all other labels in $\sigma$ are parts of trunks of components of $\sigma)$. There are at most $F_{2}(g)$ such gnarls.

Proof. The proof of this lemma is divided into two cases according to whether or not the extended Scharlemann cycle within $\sigma$ is actually just a Scharlemann cycle.

Case (I). The extended Scharlemann cycle within $\sigma$ is a Scharlemann cycle. Let $e$ be the bigon bounded by this Scharlemann cycle. See Figure 40 for the labeling we will use. The initial gnarl is the gnarl on vertices $j-2, j+1$. We assume that $\sigma$ has more than the initial gnarl, in particular, that there is a face $f_{3}$ as pictured.

Assume some simple gnarl of $\sigma$ bounds a disk $D^{\prime}$ in $\widehat{F}$ whose interior is disjoint from $K$. Let $B$ be the annulus between this gnarl and the initial gnarl given by Property 4. (If the initial gnarl bounds $D^{\prime}$, then $B=\emptyset$.) Set $D=B \cup D^{\prime}$. Then $D$ is a disk bounded by the initial gnarl and can be taken to have interior disjoint from both $K$ and the faces of $\sigma$.

Let $R_{\alpha \gamma}$ be the rectangle on $\partial X$ bounded by the curves $j-2, j, \alpha \cap\left(e \cup f_{1}\right)$ and $\gamma \cap\left(f_{2} \cup e\right)$ that is disjoint from $\beta$. Let $R_{\beta \gamma}$ be the rectangle on $\partial X$ bounded by the curves $j-1, j+1, \beta \cap\left(f_{2} \cup f_{3}\right)$ and $\gamma \cap\left(e \cup f_{1}\right)$ that is disjoint from $\alpha$. (By the curves $j-2, j-1, j, j+1$ on $\partial X$, we mean the curves that are the boundaries of those vertices.) In fact, let $e, e^{\prime}$ be two push-offs of the face $e$ so that $e$ and $f_{2}$ agree along their $(j-1, j)$-edge and so that $e^{\prime}$ agrees with $f_{1}$ along its $(j-1, j)$-edge. Modify $R_{\alpha \gamma}, R_{\beta \gamma}$ along $\partial X$ between $j-1, j$ by deforming it slightly. See Figure 41, 

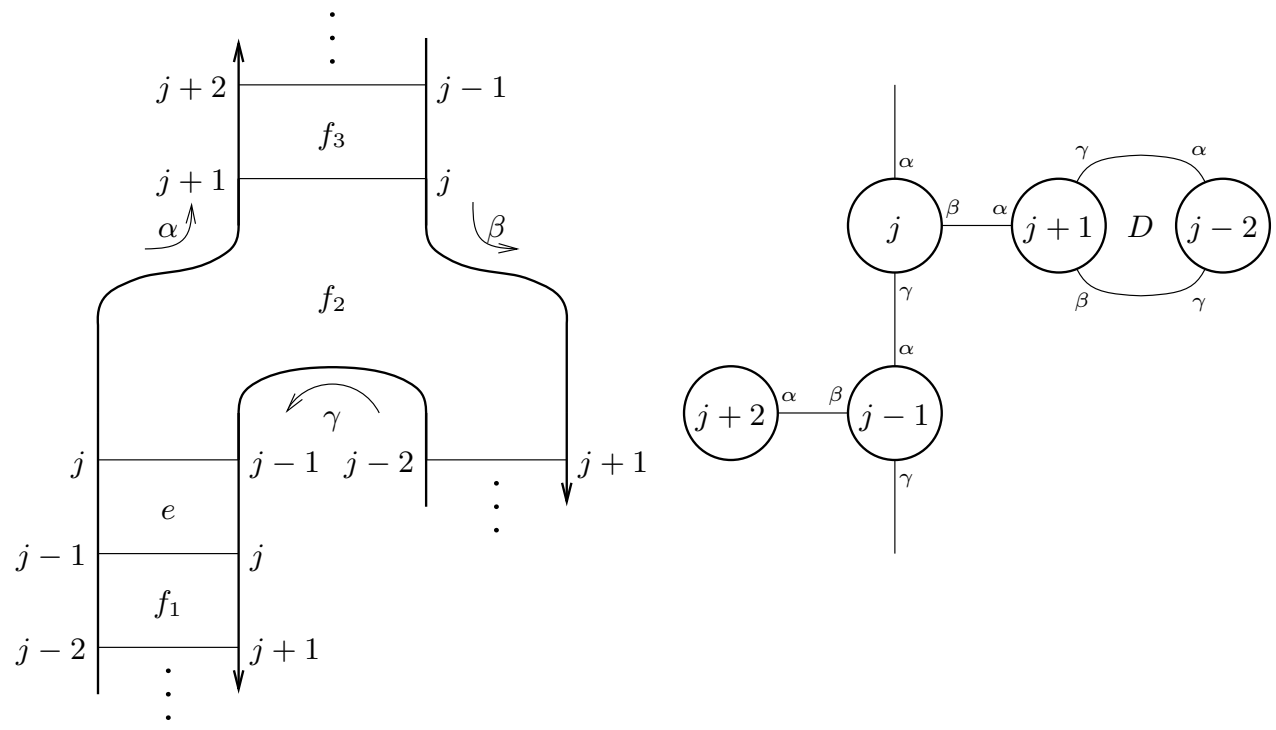

FigURE 40

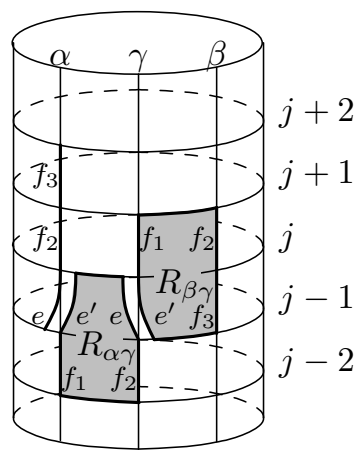

FiguRE 41

Form the disk $e \cup f_{2} \cup f_{3} \cup R_{\alpha \gamma} \cup R_{\beta \gamma} \cup D \cup f_{1} \cup e^{\prime}$ of Figure 42. This gives a thinning long disk $D_{\overline{j-1, j+2}}$ taking the subarc $\overline{j-1, j+2}$ of $K$ (view $\overline{j-1, j+2}$ as part of $\alpha \cap \partial X)$ to the arc on $\widehat{F}$ pictured in Figure 43. This contradicts the thinness of $K$ and finishes the proof of Case (I).

Case (II). The extended Scharlemann cycle in $\sigma$ is not a Scharlemann cycle (has length at least 2).

See Figure 44, We assume that $\sigma$ has more than one gnarl, giving rise to face $f_{4}$ in the figure. Let $R_{\beta \gamma}$ and $R_{\alpha \gamma}$ be the rectangles on $\partial X$ bounded by $j-1 \cup j+$ $1 \cup\left(\left(e \cup f_{4}\right) \cap \beta\right) \cup\left(\left(f_{1} \cup f_{2}\right) \cap \gamma\right)$ and $\ell-1 \cup \ell+1 \cup\left(\left(e \cup f_{3}\right) \cap \gamma\right) \cup\left(\left(f_{1} \cup f_{2}\right) \cap \alpha\right)$, respectively. See Figure 45. As in Case (I), the assumption of the empty simple gnarl allows us to construct a disk $D$ in $X$ which is disjoint from the faces of $\sigma$ and from $K$. Construct the thinning disk $D_{\overline{j-1, j+2}}=e \cup f_{1} \cup f_{3} \cup f_{4} \cup R_{\alpha \gamma} \cup R_{\beta \gamma}$ depicted in Figure 46, $D_{\overline{j-1, j+2}}$ gives an isotopy of $\overline{j-1, j+2}$ onto the arc in $\widehat{F}$ 


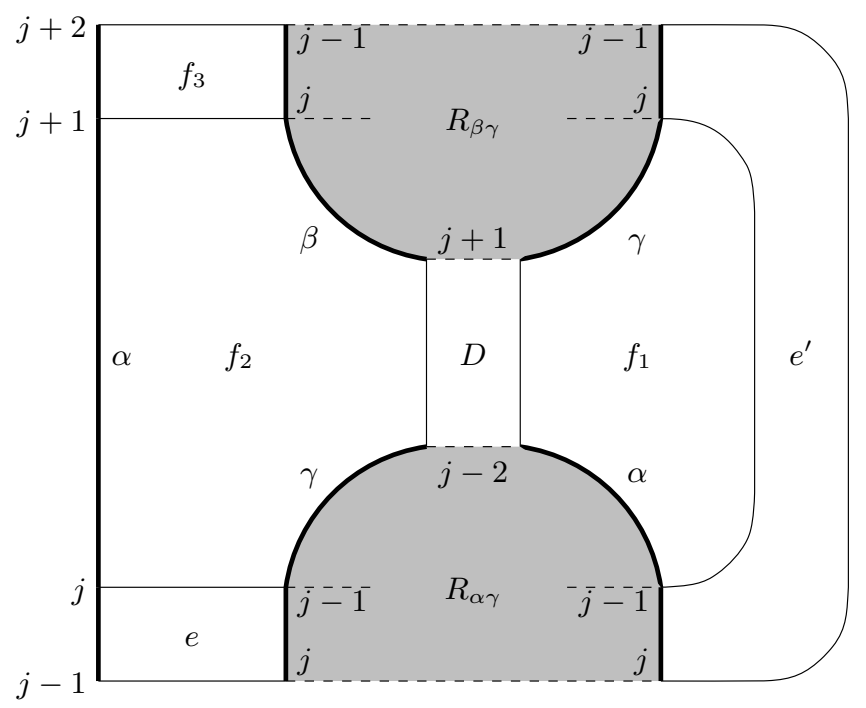

FIGURE 42

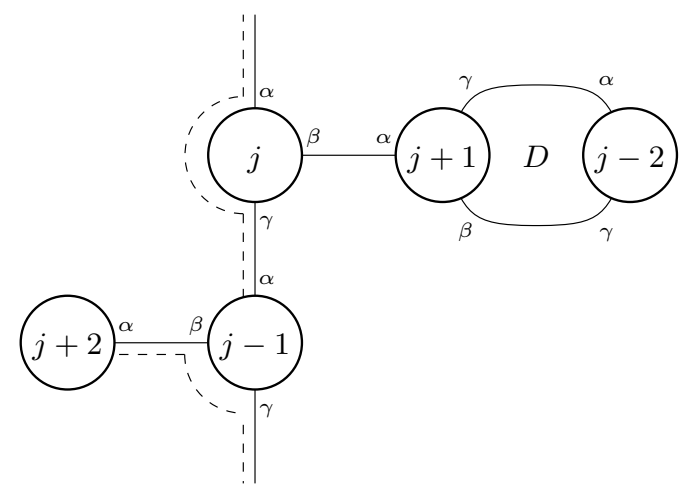

FIGURE 43

indicated in Figure 46, thereby thinning $K$. This contradicts the thinness of $K$ and finishes the proof of Case (II).

For the last statement in the lemma, recall from Remark $7.3(3)$ that $\left|\Sigma_{\mathbb{I I}}\right| \leq$ $F_{2}(g)$. By the above, the gnarls containing no interior vertices belong to different elements of $\Sigma_{\mathrm{mI}}$.

Corollary 7.9. Let $\sigma \in \Sigma_{\mathrm{II}}$. If a gnarl in $\sigma$ has vertices of $G_{F}$ in its interior, it must have at least three. In particular, if $\sigma$ has more than one gnarl, then any simple gnarl of $\sigma$ must contain at least three vertices in the interior of the disk it bounds on $\widehat{F}$.

Proof. If a simple gnarl only has one vertex in its interior, then the graph within would have a 1-sided face (a contradiction), because the number of labels on the interior of the gnarl is less than $q t$ (see Figure 37 for the labeling around a gnarl), 


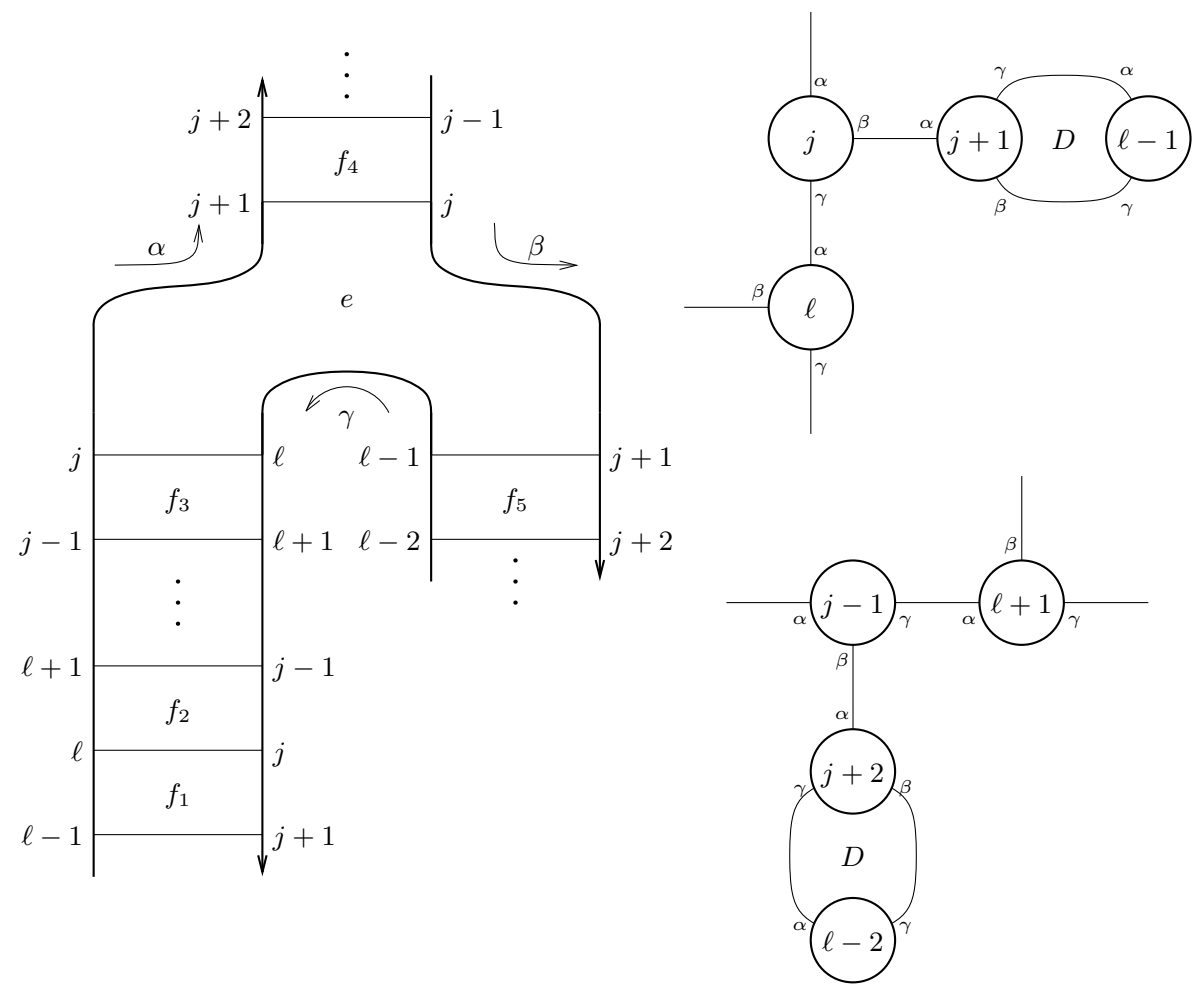

Figure 44

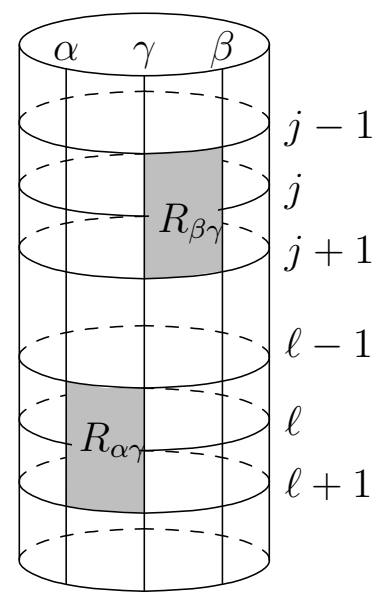

FiguRE 45 


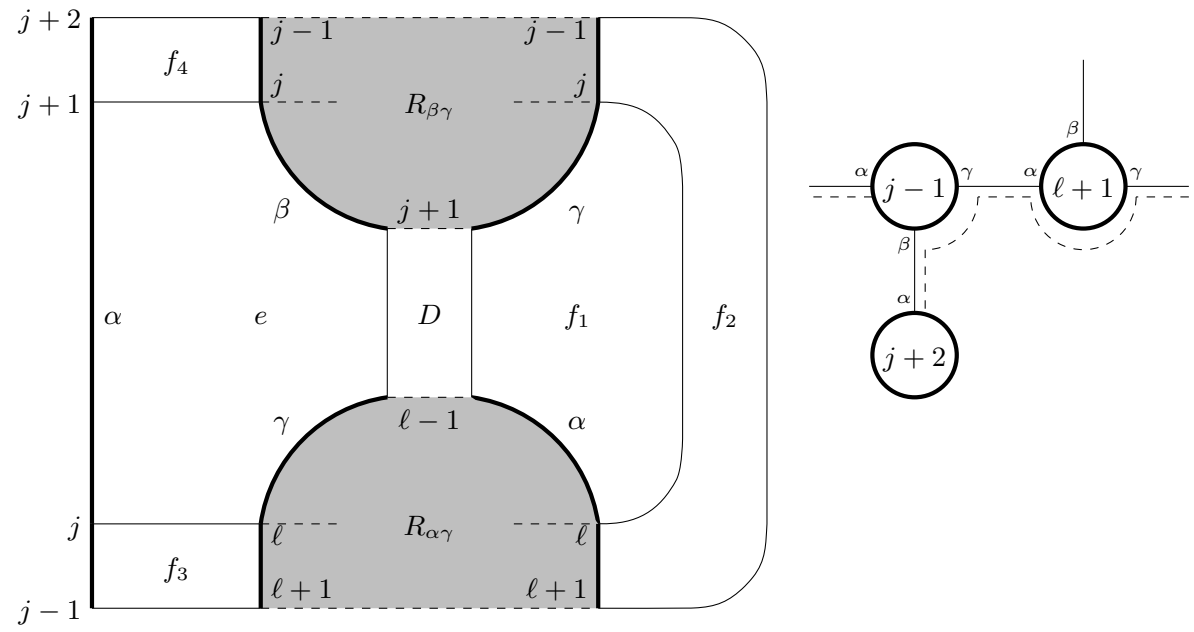

FiguRE 46

the total number of labels (recall that $q$ is the denominator of the Dehn surgery coefficient).

Now assume the simple gnarl on vertices $x, y$ has exactly two vertices $v_{1}$ and $v_{2}$ in its interior. There are fewer than $t$ edges connecting $v_{1}, v_{2}$. Otherwise they are all parallel and describe a cable space in the exterior of $K$ (section 5 of [13]), contradicting that $K$ is hyperbolic. Thus there are more than $2 q t-2 t$ labels on $x, y$ that are endpoints of edges connecting $v_{1}, v_{2}$ to $x, y$ (no 1-sided faces). But there are fewer than $q t$ such labels on $x, y$ in the interior of the gnarl. This is a contradiction since $q$ is at least 2 .

By Lemma 7.8, if $\sigma$ has more than one gnarl, any of its simple gnarls must contain vertices in its interior.

Lemma 7.10. Let $\sigma \in \Sigma_{\mathrm{II}}$. Let $\mathfrak{B}$ be a branch of (a component of) $\sigma$. Let $\mathfrak{t}$ be the trunk of $\mathfrak{B}$ and $\mathfrak{g}$ be a gnarl of $\mathfrak{B}$. If $\mathfrak{t}$ and $\mathfrak{g}$ cobound an annulus $A$ on $\widehat{F}$, then either $\mathfrak{B}$ is disjoint from Int $A$ or $M$ is a Seifert fibered space over the 2-sphere with three exceptional fibers, one having order 2 and another order 3 . In this latter case, if $\widehat{F}$ is from a genus 2 splitting of $M$ with respect to which $K$ has smallest bridge number, then $|K \cap \operatorname{Int} A| \geq t / 2-2$.

Proof. Let $A$ be the annulus in $\widehat{F}$ cobounded by $\mathfrak{g}$ and $\mathfrak{t}$.

If $\sigma$ contains a simple gnarl $\mathfrak{g}_{s}$, then let $D_{s}$ be the disk in $\widehat{F}$ that $\mathfrak{g}_{s}$ bounds. Property 4 gives an annulus $B$ from $\mathfrak{g}_{s}$ to $\mathfrak{g}$ (and transverse to $\widehat{F}$ there) arising from $\sigma$. Therefore, $\mathfrak{g}$ bounds the embedded disk $D=B \cup D_{s} \subset M$ transverse to $\widehat{F}$ along $\mathfrak{g}$ (by Property $3, B$ is disjoint from the interior of $D_{s}$ ). On the other hand, $\mathfrak{t}$ bounds a long Möbius band, $E$, coming from the extended Scharlemann cycle of $\sigma$ which is transverse to $\widehat{F}$ at $\mathfrak{t}$. Then $A \cup D \cup E$ is an immersed projective plane that can be surgered to produce an embedded projective plane in $M$ - a contradiction. Therefore $\sigma$ contains no simple gnarls.

We assume $\mathfrak{B}$ intersects $\operatorname{Int} A$. Then Property 1 along with the fact that $\mathfrak{B}$ contains no simple gnarls guarantees that $\mathfrak{g}$ is the only gnarl in $\mathfrak{B}$. That is, $\mathfrak{B}$ 

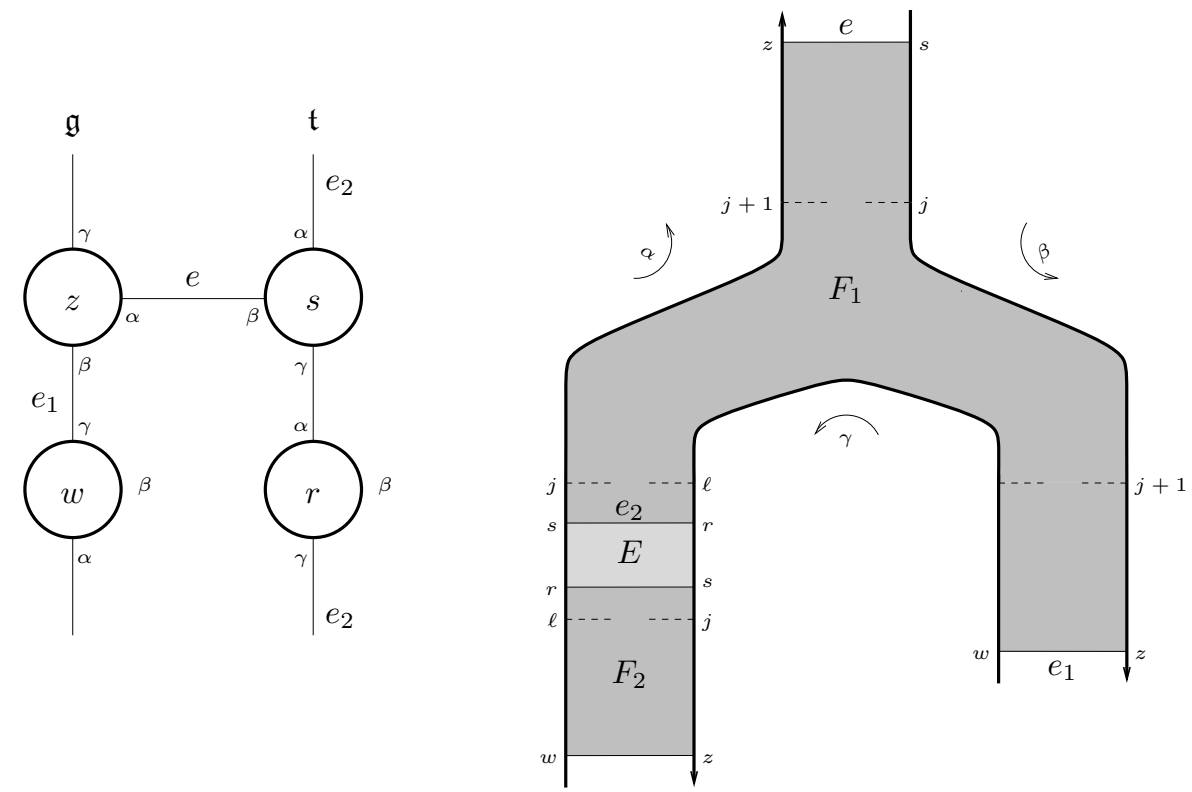

FiguRE 47

consists of $\mathfrak{t}, \mathfrak{g}$, and a single edge $e$ connecting them that lies in $A$. Without loss of generality, we may assume we are as in Figure 47. Note that Int $A$ contains no vertices that appear as labels in $\sigma$, as any such would be part of an essential curve in Int $A$ disjoint from $e$. Similarly, $e$ is the only edge of $\sigma$ contained in $\operatorname{Int} A$.

Let $h_{w r}, h_{r s}$, and $h_{s z}$ be the consecutive handles in $\operatorname{Nbhd}(K)$ running between vertices $w, r, s, z$. Let $N=\operatorname{Nbhd}\left(A \cup h_{s z} \cup h_{w r} \cup F_{1} \cup F_{2}\right)$, where the $F_{i}$ are the disks in $\sigma$ from Figure 47. Then $\pi_{1}(N)$ has presentation $\left\langle x, y, c \mid x^{2} y, x c y c\right\rangle$, where $x, y$ correspond to $h_{s z}, h_{w r}$, respectively, $e, e_{1}, e_{2}$ are retracted to a base point, and $c$ represents the core of $A$. This can be rewritten $\left\langle x, m \mid m^{2} x^{-3}\right\rangle$ (using $m=x c$ ). Thus $N$ is a trefoil knot exterior, and furthermore the core of $A$ is its meridian (attaching a disk along $c$ kills the group). Let $\hat{E}=\operatorname{Nbhd}\left(E \cup h_{r s}\right)$ be a thickening of the long Möbius band coming from the extended Scharlemann cycle, and consider the submanifold $N^{\prime}=N \cup \hat{E}$. As $M$ is atoroidal, $\partial N^{\prime}$ must compress in $M-N^{\prime}$. As $M$ is irreducible, compressing $\partial N^{\prime}$ gives a 2-sphere which bounds a 3-ball in $M$. If this 3 -ball contained $N^{\prime}$, then $M$ would contain a projective plane constructed from a disk in the 3-ball bounded by the meridian of $N$ and the long Möbius band. Thus the 3-ball is disjoint from $N^{\prime}$, and $M-N^{\prime}$ is a solid torus. This implies that $M$ is a Seifert fibered space over the 2 -sphere with three exceptional fibers, one of which has order 2 and another of order 3 .

So we now assume that $\widehat{F}$ is a genus 2 splitting with respect to which $K$ has the smallest bridge number. Let $n=|\operatorname{Int} A \cap K|$. Let $D$ be the disk in $A$ gotten by removing from $A$ an open neighborhood of $\partial A \cup e$. Then $K$ can be perturbed so that it intersects $N^{\prime}$ in $n+2$ arcs, each of the form $p \times I$ in $D \times I$ (e.g. perturb $K$ from label $w$ to label $z$ between $\alpha$ and $\gamma$ in $\operatorname{Nbhd}(K))$.

Now $H^{\prime}=\operatorname{Nbhd}\left(h_{s z} \cup h_{w r} \cup F_{1} \cup F_{2}\right)$ is a genus 2 handlebody as neighborhoods of an arc in $F_{1}$ and an arc in $F_{2}$ give a pair of meridians. Furthermore, the first of 


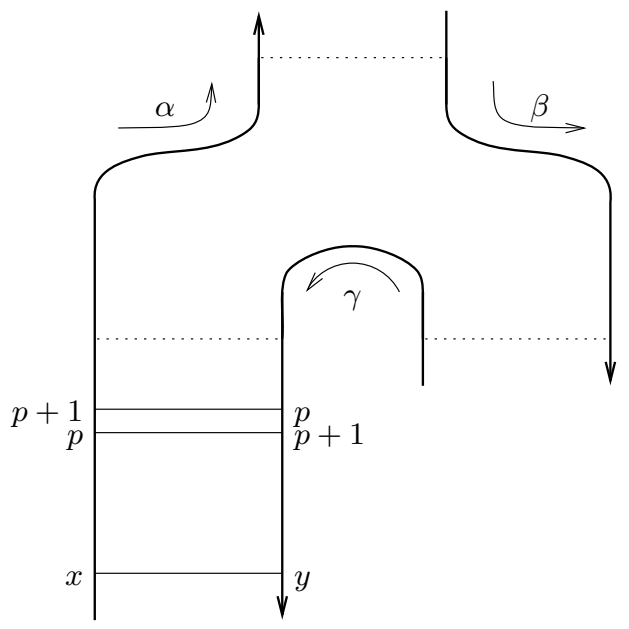

FigURE 48

these meridians shows that the annulus along which $H^{\prime}$ and $\hat{E}$ meet is primitive in $H^{\prime}$. Thus $H=N^{\prime}-D \times I$ is a genus 2 handlebody.

Note that the surgery curve $p / q$ is not a boundary slope for $X$, the exterior of $K$. For as $M$ contains no incompressible surface, this would contradict Theorem 2.0.3 of [7]. Consider the punctured torus $T=\partial N^{\prime}-\operatorname{Nbhd} K$. Maximally compress $T$ in $X$. Then $T$ compresses to $\partial$-parallel annuli. Each $\partial$-parallel annulus defines an isotopy in $M$ of a subarc of $K$, with endpoints in $K \cap \partial N^{\prime}$, onto $\partial N^{\prime}$ and keeping the endpoints of the arc fixed. Furthermore, every point of $K \cap \partial N^{\prime}$ belongs to such an $\operatorname{arc}$ of $K$. Let $k_{1}^{\prime}, \ldots, k_{r}^{\prime}$ be the subarcs of $K$ lying within the outermost of these $\partial$-parallel annuli and let $k_{1}, \ldots, k_{r}$ be the complementary subarcs of $K$. Note that the $k_{i}$ lie on the same side of $\partial N^{\prime}$. Then the isotopies described above corresponding to the outermost annuli deform $K$ to the union of the $\operatorname{arcs} k_{1}, \ldots, k_{r}$ along with arcs that lie in $\partial N$. As the complement of $K$ in $M$ is hyperbolic, it must be that the $\operatorname{arcs} k_{1}, \ldots, k_{r}$ lie in $N^{\prime}$. That is, $k_{1}, \ldots, k_{r}$ are of the form $p \times I$ for $p \in D$. Let $\tau$ be an arc $q \times I$ for $q \in D$ that is disjoint from $K$. Then $\left[N^{\prime}-\operatorname{Nbhd}(\tau)\right] \cup\left[\left(M-N^{\prime}\right) \cup \operatorname{Nbhd}(\tau)\right]$ is a genus 2 Heegaard splitting of $M$. Furthermore, each of $k_{1}, \ldots, k_{r}$ is $\partial$-parallel in the handlebody $N^{\prime}-\operatorname{Nbhd}(\tau)$. Perturbing each arc of $K-\left(\bigcup k_{i}\right)$ into $M-N^{\prime}$ puts $K$ in bridge position with respect to this genus 2 splitting. Noting that $r \leq n+2$ and that $t$ is at most twice the minimal bridge number that $K$ has with respect to a genus 2 splitting of $M$, we get that $t / 2 \leq n+2$, as desired.

Lemma 7.11. A gnarl or trunk of $\Sigma_{\mathrm{II}}$ shares at most a single vertex (of $G_{F}$ ) with another gnarl or trunk of $\Sigma_{\mathrm{II}}$. Furthermore, a vertex of $G_{F}$ belongs to at most two elements among the collection of gnarls and trunks of $\Sigma_{\mathrm{II}}$.

Proof. Let $x, y$ be shared vertices of one gnarl or trunk with another gnarl or trunk. The two must belong to different trigons $\sigma_{1}, \sigma_{2}$ in $\Sigma_{\mathrm{II}}$. Let $p, p+1$ be the labels of the core Scharlemann cycle of one of these trigons. See Figure 48. Then $x+y \equiv 2 p+1$ $(\bmod t)$, implying that $p=(x+y-1) / 2$ or $p=(x+y-1+t) / 2$. If the cores of $\sigma_{1}$ and $\sigma_{2}$ are different, then all labels of $G_{Q}$ are contained in $c\left(\sigma_{1}\right) \cup c\left(\sigma_{2}\right)$ and the 
core Scharlemann cycles are 'antipodal'. By the minimality of $\Sigma_{\mathrm{II}}, \sigma_{1}, \sigma_{2}$ are the only elements of $\Sigma_{\mathrm{II}}$. Because their cores are antipodal, we may pare one of them down so that their corners are disjoint but their union contains all labels. We may then have assumed that this was $\Sigma_{\mathrm{m}}$ and the resulting gnarls and trunks share no labels at all.

If a vertex of $G_{F}$ belongs to three different gnarls or trunks of $\Sigma_{\mathrm{II}}$, then it must belong to $c\left(\sigma_{1}\right) \cap c\left(\sigma_{2}\right) \cap c\left(\sigma_{3}\right)$ for three different trigons in $\Sigma_{\mathrm{II}}$. But this contradicts the minimality of $\Sigma_{\mathrm{II}}$.

Definition 7.12. Lemma 7.11 says that two simple gnarls of $\Sigma_{\mathrm{III}}$ whose interiors overlap must be nested. Thus we define a simple gnarl of $\Sigma_{\mathrm{III}}$ to have depth 0 if it is innermost among simple gnarls of $\Sigma_{\mathrm{II}}$. A simple gnarl of $\Sigma_{\mathrm{II}}$ has depth $n+1$ if it contains a gnarl of depth $n$ in its interior (and no higher).

Lemma 7.13. Any two simple gnarls of $\Sigma_{\mathrm{II}}$ whose interiors overlap (hence are nested) must share a vertex. A simple gnarl of $\Sigma_{\mathrm{II}}$ has at most two gnarls of $\Sigma_{\mathrm{II}}$ nested within it. Every simple gnarl of $\Sigma_{\mathrm{II}}$ has depth at most 1.

Proof. Let $\mathfrak{g}_{1}, \mathfrak{g}_{0}$ be simple gnarls on branches $\mathfrak{B}_{1}, \mathfrak{B}_{0}$ of $\sigma_{1}, \sigma_{0}$ in $\Sigma_{\mathrm{II}}$. Assume $\mathfrak{g}_{0}$ is nested within $\mathfrak{g}_{1}$. By Property $3, \sigma_{1}$ and $\sigma_{0}$ must be different elements of $\Sigma_{\mathrm{II}}$. Let $x_{i}, y_{i}$ be the vertices of the gnarl $\mathfrak{g}_{i}$ for $i=0,1$. Assume that $\left\{x_{0}, y_{0}\right\} \cap\left\{x_{1}, y_{1}\right\}=\emptyset$. As the trunk curve of $\mathfrak{g}_{0}$ is essential in $\widehat{F}$, at least one of $\left\{x_{1}, y_{1}\right\}$ must precede $\mathfrak{g}_{0}$ on $\mathfrak{B}_{0}$, say $x_{1}$. By Property $2(\mathrm{~b}), x_{1} \in \bar{x}_{0}, y_{0} \sigma_{0}$. As Property 3 implies that neither $x_{0}$ nor $y_{0}$ can be in $\bar{x}_{1}, y_{1} \sigma_{1}$, it must be that $y_{1} \in \bar{x}_{0}, y_{0} \sigma_{0}$. By assumption, and the minimality of $\Sigma_{\mathrm{II}}, \bar{x}_{1}, y_{1} \sigma_{1} \subset \bar{x}_{0}, y_{0} \sigma_{2}$. But this contradicts the minimality of $\Sigma_{\text {III }}$. This proves that two nested simple gnarls of $\Sigma_{\text {III }}$ must share a vertex. As a vertex can belong to at most two gnarls in $\Sigma_{\mathrm{II}}$, this implies that a simple gnarl has at most two nested within it.

Let $\mathfrak{g}_{2}, \mathfrak{g}_{1}, \mathfrak{g}_{0}$ be simple gnarls of $\Sigma_{\mathrm{III}}$ with $g_{0}$ nested within $\mathfrak{g}_{1}$ which is nested with $\mathfrak{g}_{2}$. Let $\left\{x_{i}, y_{i}\right\}$ be the vertices of $\mathfrak{g}_{i}, i=0,1,2$. By the paragraph above we may assume $x_{1}=x_{0}$. By Lemma 7.11, $y_{0}$ must lie strictly in the interior of $\mathfrak{g}_{1}$. Again by the paragraph above $\mathfrak{g}_{0}$ and $\mathfrak{g}_{2}$ must share a vertex. It cannot be $y_{0}$, as it lies in the interior of $\mathfrak{g}_{0}$. Thus $x_{0}$ lies in all three gnarls, contradicting Lemma 7.11. Thus a simple gnarl of $\Sigma_{\mathrm{III}}$ has depth at most 1 .

Lemma 7.14. There are at most $6 F_{2}(g)$ wrapping gnarls in $\Sigma_{\mathrm{II}}$ unless $M$ is a Seifert fibered space over the 2-sphere with three exceptional fibers, one having order 2 and another order 3 . In the latter case, if $\hat{F}$ is a genus 2 Heegaard splitting of $M$ with respect to which $K$ has smallest bridge number (among genus 2 splittings), then there are at most $7 F_{2}(g)$ wrapping gnarls in $\Sigma_{\mathrm{II}}$.

Proof. First assume that $M$ is not a Seifert fibered space as described. Let $\mathcal{C}$ be the collection of wrapping gnarls coming from the trigons in $\Sigma_{\mathrm{II}}$, and assume for contradiction that $|\mathcal{C}|>6 F_{2}(g)$. Lemma 7.11 shows that $\mathcal{C}$ satisfies properties $(1),(3),(4)$ of Definition 3.1. If two elements of $\mathcal{C}$ intersect non-transversely, perturb them so they are disjoint so that property (2) of Definition 3.1 is also satisfied. Then Lemma 3.2 guarantees that there are at least seven elements of $\mathcal{C}$ that are isotopic on $\widehat{F}$. Any two such elements must be disjoint. Let $\mathfrak{g}_{1}, \mathfrak{g}_{2}$ be the outermost gnarls of these seven cobounding an annulus $A$. Let $\mathfrak{g}$ be a gnarl in $A$ between $\mathfrak{g}_{1}, \mathfrak{g}_{2}$. Then $\mathfrak{g}$ belongs to a branch of $\Sigma_{\mathrm{II}}$. Call the part of the branch between $\mathfrak{g}$ and its trunk, the short branch of $\mathfrak{g}$. If the short branch of $\mathfrak{g}$ is disjoint from $\mathfrak{g}_{1}, \mathfrak{g}_{2}$, then 
the trunk of $\mathfrak{g}$ is parallel to $\mathfrak{g}$ in $A$; furthermore, as a branch always locally lies on the same side of a constituent gnarl (Property 1), the entire branch will lie in $A$ between $\mathfrak{g}$ and its trunk, contradicting Lemma 7.10. Thus the short branch of $\mathfrak{g}$ must intersect either $\mathfrak{g}_{1}$ or $\mathfrak{g}_{2}$. As each vertex of a branch either belongs to a gnarl or trunk curve, we may associate to $\mathfrak{g}, b(\mathfrak{g})$, the first gnarl or trunk curve on the short branch that shares a vertex with either $\mathfrak{g}_{1}$ or $\mathfrak{g}_{2}$ (orienting the short branch from $\mathfrak{g}$ to its trunk). If $\mathfrak{g}, \mathfrak{g}^{\prime}$ are different gnarls between $\mathfrak{g}_{1}, \mathfrak{g}_{2}$, then $b(\mathfrak{g})$ and $b\left(\mathfrak{g}^{\prime}\right)$ must be different. Otherwise, $\mathfrak{g}, \mathfrak{g}^{\prime}$ belong to the same branch $\left(b(\mathfrak{g})=b\left(\mathfrak{g}^{\prime}\right)\right.$ cannot be the trunk curve of two different branches by Lemma 7.11) and are therefore disjoint before perturbation. Then $\mathfrak{g}^{\prime}$, say, must occur on the short branch of $\mathfrak{g}$. As above, Property 1 shows that the entire branch of $\mathfrak{g}$ lies between $\mathfrak{g}, \mathfrak{g}^{\prime}$, contradicting Lemma 7.10. Similarly, one sees that $b(\mathfrak{g})$ is neither $\mathfrak{g}_{1}$ nor $\mathfrak{g}_{2}$. Lemma 7.11 says that there are at most four trunks or gnarls of $\Sigma_{\mathrm{II}}$ that share a vertex with $\mathfrak{g}_{1}$ or $\mathfrak{g}_{2}$, other than $\mathfrak{g}_{1}$ or $\mathfrak{g}_{2}$ themselves. This contradicts that there are five gnarls between $\mathfrak{g}_{1}, \mathfrak{g}_{2}$.

Now assume $M$ is a Seifert fibered space over the 2 -sphere with three exceptional fibers including orders 2,3 and $\widehat{F}$ comes from a genus 2 splitting for which $K$ has the smallest bridge number. Let $\mathcal{C}$ be the collection of wrapping gnarls coming from the trigons in $\Sigma_{\mathrm{II}}$, and assume for contradiction that $|\mathcal{C}|>7 F_{2}(g)$. Now at least eight elements of $\mathcal{C}$ are isotopic on $\widehat{F}$. The above shows that there must be two gnarls $\mathfrak{g}, \mathfrak{g}^{\prime}$ of $\mathcal{C}$ whose branches lie entirely in an annulus $A$ on $\widehat{F}$. In particular, the trunk curves of these gnarls are parallel (or equal) in $A$. Then by Corollary [5.15] and Remark 7.3(3), there is a $\sigma \in \Sigma_{\mathrm{II}}$ containing both of these branches. These branches then are either disjoint or share the same trunk curve. Let $A_{1}, A_{2}$ be the sub-annuli of $A$ that lie between these gnarls and their trunk curves. Then the interiors of these annuli are disjoint and each intersects the branch of the corresponding gnarl. Lemma 7.10 shows that $t-4$ vertices of $G_{F}$ lie in the union of the interiors of these annuli. But there are at least six more vertices coming from the gnarls and their trunk curve(s), contradicting that $G_{F}$ has $t$ vertices.

\section{Proof of Theorem 8.1}

In this section we prove our main theorem, which is the following.

Theorem 8.1. There is a linear function $w: \mathbb{N} \rightarrow \mathbb{N}$ with the following property. Let $K^{\prime}$ be a hyperbolic knot in $S^{3}, M=K^{\prime}(p / q)$ where $q \geq 2$, and $K$ the core of the attached solid torus in $M$. Suppose $K$ is in thin position with respect to a genus $g$ Heegaard splitting of $M$ and let $S$ be a corresponding thick level surface. If $S$ is a strongly irreducible Heegaard surface for $M$, then either

(1) $|K \cap S| \leq 2 w(g)$; or

(2) $M$ is toroidal; or

(3) $M$ is a Seifert fibered space over the 2-sphere with exactly three exceptional fibers, at least one of which has order 2 or 3.

Furthermore, in cases (2) and (3) $M$ has a genus 2 Heegaard splitting with respect to which $K$ has bridge number 0 in case (2) and at most $w(2)$ in case (3).

Theorem 8.1 is proved by establishing the existence of a collection of annuli in $S=\widehat{F}$ whose boundary components are, roughly speaking, elements of $a \theta(\Sigma)$, and which capture, in their interiors, vertices of $G_{F}$ that do not correspond to labels in $\mathcal{L}$. This is stated precisely in Lemma 8.3, which is proved in subsection 8.1 
below. Before giving the proof of the lemma, we show how it leads to the proof of Theorem 8.1

Proof of Theorem 8.1. Let $K^{\prime}$ be a knot in $S^{3}$ and $M=K^{\prime}(p / q)$ where $q \geq 2$. Let $K$ be the core of the attached solid torus in $K^{\prime}(p / q)$. We are given a genus $g$, strongly irreducible Heegaard surface $S$ for $M$, and we may assume $K$ cannot be isotoped onto this surface, else we may take (1) to hold. In the special case that $M$ is a Seifert fibered space as in (3), we further assume that $S$ comes from a genus 2 Heegaard splitting of $M$ with respect to which $K$ has the smallest bridge number among all genus 2 splittings of $M$. Note that as $M$ is neither a lens space, $S^{3}$, $S^{1} \times S^{2}$, nor a connected sum, any genus 2 splitting of $M$ is strongly irreducible. Then there are surfaces $F, Q$ in the exterior of $K^{\prime}$ as described by Lemma 2.3 , where $\widehat{F}=S$ is a thick level surface in a thin presentation of $K$ with respect to the given splitting. Let $G_{F}, G_{Q}$ be the corresponding graphs of intersection. Let $t=|\partial F|=|K \cap \widehat{F}|$. We assume that $M$ is atoroidal so that (2) of Theorem 8.1 does not hold. We then show that either conclusion $(1)($ for $w(g)=10,581(g-1)+394)$ or (3) (with the bridge number bound on the minimal bridge number, genus 2 splitting) of Theorem 8.1 holds.

We may assume $t \geq 2 g-2$, so Lemma 2.5 guarantees that $G_{Q}$ has a great web $\Lambda$. Let $\mathcal{L}$ be the collection of labels of $\Lambda$ given in Definition 2.7, and $-\mathcal{L}$ the complement of $\mathcal{L}$ among all labels of $G_{Q}$. Let $\Sigma$ be the minimal collection of extended Scharlemann cycles of $\Lambda$ given by Definition 4.9 , Let $\Sigma_{\mathrm{II}}$ and $\Sigma_{\mathrm{II}}$ be as in Definitions 6.7 and 7.2. Then for every $x \in \mathcal{L}$, either $x \in L(\Sigma)$ or there is a $\sigma \in \Sigma_{\mathrm{II}} \cup \Sigma_{\mathrm{III}}$ such that $x \in c(\sigma)$. Let $\mathfrak{G}$ be the set of labels of $G_{Q}$ that correspond to vertices of simple gnarls of $\Sigma_{\mathrm{II}}$ that are not in $L(\Sigma)$ (section 7 ). A gnarl hereafter will always be a gnarl of $\Sigma_{\mathrm{II}}$.

Every element of $\mathcal{L}$ belongs to either $L(\Sigma), \mathcal{L}_{\mathrm{II}}$ (Definition 6.6), or corresponds to a vertex of a gnarl (Definition (7.6). A gnarl may be either wrapping or simple. By Theorem 6.24 and Lemma 7.14

$$
|\mathcal{L}| \leq|L(\Sigma)|+|\mathfrak{G}|+960(g-1)+14 F_{2}(g) .
$$

Let $\mathcal{A}$ be the collection of annuli given by Lemma 8.3. We can write $\mathfrak{G}=\mathfrak{G}^{\prime} \amalg \mathfrak{G}^{\prime \prime}$, where $\mathfrak{G}^{\prime}$ are the elements of $\mathfrak{G}$ corresponding to vertices that lie outside $\bigcup_{B \in \mathcal{A}} B$, and $\mathfrak{G}^{\prime \prime}$ are those corresponding to vertices in the interiors of the annuli in $\mathcal{A}$.

Using Lemma 7.8 to remove from consideration those gnarls with no vertices in their interior, Corollary 7.9 and Lemma 7.13 imply that there are at least $\left|\mathfrak{G}^{\prime}\right|-$ $2 F_{2}(g)$ vertices of $G_{F}$ outside $\bigcup_{B \in \mathcal{A}} B$ that lie in the interior of simple gnarls but are not vertices of gnarls. Such vertices cannot belong to $L(\Sigma)$, so they are either in $\mathcal{L}_{\text {II }}$ or in $-\mathcal{L}$.

Claim 8.2. There are at least $\left|\mathfrak{G}^{\prime}\right|-2 F_{2}(g)$ vertices of $G_{F}$ that lie outside of $\bigcup_{B \in \mathcal{A}} B$ and that correspond to elements of $-\mathcal{L} \cup \mathcal{L}_{\mathbb{I I}}$.

Proof. Let $\overline{\mathfrak{G}^{\prime}}$ be the set of elements of $\mathfrak{G}^{\prime}$ that do not belong to simple gnarls that contain no vertices in their interior. By Lemma $\left[7.8,\left|\overline{\mathfrak{G}^{\prime}}\right| \geq\left|\mathfrak{G}^{\prime}\right|-2 F_{2}(g)\right.$. To the elements of $\overline{\mathfrak{G}^{\prime}}$ we associate distinct elements of $-\mathcal{L} \cup \mathcal{L}_{\mathrm{II}}$ that lie outside $\bigcup_{B \in \mathcal{A}} B$.

Consider a depth 1 gnarl $\mathfrak{g}$ that contains an element of $\overline{\mathfrak{G}^{\prime}}$ (as one of its vertices). By Corollary 7.9 and Lemma 7.13 , there are at least as many vertices in the interior of $\mathfrak{g}$ that do not belong to gnarls as there are elements of $\overline{\mathfrak{G}^{\prime}}$ that lie in $\mathfrak{g}$ or its interior ( $\mathfrak{g}$ contains at most two depth 0 gnarls, each of which shares a vertex with 
$\mathfrak{g}$, and two such gnarls have disjoint interiors). These elements in the interior of $\mathfrak{g}$ that do not belong to gnarls cannot be in $L(\Sigma)$, hence must correspond to elements of $-\mathcal{L} \cup \mathcal{L}_{\mathbb{I I}}$. Furthermore, as they are interior to $\mathfrak{g}$, they must lie outside of $\bigcup_{B \in \mathcal{A}} B$. To the elements of $\overline{\mathfrak{G}^{\prime}}$ in $\mathfrak{g}$ or its interior we associate distinct elements of $-\mathcal{L} \cup \mathcal{L}_{\mathbb{I}}$ among these.

In sequence, consider all elements of $\overline{\mathfrak{G}^{\prime}}$ that lie in depth 1 gnarls that have not previously been assigned an element of $-\mathcal{L} \cup \mathcal{L}_{\mathbb{I I}}$. Apply the above procedure to assign elements of $-\mathcal{L} \cup \mathcal{L}_{\mathrm{II}}$ to that element of $\overline{\mathfrak{G}^{\prime}}$ along with any others that lie in that gnarl or its interior.

Each of the elements of $\overline{\mathfrak{G}^{\prime}}$ not assigned elements of $-\mathcal{L} \cup \mathcal{L}_{\mathrm{II}}$ by the above process must lie in a depth 0 gnarl (with interior vertices) that does not itself lie in a depth 1 gnarl containing an element of $\overline{\mathfrak{G}^{\prime}}$. Consider such a depth 0 gnarl. By Corollary 7.9, we may associate distinct vertices in the interior of $\mathfrak{g}$ to the elements of $\overline{\mathfrak{G}^{\prime}}$ that belong to $\mathfrak{g}$. These interior vertices will again belong to $-\mathcal{L} \cup \mathcal{L}_{\mathbb{I}}$ and lie outside of $\bigcup_{B \in \mathcal{A}} B$. By looking at such depth 0 gnarls we may thus sequentially assign elements of $-\mathcal{L} \cup \mathcal{L}_{\mathrm{II}}$ to the remaining elements of $\overline{\mathfrak{G}^{\prime}}$.

In the above procedure, no two elements of $\overline{\mathfrak{G}^{\prime}}$ can be assigned the same element of $-\mathcal{L} \cup \mathcal{L}_{\mathbb{I}}$, for each such element of $-\mathcal{L} \cup \mathcal{L}_{\mathbb{I}}$ comes from the interior of a certain gnarl that contains an element of $\overline{\mathfrak{G}^{\prime}}$. By Lemma 7.13, if two gnarls share an interior vertex, one must be nested in the other. So one associated gnarl must be depth 1 , the other depth 0 . This is prohibited by the above procedure.

First assume $\mathcal{A}$ is non-empty. By Claim 8.2 and part (5) of Lemma 8.3 ,

$$
\begin{aligned}
|-\mathcal{L}|+\left|\mathcal{L}_{\mathrm{II}}\right| & \geq\left|\mathfrak{G}^{\prime}\right|-2 F_{2}(g)+(3 / 7) \sum_{B \in \mathcal{A}}\left(n_{B}+4\right) \\
& =\left|\mathfrak{G}^{\prime}\right|+(3 / 7)\left|\mathfrak{G}^{\prime \prime}\right|+(3 / 7) 4|\mathcal{A}|-2 F_{2}(g) \\
& \geq(3 / 7)(|\mathfrak{G}|+|L(\Sigma)|-4 l(g))-2 F_{2}(g) \\
& \geq(3 / 7)\left(|\mathcal{L}|-960(g-1)-14 F_{2}(g)-4 l(g)\right)-2 F_{2}(g),
\end{aligned}
$$

where $l(g)$ is the function defined in Lemma 8.3 , and where the last line uses $(*)$. Thus by Theorem 6.24

$$
|-\mathcal{L}| \geq(3 / 7)\left(|\mathcal{L}|-960(g-1)-14 F_{2}(g)-4 l(g)\right)-2 F_{2}(g)-960(g-1) .
$$

By Proposition 2.9, $|\mathcal{L}| \geq(3 / 4) t-(g-1) / 2$. Therefore

$$
\begin{aligned}
t= & |-\mathcal{L}|+|\mathcal{L}| \\
\geq & (1+3 / 7)((3 / 4) t-(g-1) / 2)-(3 / 7)\left(960(g-1)+14 F_{2}(g)+4 l(g)\right) \\
& -2 F_{2}(g)-960(g-1) \\
= & (15 / 14) t-(5 / 7)(g-1)-(3 / 7)\left(960(g-1)+14 F_{2}(g)+4 l(g)\right) \\
& -2 F_{2}(g)-960(g-1) .
\end{aligned}
$$

Thus $t \leq 2 w(g)$, where

$$
\begin{aligned}
2 w(g) & =10(g-1)+6 \cdot 960(g-1)+24 l(g)+84 F_{2}(g)+28 F_{2}(g)+14 \cdot 960(g-1) \\
& =19,210(g-1)+24 l(g)+112 F_{2}(g) \\
& =19,210(g-1)+24(58(g-1)+47 / 2)+112(5(g-1)+2) \\
& =21,162(g-1)+788,
\end{aligned}
$$

proving Theorem 8.1 when $\mathcal{A}$ is non-empty. 
Now assume $\mathcal{A}$ is empty. Then Lemma 8.3(1) implies $|L(\Sigma)| \leq 4 l(g)$. Furthermore, $\mathfrak{G}=\mathfrak{G}^{\prime}$, and Claim 8.2 along with Theorem 6.24 yields $|-\mathcal{L}| \geq$ $|\mathfrak{G}|-2 F_{2}(g)-960(g-1)$ (this is the analog of $\left.(* *)\right)$. Then $(*)$ gives $|-\mathcal{L}| \geq$ $|\mathcal{L}|-1920(g-1)-16 F_{2}(g)-4 l(g)$. Using Proposition 2.9, we get

$$
\begin{aligned}
t & =|-\mathcal{L}|+|\mathcal{L}| \\
& \geq(3 / 2) t-1921(g-1)-16 F_{2}(g)-4 l(g) .
\end{aligned}
$$

This gives $t \leq 2\left(1921(g-1)+16 F_{2}(g)+4 l(g)\right)<2 w(g)$, where $w(g)$ is as above.

8.1. The collection of annuli, $\mathcal{A}$. If $\theta$ is a $\theta$-curve, a curve in $\theta$ is a circle $\gamma$ obtained by removing from $\theta$ the interior of an edge. If $\sigma$ is an extended Scharlemann cycle of length $3, \gamma(\sigma)$ will denote the set of curves in $\theta$-curves belonging to $\theta(\sigma)$. If $\sigma$ is an extended Scharlemann cycle of length 2 or $3, a \gamma(\sigma)$ will denote $a(\sigma) \cup \gamma(\sigma)$ (where $\gamma(\sigma)=\emptyset$ if $\sigma$ has length 2).

Lemma 8.3. There is a (possibly empty) collection $\mathcal{A}$ of annuli in $\widehat{F}$ such that

(1) $|\mathcal{A}| \geq|L(\Sigma)| / 4-l(g)$, where $l(g)=58(g-1)+47 / 2$;

(2) for any $B \in \mathcal{A}$ there is a $\sigma \in \Sigma$ such that each component of $\partial B$ belongs to $a \gamma(\sigma)$;

(3) the interiors of any two distinct annuli in $\mathcal{A}$ are disjoint;

(4) no vertex in the interior of an annulus in $\mathcal{A}$ belongs to $L(\Sigma)$, and any vertex in the interior of an annulus in $\mathcal{A}$ that belongs to $\mathcal{L}$ is either a vertex of a simple gnarl or belongs to $\mathcal{L}_{\mathrm{II}}$;

(5) if $B \in \mathcal{A}$ and $n_{B}$ is the number of vertices in the interior of $B$ that belong to simple gnarls, then there are at least $(3 / 7)\left(n_{B}+4\right)$ vertices in $B$ that belong to $-\mathcal{L} \cup \mathcal{L}_{\mathbb{I}}$.

Remark 8.4. If $\mathcal{A}$ is empty $(|\mathcal{A}|=0)$, then (1) says that $|L(\Sigma)| \leq 4 l(g)$.

Proof. By Lemma 4.15 there are at least $|\theta(\Sigma)|-3 F_{2}(g) \theta$-curves of $\theta(\Sigma)$ that lie in essential annuli. Since $|L(\Sigma)| / 2 \leq|a(\Sigma)|+|\theta(\Sigma)|$ and each element of $a(\Sigma)$ lies in an essential annulus, at least $|L(\Sigma)| / 2-3 F_{2}(g)$ of the elements of $a \theta(\Sigma)$ lie in essential annuli. Let $\mathcal{C}$ be this subcollection of $a \theta(\Sigma)$. Call two elements of $\mathcal{C}$ isotopic if the cores of the corresponding annuli are isotopic in $\widehat{F}$. Then any two elements of $\mathcal{C}$ which are isotopic are either disjoint or intersect non-transversely in a single vertex. Furthermore, the elements of $\mathcal{C}$ form at most $F_{2}(g)$ isotopy classes (using 'core' curves from the $\theta$-curves and perturbing non-transverse intersections, Lemma 4.12 shows that a subcollection of non-isotopic elements of $\mathcal{C}$ have property $P(2)$ - apply Lemma 3.2). Thus all but at most $3 F_{2}(g)$ of these curves lie in isotopy classes with at least four elements of $\mathcal{C}$. Let $\mathcal{C}_{1}, \ldots, \mathcal{C}_{m}$ be the distinct isotopy classes of $\mathcal{C}$, each of which contains at least four elements of $\mathcal{C}$.

A pinched annulus $A \subset \widehat{F}$ is a disk $D$ with two points on its boundary identified to a single point $v$. The interior of $A$ is the interior of $D$. Note that under the identification $\partial D$ becomes the union of the two simple closed curves $\gamma$ and $\gamma^{\prime}$ that intersect non-transversely at $v$. We write $\partial A=\gamma \cup \gamma^{\prime}$.

Consider $\mathcal{C}_{i}=\left\{c_{1}, c_{2}, \ldots, c_{n}\right\}$, say, where $c_{r} \in a \theta(\Sigma), 1 \leq r \leq n$, and the $c_{r}$ 's are ordered sequentially as they lie on $\widehat{F}$. Then we have

(R1) $c_{r}$ and $c_{s}$ are disjoint unless $|r-s|=1$ or $\{r, s\}=\{1, n\}$, and $c_{r}$ and $c_{s}$ share a single vertex (Lemma 4.12); 
(R2) for $1 \leq r<n$ there exists $A_{r} \subset \widehat{F}$ such that

(a) $A_{r}$ is an annulus or pinched annulus according to whether $c_{r} \cap c_{r+1}$ is empty or non-empty;

(b) $\partial A_{r}=\gamma_{r} \cup \gamma_{r+1}$, where $\gamma_{s}$ is a curve in $c_{s}, s=r, r+1$;

(c) $A_{r}$ contains $c_{r} \cup c_{r+1}$;

(d) there are no vertices of any $a \theta(\Sigma)$ in the interior of $A_{r}$.

We have $c_{r} \in a \theta\left(\sigma_{r}\right), \sigma_{r} \in \Sigma$.

Note that if $A_{r}$ is pinched, then $\sigma_{r} \neq \sigma_{r+1}$.

Claim 8.5. If $\sigma_{r} \neq \sigma_{r+1}$, then the number of vertices of $G_{F}$ in the interior of $A_{r}$ is at least $t / 2-3$, and at least $t / 2-2$ if $A_{r}$ is pinched.

Proof. We may assume $t>32(g-1)$, hence Lemmas 5.12 and 5.16 imply that $M$ is a Seifert fibered space over $S^{2}$ with three exceptional fibers at least one of which has order 2 or 3 . This puts us in the context of Theorem 8.1 (3). Thus $\widehat{F}$ is a genus 2 splitting surface for $M$ with respect to which $K$ has the smallest bridge number. Again by Lemmas 5.12 and 5.16, $K$ has bridge number at most $k-1$, where $k=\left|K \cap A_{r}\right|$. Thus $t / 2 \leq k-1$ (twice the bridge number is an upper bound for the number of intersections with a thick surface). Let $k^{\prime}$ be the number of vertices in the interior of $A_{r}$. Then $k=k^{\prime}+4$ or $k^{\prime}+3$ according to whether $A_{r}$ is an annulus or a pinched annulus, giving $k^{\prime} \geq t / 2-3$ or $t / 2-2$, respectively.

Claim 8.6. If the elements of $a \theta(\Sigma)$ are incident to at most $v$ vertices of $G_{F}$, then $|a \theta(\Sigma)| \leq v$. In particular, we may assume $v>2 l(g)=116(g-1)+47$.

Proof. Each vertex of $G_{F}$ belongs to at most two different elements of $a \theta(\Sigma)$ by Lemma 4.12. On the other hand, every element of $a \theta(\Sigma)$ involves two vertices of $G_{F}$. Thus $v \geq|a \theta(\Sigma)|=|a(\Sigma)|+|\theta(\Sigma)| \geq|L(\Sigma)| / 2$. If $v \leq 2 l(g)$, then $4 l(g) \geq|L(\Sigma)|$, and taking $\mathcal{A}=\emptyset$ satisfies Lemma 8.3 .

Let $\mathcal{A}_{i}^{+}$be the collection $\left\{A_{r} \mid 1 \leq r<n\right\}$ coming from $\mathcal{C}_{i}$.

Claim 8.7. For any $i \neq j$, any element of $\mathcal{C}_{i}$ is disjoint from some element of $\mathcal{C}_{j}$. Thus any element of $\mathcal{C}_{i}$ is either disjoint from a given element of $\mathcal{C}_{j}$ or intersects it non-transversely in a single vertex. No vertex in the interior of an annulus of $\mathcal{A}_{i}^{+}$ lies in the interior of an annulus of $\mathcal{A}_{j}^{+}, i \neq j$.

Proof. Assume that $\mathcal{A}_{i}^{+}$contains two non-adjacent pinched annuli. Then these annuli have disjoint interiors. By Claim 8.5 there are at least $t-4$ vertices of $G_{F}$ in the interiors of these annuli. Then $(\mathrm{R} 2)(\mathrm{d})$ implies there are at most four vertices of $G_{F}$ belonging to $|a \theta(\Sigma)|$, contradicting Claim 8.6. Thus any pinched annuli in $\mathcal{A}_{i}^{+}$are adjacent and there are at most two pinched annuli in $\mathcal{A}_{i}^{+}$. Since $\left|\mathcal{C}_{i}\right| \geq 4$, there are at least three disjoint elements of $\mathcal{C}_{i}$. The middle of these is then disjoint from any element of $\mathcal{C}_{j}$ as proposed, and any element of $\mathcal{C}_{j}$ can intersect an element of $\mathcal{C}_{i}$ only non-transversely. Let $A \in \mathcal{A}_{i}^{+}, B \in \mathcal{A}_{j}^{+}$. By the above, the boundary components of $A, B$ can be perturbed to be disjoint. If their interiors intersected, then some component of $\partial A$ would be isotopic to one of $\partial B$, a contradiction. Thus $A, B$ share no interior vertices.

Let $\mathcal{A}^{+}=\bigcup_{i=1}^{m} \mathcal{A}_{i}^{+}$. It follows from Claims 8.5, 8.6, and 8.7, that there are at most two elements $A \in \mathcal{A}^{+}$such that $\partial A=\gamma \cup \gamma^{\prime}, \gamma \in a \gamma(\sigma), \gamma^{\prime} \in a \gamma\left(\sigma^{\prime}\right)$, where $\sigma \neq \sigma^{\prime}$, and that, if there are two, then they are adjacent (and hence belong to 
the same $\mathcal{A}_{i}^{+}$). Let $\widetilde{\mathcal{A}_{i}^{+}}$be $\mathcal{A}_{i}^{+}$with any such elements removed (only one family is changed). Then for each $i$ there is a $\sigma \in \Sigma$ such that for any annulus $B \in \widetilde{\mathcal{A}_{i}^{+}}$, $\partial B \subset a \gamma(\sigma)$. In particular, there are no pinched annuli in $\widetilde{\mathcal{A}_{i}^{+}}$.

If $A^{+}, B^{+} \in \widetilde{\mathcal{A}_{i}^{+}}$, their interiors will be disjoint unless they share a $\theta$-curve $\theta$, in which case two of the edges of $\theta$ will cobound a disk, $D$, of parallelism in $\widehat{F}$ with $D \subset A^{+} \cap B^{+}$. Since the cores of the annuli in $\widetilde{\mathcal{A}_{i}^{+}}$are all isotopic in $\widehat{F}$, assigning an orientation to this isotopy class allows us to talk about one element of $\widetilde{\mathcal{A}_{i}^{+}}$being to the right or left of another. If $A^{+}, B^{+} \in \widetilde{\mathcal{A}_{i}^{+}}$share a disk of parallelism $D$, with $A^{+}$on the left of $B^{+}$, we define $A=A^{+}-\operatorname{Int} D$. Doing this for all pairs in $\widetilde{\mathcal{A}_{i}^{+}}$that share a disk of parallelism, we get a collection $\widetilde{\mathcal{A}_{i}^{+}}$of annuli in $\widehat{F}$ whose interiors are disjoint.

Let $\tilde{\mathcal{A}}=\bigcup_{i=1}^{m} \widetilde{\mathcal{A}_{i}^{+}}$. Then $\tilde{\mathcal{A}}$ satisfies conditions (2) and (3) of the lemma. Also, recall from the first paragraph of the proof that $|\mathcal{C}| \geq|L(\Sigma)| / 2-3 F_{2}(g)$ and $\sum_{i=1}^{m}\left|\mathcal{C}_{i}\right| \geq|\mathcal{C}|-3 F_{2}(g)$. Hence

$$
\begin{aligned}
|\tilde{\mathcal{A}}|=\sum_{i=1}^{m} \widetilde{\mathcal{A}_{i}^{+}} \mid & \geq \sum_{i=1}^{m}\left(\left|\mathcal{C}_{i}\right|-1\right)-2 \\
& \geq|L(\Sigma)| / 2-m-2-6 F_{2}(g) .
\end{aligned}
$$

Claim 8.8. There are at most $|\tilde{\mathcal{A}}| / 2+(m+1) / 2$ annuli in $\tilde{\mathcal{A}}$ with no vertices of $G_{F}$ in their interior.

Proof. The elements of $\widetilde{\mathcal{A}_{i}^{+}}$appear sequentially along $\widehat{F}$. Since $\tilde{\mathcal{A}}=\bigcup \widetilde{\mathcal{A}_{i}^{+}}$is obtained from $\mathcal{A}^{+}=\bigcup_{i=1}^{m} \mathcal{A}_{i}^{+}$by removing either 0,1 or 2 adjacent elements from some $\mathcal{A}_{i}^{+}$, the annuli in $\tilde{\mathcal{A}}$ appear in at most $(m+1)$ sequential groups, with, say $n_{r}$ annuli in the $r$ th group, $1 \leq r \leq m+1$.

We show that no two consecutive annuli in $\widetilde{\mathcal{A}_{i}^{+}}$have empty interiors. So assume $A, B$ are consecutive annuli in $\widetilde{\mathcal{A}_{i}^{+}}$, neither of which contains vertices in its interior. By (R2) and the construction of $\widetilde{\mathcal{A}_{i}^{+}}, \partial A$ and $\partial B$ belong to $a \gamma(\sigma)$ for some $\sigma \in \Sigma$. But this contradicts Lemma 5.9 or Lemma 5.10 (or Theorem 8.1 (3) holds with the bridge number bound). Note that if $\sigma$ were of length 3, letting $\theta \in a \theta(\sigma)$ be the $\theta$-curve which meets both $A$ and $B$, then, by the way we defined the elements of $\tilde{\mathcal{A}}$, the disk of parallelism of $\theta$ is contained in either $A$ or $B$. So Lemma 5.10 applies.

It follows that the number of annuli in $\tilde{\mathcal{A}}$ with no vertices in their interior is at most

$$
\sum_{r=1}^{m+1}\left(\frac{n_{r}+1}{2}\right)=\frac{|\tilde{\mathcal{A}}|}{2}+\frac{(m+1)}{2}
$$

as claimed.

Since any label in $L(\Sigma)$ corresponds to a vertex in an element of $a \theta(\Sigma)$, it is clear from the definition of the original collection $\mathcal{A}^{+}$that no annulus in $\tilde{\mathcal{A}}$ contains vertices in its interior corresponding to elements of $L(\Sigma)$. 
Finally, we define $\mathcal{A}$ to be the collection of annuli obtained by discarding from $\tilde{\mathcal{A}}$ any annulus whose interior either

- has no vertices of $G_{F}$; or

- contains the vertex of a wrapping gnarl; or

- contains the vertex of a simple gnarl with no vertices in its interior.

Then $\mathcal{A}$ satisfies condition (2), (3), and (4) of Lemma 8.3 .

By Claim 8.8, Lemma 7.14, and Lemma 7.8,

$$
\begin{aligned}
|\mathcal{A}| & \geq|\tilde{\mathcal{A}}|-(|\tilde{\mathcal{A}}|+(m+1)) / 2-7 F_{2}(g)-F_{2}(g) \\
& =(|\tilde{\mathcal{A}}|-(m+1)) / 2-8 F_{2}(g) .
\end{aligned}
$$

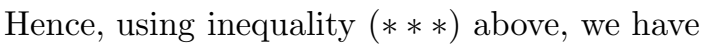

$$
|\mathcal{A}| \geq|L(\Sigma)| / 4-m-3 / 2-11 F_{2}(g) .
$$

By Claim 8.7 and Lemma 3.2, $m \leq F_{0}(g)=3 g-3$. Setting $F_{2}(g)=5(g-1)+2$, we get

$$
|\mathcal{A}| \geq|L(\Sigma)| / 4-l(g),
$$

where $l(g)=58(g-1)+47 / 2$. Thus $\mathcal{A}$ satisfies condition (1).

It remains to show that $\mathcal{A}$ satisfies condition (5).

Claim 8.9. Each element of $\mathcal{A}$ contains at least two vertices in its interior.

Proof. Assume the annulus $B \in \mathcal{A}$ contains a single vertex in its interior. By (2), the components of $\partial B$ belong to $a \gamma(\sigma)$ for the same element, $\sigma$, of $\Sigma$.

First assume that $\sigma$ is an extended Scharlemann cycle of length 2. Then there is an annulus $A \subset A(\sigma)$ that runs between the components of $\partial B$. $A$ intersects $B$ only along their mutual boundary, forming a 2-torus, $T$. Furthermore, $K$ may be perturbed off $A$ so that it intersects $T$ either once or three times. But then $T$ is a non-separating surface in $M$, which is impossible.

When $\sigma$ has length 3 , we arrive at an analogous contradiction. $B$ contains a $\theta$-curve $\theta \in \theta(\sigma)$. As in the proof of Corollary [7.9, if the unique vertex of $G_{F}$ in the interior of $B$ were to lay between the two parallel edges of $\theta$ in $B$, then $G_{F}$ would have a 1-sided face (the number of edges interior to the parallelism is less than the valence of the interior vertex). Hence this vertex cannot lie in this disk of parallelism. The proof of Lemma 5.3 shows how to construct an annulus $A$ within $\Theta(\sigma)$ that runs between components of $\partial B$ such that $K$ can be perturbed off of $A$ to intersect $B$ transversely in either one or three points. But then $A \cup B$ is a non-separating torus in $M$, a contradiction.

Let $B \in \mathcal{A}$, let $n_{B}$ be the number of vertices in the interior of $B$ that belong to simple gnarls, and let $\overline{n_{B}}$ the number that correspond to labels in $-\mathcal{L} \cup \mathcal{L}_{\mathrm{II}}$. We must show that $\overline{n_{B}} \geq(3 / 7)\left(n_{B}+4\right)$. Note that if a vertex in the interior of $B$ belongs to $\mathcal{L}$, it must in fact belong to $\mathcal{L}_{\mathrm{II}}$ by condition $(4)$ of Lemma 8.3 .

If $n_{B}=0$, then $\overline{n_{B}} \geq 2>(3 / 7) 4$ by Claim 8.9 .

If $n_{B}>0$, then the corresponding vertices belong to simple gnarls where each such gnarl contains vertices in its interior. By Lemma 7.13, simple gnarls are of depth 0 or 1. By Corollary [7.9, each depth 0 gnarl contains in its interior at least 
three vertices, which must belong to $-\mathcal{L} \cup \mathcal{L}_{\mathrm{II}}$ and hence contribute 3 to $\overline{n_{B}}$. By Lemma 7.13, a depth 1 gnarl must share a vertex with an internal depth 0 gnarl. By Lemma 7.11, a vertex of $G_{F}$ belongs to at most two different gnarls.

Let $n_{B}^{\prime}$ be the vertices that belong to gnarls lying in $B$ (possibly with vertices on $\partial B$ ). Then $n_{B} \leq n_{B}^{\prime}$. Let $n$ be number of depth 0 gnarls contained in $B, k$ be the number of vertices in depth 1 gnarls in $B$ that are not in depth 0 gnarls, and $r$ the number of vertices that are contained in two depth 0 gnarls in $B$. From the observations in the preceding paragraph, $n_{B}^{\prime}=2 n-r+k$. Furthermore, there are at least $3 n$ vertices in $-\mathcal{L} \cup \mathcal{L}_{\mathrm{II}}$ in the interior of $B$. Thus $\left|-\mathcal{L} \cup \mathcal{L}_{\mathrm{II}}\right| \geq 3 n=$ $n_{B}+\left[\left(n_{B}^{\prime}-n_{B}\right)+r+(n-k)\right]$. Noting that $n \geq k$, the bracketed quantity is non-negative. Therefore,

$$
\overline{n_{B}} \geq \begin{cases}n_{B}, & \text { if } n_{B} \equiv 0(\bmod 3), \\ n_{B}+2, & \text { if } n_{B} \equiv 1(\bmod 3), \\ n_{B}+1, & \text { if } n_{B} \equiv 2(\bmod 3) .\end{cases}
$$

It follows that $\overline{n_{B}} \geq(3 / 7)\left(n_{B}+4\right)$.

\section{Appendix, INTERSECTIONS With INCOMPRESSIBLE SURFACES}

Theorem 9.1. There is a linear function $w_{I}: \mathbb{N} \rightarrow \mathbb{N}$ with the following property. Let $K^{\prime}$ be a hyperbolic knot in $S^{3}, M=K^{\prime}(p / q)$ where $q \geq 2$, and $K$ the core of the attached solid torus in $M$. Let $S$ be an orientable, incompressible surface in $M$ of genus $g$. Then $K$ can be isotoped to intersect $S$ at most $w_{I}(g)$ times.

Remark 9.2. Compare the above with Theorem 4 of 34 which says the following. Let $K^{\prime}$ be a hyperbolic knot in $S^{3}$ that does not contain a genus $g$, closed incompressible surface in its exterior and such that $M=K^{\prime}(p / q)$ contains an incompressible surface of genus $g$. Let $K$ be the core of the attached solid torus in $M$. If $q \geq 4$, then there is an incompressible surface of genus $g$ in $M$ which $K$ intersects at most $\frac{36}{q-3}(g-1)$ times.

Proof. The proof is just that of Theorem 8.1 given for strongly irreducible Heegaard surfaces, except basically that the proof of Claim5.13 must be altered to replace the assumption of strong irreducibility with incompressibility. In fact the arguments for an incompressible surface tend to be easier than in the context of a Heegaard surface, but as those arguments have already been made, we will make use of them. As $M$ is a non-integral surgery on a knot in $S^{3}$, it is irreducible ([14]).

We may assume that $M$ is atoroidal. Otherwise by [17] $K^{\prime}$ is an Eudave-Muñoz knot and, consequently, $M$ is the union of two Seifert fibered spaces over the disk, each with two exceptional fibers, along a torus $S$. The Seifert fibers of the two halves intersect once along $S$. Hence $S$ is the unique orientable incompressible surface. (Any other connected incompressible surface would have to be the union of horizontal surfaces in the two Seifert fibered spaces. Consequently such a surface would be non-separating, contradicting that $M$ is a rational homology sphere.) Again [17] shows that $K$ can be isotoped to intersect $S$ twice. Note that this also shows that $M$ cannot be a Seifert fibered space, as any such which is atoroidal contains no incompressible surface. Finally, recall that $M$ cannot contain a Klein bottle by Theorem 1.3 of [16] (see also [6]). 
So let $\widehat{F}$ be an incompressible surface in $M$ of genus at least 2 and isotop $K$ to intersect $\widehat{F}$ minimally. Let $F$ be the punctured surface, $\widehat{F} \cap X$, where $X$ is the exterior of $K$ in $M$ (which is the exterior of $K^{\prime}$ in $S^{3}$ ). The thin position argument given in Lemma 4.4 of [10] (in place of, and simpler than, that of [26]) shows that there is a level surface $\widehat{Q}$ in a thin presentation of $K^{\prime}$ in $S^{3}$ such that the corresponding punctured surface $Q=\widehat{Q} \cap X$ intersects $F$ in arcs which are essential in both $Q$ and $F$. That is, no arcs of $F \cap Q$ are boundary parallel in either $F$ or $Q$.

The combinatorial arguments of section 2.2 go through in this context. In particular, Proposition 2.9 still holds for $\mathcal{L}$ as defined in that section. For each label $x \in \mathcal{L}$, there is a bigon or trigon in $\Lambda_{x}$, and the classification of such as either an extended Scharlemann cycle or as a trigon of Type I, II, or III is the same.

One checks that all of the results of section 5 hold, however, with the helpful addition now that $M$ cannot be a Seifert fibered space. Note that an isotopy which gave a thinning in this section now reduces the intersection number of $K$ and $\widehat{F}$. Indeed this is overkill. For example, neither conclusion of Lemma 5.5] can hold in this context: conclusion (1) allows us to reduce the intersection of $K$ with $\widehat{F}$, and conclusion (2) says that $M$ is a Seifert fibered space. There is one place in section 5 where the fact that $\widehat{F}$ is a strongly irreducible Heegaard surface is used. That is, in the proof of Claim 5.13. Strong irreducibility is used there to show that the Seifert fibered space $N$ does not lie in a 3-ball. In the current context, this does not occur, as it would imply that a curve that is homotopically essential in $\widehat{F}$ lies in this 3-ball, and consequently is homotopically trivial in $M$ - thereby violating the incompressibity of $\widehat{F}$.

The results of sections 6 and 7 follow from the supporting lemmas in section 5 and the fact that $M$ is irreducible, atoroidal, and contains no Klein bottle.

Finally, section 8 pulls together these supporting lemmas to give the bound $w_{I}(g)=2 w(g)$ on $|K \cap \widehat{F}|$.

\section{ACKNOWLEDGements}

The authors would like to thank the referee for detailed and helpful comments.

\section{REFERENCES}

[1] Kenneth L. Baker, Small genus knots in lens spaces have small bridge number, Algebr. Geom. Topol. 6 (2006), 1519-1621 (electronic), DOI 10.2140/agt.2006.6.1519. MR2253458 (2007f:57008)

[2] Kenneth L. Baker, Cameron McA. Gordon, and John Luecke, Bridge number and integral Dehn surgery, arXiv:1303.7018 [math.GT].

[3] Kenneth L. Baker, Cameron Gordon, and John Luecke, Obtaining genus 2 Heegaard splittings from Dehn surgery, Algebr. Geom. Topol. 13 (2013), no. 5, 2471-2634, DOI 10.2140/agt.2013.13.2471. MR3116298

[4] John Berge, Some knots with surgeries yielding lens space, unpublished manuscript.

[5] Ryan Blair, Marion Campisi, Jesse Johnson, Scott A. Taylor, and Maggy Tomova, Bridge distance, Heegaard genus, and Exceptional Surgeries, arXiv:1209.0197 [math.GT]

[6] S. Boyer and X. Zhang, On Culler-Shalen seminorms and Dehn filling, Ann. of Math. (2) 148 (1998), no. 3, 737-801, DOI 10.2307/121031. MR.1670053(2000d:57028)

[7] Marc Culler, C. McA. Gordon, J. Luecke, and Peter B. Shalen, Dehn surgery on knots, Ann. of Math. (2) 125 (1987), no. 2, 237-300, DOI 10.2307/1971311. MR881270 (88a:57026)

[8] Mario Eudave-Muñoz, Non-hyperbolic manifolds obtained by Dehn surgery on hyperbolic knots, Geometric topology (Athens, GA, 1993), AMS/IP Stud. Adv. Math., vol. 2, Amer. Math. Soc., Providence, RI, 1997, pp. 35-61. MR1470720 (98i:57007) 
[9] Mario Eudave-Muñoz, On hyperbolic knots with Seifert fibered Dehn surgeries, Proceedings of the First Joint Japan-Mexico Meeting in Topology (Morelia, 1999), Topology Appl. 121 (2002), no. 1-2, 119-141, DOI 10.1016/S0166-8641(01)00114-6. MR1903687 (2003c:57005)

[10] David Gabai, Foliations and the topology of 3-manifolds. III, J. Differential Geom. 26 (1987), no. 3, 479-536. MR910018 (89a:57014b)

[11] Hiroshi Goda and Masakazu Teragaito, Dehn surgeries on knots which yield lens spaces and genera of knots, Math. Proc. Cambridge Philos. Soc. 129 (2000), no. 3, 501-515, DOI 10.1017/S0305004100004692. MR1780501 (2001g:57011)

[12] C. McA. Gordon, Combinatorial methods in Dehn surgery, Lectures at KNOTS '96 (Tokyo), Ser. Knots Everything, vol. 15, World Sci. Publ., River Edge, NJ, 1997, pp. 263-290, DOI 10.1142/9789812796097_0010. MR.1474525 (98m:57020)

[13] C. McA. Gordon and R. A. Litherland, Incompressible planar surfaces in 3-manifolds, Topology Appl. 18 (1984), no. 2-3, 121-144, DOI 10.1016/0166-8641(84)90005-1. MR769286 (86e:57013)

[14] C. McA. Gordon and J. Luecke, Only integral Dehn surgeries can yield reducible manifolds, Math. Proc. Cambridge Philos. Soc. 102 (1987), no. 1, 97-101, DOI 10.1017/S0305004100067086. MR886439(89a:57003)

[15] C. McA. Gordon and J. Luecke, Knots are determined by their complements, J. Amer. Math. Soc. 2 (1989), no. 2, 371-415, DOI 10.2307/1990979. MR965210 (90a:57006a)

[16] C. McA. Gordon and J. Luecke, Dehn surgeries on knots creating essential tori. I, Comm. Anal. Geom. 3 (1995), no. 3-4, 597-644. MR1371211 (96k:57003)

[17] C. McA. Gordon and John Luecke, Non-integral toroidal Dehn surgeries, Comm. Anal. Geom. 12 (2004), no. 1-2, 417-485. MR2074884 (2005k:57013)

[18] A. E. Hatcher, On the boundary curves of incompressible surfaces, Pacific J. Math. 99 (1982), no. 2, 373-377. MR658066 (83h:57016)

[19] John Hempel, 3-manifolds as viewed from the curve complex, Topology 40 (2001), no. 3, 631-657, DOI 10.1016/S0040-9383(00)00033-1. MR.1838999(2002f:57044)

[20] J. Johnson, Bridge number and the curve complex, arXiv:math/0603102 [math.GT].

[21] Yair N. Minsky, Yoav Moriah, and Saul Schleimer, High distance knots, Algebr. Geom. Topol. 7 (2007), 1471-1483, DOI 10.2140/agt.2007.7.1471. MR.2366166 (2008k:57016)

[22] Justin Malestein, Igor Rivin, and Louis Theran, Topological designs, Geom. Dedicata 168 (2014), no. 1, 221-233, DOI 10.1007/s10711-012-9827-9. MR 3158040

[23] Yoav Moriah and Hyam Rubinstein, Heegaard structures of negatively curved 3-manifolds, Comm. Anal. Geom. 5 (1997), no. 3, 375-412. MR.1487722 (98j:57029)

[24] John Kirkpatrick Osoinach Jr., Manifolds obtained by Dehn surgery on infinitely many distinct knots in S(3), ProQuest LLC, Ann Arbor, MI, 1998. Thesis (Ph.D.)-The University of Texas at Austin. MR2697691

[25] John K. Osoinach Jr., Manifolds obtained by surgery on an infinite number of knots in $S^{3}$, Topology 45 (2006), no. 4, 725-733, DOI 10.1016/j.top.2006.02.001. MR2236375 (2007m:57010)

[26] Yo'av Rieck, Heegaard structures of manifolds in the Dehn filling space, Topology 39 (2000), no. 3, 619-641, DOI 10.1016/S0040-9383(99)00026-9. MR1746912 (2001b:57037)

[27] Yo'av Rieck and Eric Sedgwick, Finiteness results for Heegaard surfaces in surgered manifolds, Comm. Anal. Geom. 9 (2001), no. 2, 351-367. MR.1846207 (2002j:57040)

[28] Yo'av Rieck and Eric Sedgwick, Persistence of Heegaard structures under Dehn filling, Topology Appl. 109 (2001), no. 1, 41-53, DOI 10.1016/S0166-8641(99)00147-9. MR.1804562 (2001k:57021)

[29] Martin Scharlemann, Heegaard splittings of compact 3-manifolds, Handbook of geometric topology, Eds. R. Daverman and R. Sher, North-Holland, Amsterdam, 2002, pp. 921-953. MR,1886684 (2002m:57027)

[30] Masakazu Teragaito, A Seifert fibered manifold with infinitely many knot-surgery descriptions, Int. Math. Res. Not. IMRN 9 (2007), Art. ID rnm 028, 16, DOI 10.1093/imrn/rnm028. MR2347296(2008g:57008)

[31] Masakazu Teragaito, Toroidal Dehn surgery on hyperbolic knots and hitting number, Topology Appl. 157 (2010), no. 1, 269-273, DOI 10.1016/j.topol.2009.04.037. MR2556104 (2011d:57023)

[32] Abigail Thompson, Thin position and bridge number for knots in the 3-sphere, Topology 36 (1997), no. 2, 505-507, DOI 10.1016/0040-9383(96)00010-9. MR.1415602 (97m:57013) 
[33] Maggy Tomova, Distance of Heegaard splittings of knot complements, Pacific J. Math. 236 (2008), no. 1, 119-138, DOI 10.2140/pjm.2008.236.119. MR2398991(2009b:57017)

[34] Cynthia Janet Verjovsky Marcotte, Essential surfaces after Dehn filling, ProQuest LLC, Ann Arbor, MI, 2000. Thesis (Ph.D.)-The University of Texas at Austin. MR2701527

Department of Mathematics, University of Miami, Coral Gables, Florida 33146

E-mail address: kb@math.miami.edu

Department of Mathematics, The University of Texas at Austin, Austin, Texas 78712

E-mail address: gordon@math.utexas.edu

Department of Mathematics, The University of Texas at Austin, Austin, Texas 78712

E-mail address: luecke@math.utexas.edu 